\title{
CALCULATIONS OF BOTTOM QUARK PRODUCTION AT HADRON COLLIDERS *
}

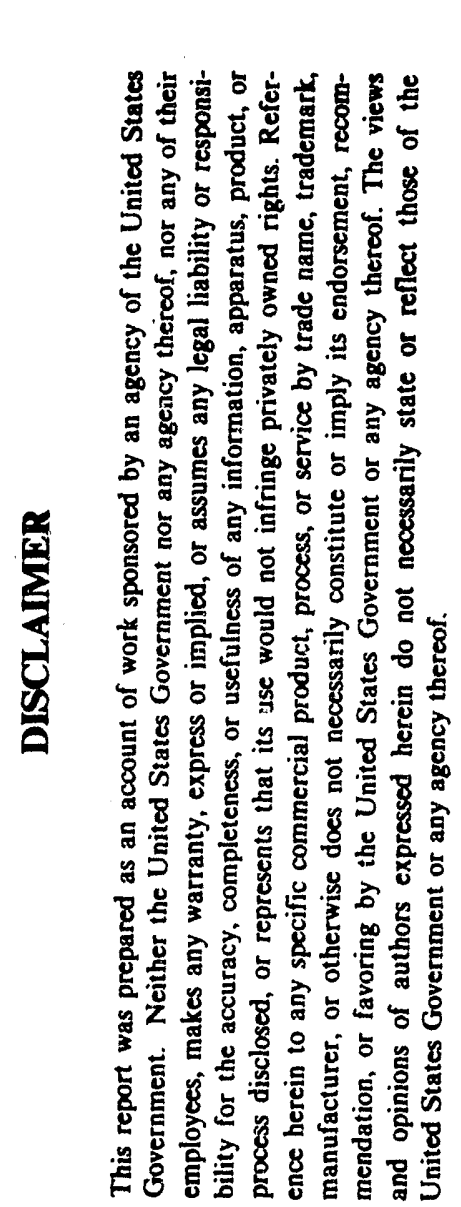

\author{
D. Kuebel
}

Department of Physics

University of Chicago

Chicago, IL 60637

and

High Energy Physics Division

Argonne National Laboratory

9700 South Cass Avenue

Argonne, IL 60439

Ph.D. Thesis submitted to the University of Chicago, 1991.

*Work supported by the U.S. Department of Energy, Division of High Energy Physics, Contract W-31-109-ENG-38. 
ANL-HEP-TR-91-111

June 29, 1991

\title{
CALCULATIONS OF BOTTOM QUARK PRODUCTION AT HADRON COLLIDERS *
}

\author{
D.Kuebel \\ Der,artment of Physics \\ University of Chicago
}

\begin{abstract}
This thesis studies Monte Carlo simulations of QCD heavy flavor production processes $(p \bar{p} \rightarrow Q(\bar{Q}) X)$ at hadron colliders. ISAJET bottom quark cross-sections are compared to the $O\left(\alpha_{s}^{3}\right)$ perturbative calculation of Nason, Dawson, and Ellis. These Monte Carlo cross-sections are computed from data samples which use different parton distribution functions and physics parameters. Distributions are presented in the heavy quark's transverse momentum and rapidity. Correlations in rapidity and azimuthal angle are computed for the heavy flavor pair. Theory issues which arise are the behavior of the crosssection at low and high values of transverse momentum and the treatment of double counting problems in the flavor excitation samples. An important result is that ISAJET overestimates bottom quark production cross-sections and $K$ factors. These findings are relevant for estimates of rates and backgrounds of heavy flavor events.
\end{abstract}

-Work supported by the U.S. Department of Energy, Division of High Energy Physics, Contract W-31-109-ENG-38. 


\section{ACKNOWLEDGMENTS}

The description of the ISAJET samples in chapter 3 and the first half of chapter 4 are essentially extracts from reference 20 . My debts to the coauthors are as follows:

Ed Berger served as supervisor to the project.

Mark Pundurs generated the samples at $630 \mathrm{GeV}$ and prepared figures 13 through 17 . He also designed the layout for table 1.

C.P. Yuan gave me several informal lessons on Monte Carlos and allowed me to use his parton level Monte Carlo to make figures 11 and 12.

Frank Paige came to Argonne in the fall of 1988 and introduced me to ISAJET.

I would also like to thank former University of Chicago experimental students John Conway and Joel Heinrich for handling my computer difficulties at the University. Ruibin Meng discussed some aspects of the higher order calculation with me. Miuch graitude goes to the high energy physics theory group at Argonne for their support and considerable patience. The work at Argonne was supported by the U.S. Department of Energy, Division of High Energy Physics, Contract No. W-31-109ENG-38. 


\section{TABLE OF CONTENTS}

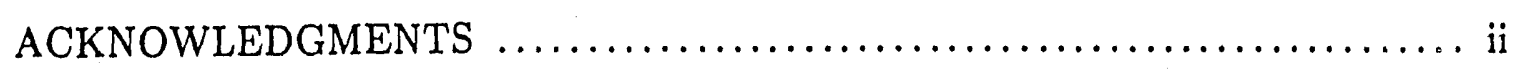

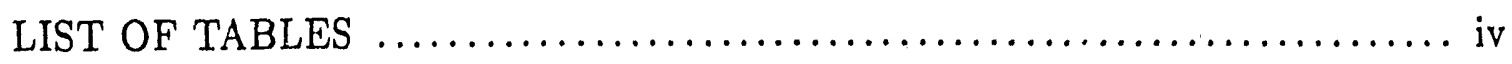

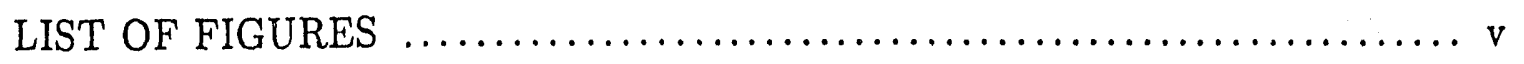

$\begin{array}{ll}\text { Chapter } & \text { Page }\end{array}$

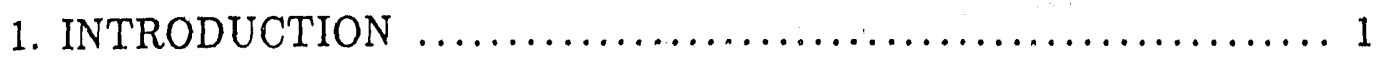

2. REVIEW OF THEORETICAL CALCULATION $\ldots \ldots \ldots \ldots \ldots \ldots 9$

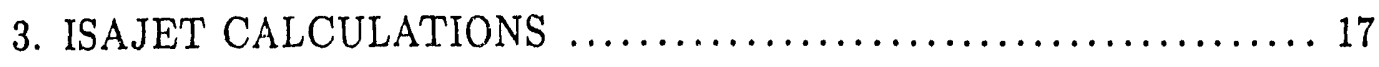

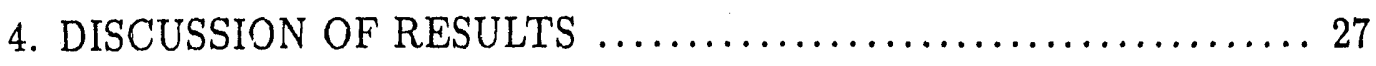

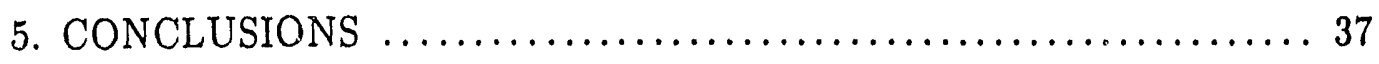

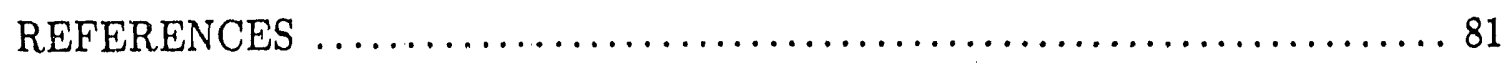




\section{LIST OF TABLES}

Table

Page

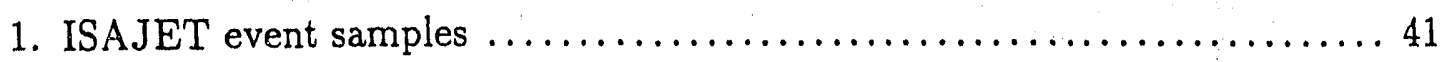

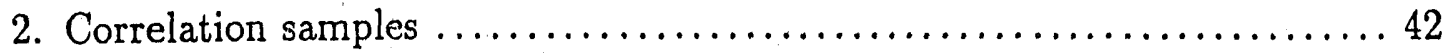




\section{LIST OF FIGURES}

Figure

Page

1. Lowest order diagrams for heavy quark production $\ldots \ldots \ldots \ldots \ldots \ldots . . \ldots 3$

2. Typical higher order diagrams involving $\ldots \ldots \ldots \ldots \ldots \ldots \ldots \ldots \ldots 44$ virtual corrections to the lowest order diagrams

3. Higher order tree level processes $\ldots \ldots \ldots \ldots \ldots \ldots \ldots \ldots \ldots \ldots \ldots \ldots . \ldots 45$ involving either gluon emission (a) or gluon splitting $(b, c, d)$

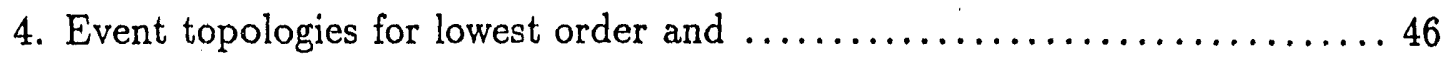
higher order processes

5. Diagrams representing gluon splitting $\ldots \ldots \ldots \ldots \ldots \ldots \ldots \ldots \ldots . \ldots 4$ events $(a 1, a 2)$ and flavor excitation $(b 1, b 2)$

6. Results of $O\left(\alpha_{s}^{3}\right)$ analytic calculations $\ldots \ldots \ldots \ldots \ldots \ldots \ldots \ldots \ldots$

7. Results from reference 28 showing the relative $\ldots \ldots \ldots \ldots \ldots \ldots \ldots \ldots 49$ contributions to the $g g \rightarrow Q X$ parton level cross-section from from lowest order $\left(f_{g g}^{(0)}\right)$ and higher order $\left(f_{g g}^{(1)}\right)$ terms (solid lines)

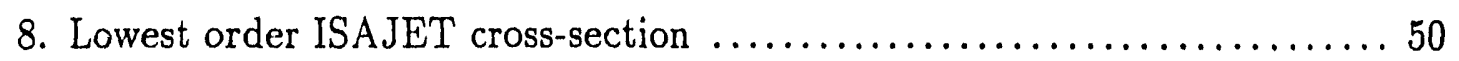
with and without gluon radiation 
9. Flavor excitation ISAJET cross-section $\ldots \ldots \ldots \ldots \ldots \ldots \ldots \ldots \ldots \ldots \ldots$ with and without gluon radiation

10. Treatment of hard scattering events 52 with a heavy flavor in the initial state

11. Differential cross-sections 53 for the processes $b q \rightarrow b X$ and $g q \rightarrow b X$ at $\sqrt{s}=630 \mathrm{GeV}$

12. Differential cross-sections 54 for the processes $b q \rightarrow b X$ and $g q \rightarrow b X$ at $\sqrt{s}=1800 \mathrm{GeV}$

13. Cross-sections from "default" ISAJET at $\sqrt{s}=630 \mathrm{GeV}$ 55

14. Cross-sections from "intermediate" ISAJET at $\sqrt{s}=630 \mathrm{GeV}$. 56

15. Cross-sections from "modified" ISAJET at $\sqrt{s}=630 \mathrm{GeV}$ 57

16. Total cross-sections for "default", "intermediate" 58 and "modified" ISAJET samples at $\sqrt{s}=630 \mathrm{GeV}$

17. K-factor at $\sqrt{s}=630 \mathrm{GeV}$ obtained from IS $\mathrm{AJET}$ samples 59

18. Cross-sections from "default" ISAJET at $\sqrt{s}=1800 \mathrm{GeV}$ 60

19. Cross-sections from "intermediate" ISAJET at $\sqrt{s}=1800 \mathrm{GeV}$ 61 
20. Cross-sections from "modified" ISAJET at $\sqrt{s}=1800 \mathrm{GeV} \ldots \ldots \ldots \ldots 62$

21. Total cross-sections for "default", "intermediate" ...............6 63 and "modified" ISAJET samples at $\sqrt{s}=1800 \mathrm{GeV}$

22. $\mathrm{K}$-factor at $\sqrt{s}=1800 \mathrm{GeV}$ obtained from ISAJET samples $\ldots \ldots \ldots \ldots 64$

23. Transverse momentum distribution from default 65 ISAJET for the $b$ quark with $\left|y_{b}\right|<1$

24. Transverse momentum distribution from default 66 ISAJET for the $b$ quark with $1<y_{b}<3$

25. Rapidity distribution from default 67 ISAJET for the $b$ quark with $p_{T, b}>10 \mathrm{GeV}$

26. Rapidity distribution from default 68 ISAJET for the $b$ quark with $p_{T, b}>20 \mathrm{GeV}$

27. Transverse momentum distribution from default ISAJET 69 for $p_{T}^{\text {pair }}$, the vector sum of the transverse momenta of the $b$ and the $\bar{b}\left(\left|y_{b}\right|<1\right)$

28. Transverse momentum distribution from default ISAJET 70 for $p_{T}^{\text {pair }}$, the vector sum of the transverse momenta of the $b$ and the $\bar{b}\left(1<y_{b}<3\right)$

29. Rapidity difference distribution of the heavy flavor pair 71 for each production mode $\left(\left|y_{b}\right|<1\right.$ and $\left.p_{T, b}>10 \mathrm{GeV}\right)$ 
30. Rapidity difference distribution of the heavy flavor pair $\ldots \ldots \ldots \ldots \ldots . \ldots 2$ for each production mode $\left(1<y_{b}<3\right.$ and $\left.p_{T, b}>10 \mathrm{GeV}\right)$

31. Rapidity difference distribution of the heavy flavor pair $\ldots \ldots \ldots \ldots \ldots 73$ for each production mode $\left(\left|y_{6}\right|<1\right.$ and $\left.p_{T, b}>20 \mathrm{GeV}\right)$

32. Rapidity difference distribution of the heavy flavor pair 74 for each production mode $\left(1<y_{b}<3\right.$ and $\left.p_{T, b}>20 \mathrm{GeV}\right)$

33. Azimuthal angle distribution of the heavy flavor pair 75 for each production mode $\left(\left|y_{b}\right|<1\right.$ and $\left.p_{T, b}>10 \mathrm{GeV}\right)$

34. Azimuthal angle distribution of the heavy flavor pair 76 for each production mode $\left(\left|y_{b}\right|<1\right.$ and $\left.p_{T, b}>20 \mathrm{GeV}\right)$

35. The strong coupling strength $\alpha_{s}$ is shown with $\ldots \ldots \ldots \ldots \ldots \ldots \ldots 77$ fixed mass and scale choice for each of the three data samples

36. The strong coupling strength $\alpha_{s}$ is shown using 78 the appropriate parameters for each of the three data samples as described in the beginning of section 3

37. EHLQ (solid line) and DFLM (dashed line) 79 gluon distribution as a function of $p_{T}$

38. EHLQ (solid line) and DFLM (dashed line) 80 bottom distribution as a function of $p_{T}$ 


\section{CHAPTER 1}

\section{INTRODUCTION}

Progress in the understanding of strong interactions has been made over the last decade at a ponderous but steady rate. Experiments have supported the predictions of perturbative quantum chromodynamics (QCD) for various processes such as Drell-Yan production of muon pairs, ${ }^{1}$ inclusive jet cross-sections, ${ }^{2}$ and direct photon events. ${ }^{3}$ Current interest in the field is centered on heavy flavor production with experiments focusing on precision measurement of $b$ quark physics processes involving $\mathrm{CP}$ violation/flavor mixing ${ }^{4}$ and also on top searches. ${ }^{5}$

The term "heavy flavor" refers to a parton with mass large compared to the QCD scale parameter $\Lambda_{Q C D} \approx 200 \mathrm{MeV}$. A heavy quark may be generated through electroweak interactions at lepton colliders or by deep inelastic scattering. At hadron colliders the production of heavy quarks takes place through QCD mechanisms. The creation of a heavy quark, such as charm, bottom, or top, through the reaction $p \bar{p} \rightarrow Q X$ is believed to occur by the interaction of the constituents ("partons") of the colliding hadrons. These constituents are the light valence quarks of the hadron and the "sea" quarks and gluons. While the electroweak interactions provide for the production of a heavy flavor at a $W Q Q^{\prime}$ or $Z Q \bar{Q}$ vertex, $Q C D$ perturbation theory only allows a heavy quark pair to be produced from a gluon vertex.

Theoretical calculations involving hadronic production of a $Q \bar{Q}$ pair assume that the cross-section can be written as a convolution of a hard scattering interaction cross-section for the constituent quarks and gluons with a product of "parton distribution functions". These functions represent the probability density of finding 
a parton of a given type at a given energy scale, $Q^{2}$, and with a certain fraction of the hadron momentum, $x$. This factorization assumption is common to many, if not all, perturbative QCD calculations of hadronic production processes.

The hard scattering cross-section is usually expressed as a perturbative expansion in $\alpha_{s}\left(Q^{2}\right)$, the scale dependent coupling strength. Each term in the series is evaluated by computing the relevant Feynman graphs which yield contributions to the given order. At the parton level, the lowest order production of a heavy quark pair in a hadronic collision is given by two processes:

$$
\begin{aligned}
& q \bar{q} \rightarrow Q \bar{Q} \\
& g g \rightarrow Q \bar{Q}
\end{aligned}
$$

Feynman diagrams representing these processes are shown in figure 1. These diagrams represent the leading order $O\left(\alpha_{s}^{2}\right)$ contributions to heavy flavor production in hadron collisions. The theory and phenomenology of such events at lowest order in QCD perturbation theory are well understood up to ambiguities involving the renormalization/factorization scale choice and the choice of parton distribution functions. ${ }^{6}$ A typical chcice of scale is set by the mass of the heavy quark, $m_{Q}$. The massive quark assures that the lowest order diagrams do not diverge and that perturbation theory is trustworthy.

The total cross-section for the above lowest order process can be studied as a function of $\sqrt{s}$ and $m_{Q}$. As the center-of-mass energy of the hadron collision increases above the scale set by the heavy quark mass, the contribution to heavy quark pair production from the gluon initiated processes increases and becomes the dominant production mode. Studies have also been made of the rapidity and transverse momentum distributions of the heavy flavor. ${ }^{7}$ Consequences of such calculations include transverse momentum distributions which peak at approximately $\left\langle p_{T, Q}\right\rangle=m_{Q}$ and fall rapidly as $p_{T, Q}>m_{Q}$.

The consideration of doubly differential cross-sections involving the heavy fla- 
vor pair leads to an investigation of momentum correlations as a probe of the QCD production mechanism. At first glance, such studies appear to be trivial in the case of lowest order production since the back-to-back nature of the final state in the parton center-of-mass frame gives simple predictions for the transverse momentum of the pair and the azimuthal angle between the two final state heavy quarks. However, the relationship of rapidities between the $Q$ and $\bar{Q}$ leads to interesting results even at the level of $O\left(\alpha_{s}^{2}\right)$ processes. Simple analytic expressions for the lowest order crosssections have been derived which are useful for studying rapidity correlations between the heavy quark and its partner. ${ }^{8}$ The interplay of the dynamics between the QCD lowest order diagrams and the parton distribution functions is reflected in positive correlation behavior between the $Q$ and $\bar{Q}$ in which the rapidity of the antiquark tends to follow that of its partner.

The subject is further enriched by the expectation that higher order production processes will contribute a non-negligible cross-section. In kinematic regimes where gluon distributions are large and gluon-gluon scattering is importani, processes where a heavy flavor pair is produced by gluon splitting in either the initial ("flavor excitation") or final state could be a significant part of the inclusive cross-section. Typical higher order processes are shown in figures 2 and 3 . These events include the virtual corrections to the Born diagrams and also incorporate the following $2 \rightarrow 3$ processes:

$$
\begin{aligned}
q \bar{q} & \rightarrow Q \bar{Q} g \\
g q & \rightarrow Q \bar{Q} q \\
g \bar{q} & \rightarrow Q \bar{Q} \bar{q} \\
g g & \rightarrow Q \bar{Q} g .
\end{aligned}
$$

Note that at this order the quark(antiquark)-gluon initial state is allowed. Such events have final state topologies which can be quite different from those predicted by lowest order processes. As figure 4 indicates, the presence of an extra jet in the final state can lead to complex events in which the $Q \bar{Q}$ pair are no longer back-to-back in the parton center-of-mass frame. These events would modify the lowest order distributions in 
rapidity and transverse momentum. In the case of correlation distributions, these changes may lead to qualitative differences in the shape of the cross-section plots.

This thesis explores those theoretical issues in heavy flavor production which are relevant to the higher order effects mentioned above. The analytic next-to-leading order $O\left(\alpha_{s}^{3}\right)$ calculation of heavy flavor production cross-sections has recently been performed by Nason, Dawson, and Ellis (NDE) and by W. Beenakker et al. ${ }^{9,10}$ This theoretical accomplishment is an excellent example of "state of the art" techniques in QCD perturbation theory being used to generwie interesting and important phenomenological results. Since the $O\left(\alpha_{s}^{3}\right)$ calculation is presented only as an inclusive distribution in the rapidity and transverse momentum of the massive quark, ${ }^{11}$ one must also turn to Monte Carlo calculations in order to study the full event structure of heavy flavor pair production. In particular, it is important to understand $Q \bar{Q}$ momentum correlations in both the Monte Carlo and in the $O\left(\alpha_{s}^{3}\right)$ calculation. The topologically nontrivial final states produced by higher order processes may serve as background to signals for other new physics. Knowledge to the relative locations in phase space of a heavy quark pair is also crucial for carrying out studies of $B$ meson mixing.

The analytic and Monte Carlo approaches employ different physical assumptions in calculating heavy flavor cross-sections. The analytic calculation includes the correct treatment of virtual processes (the Monte Carlo does not) while the Monte Carlo has provisions for multiple gluon emission-albeit approximately-which are not found in the finite order perturbative calculation. A general feature of any perturbative calculation is the treatment of singularities that occur when evaluating specific diagrams in various regions of phase space. The simplest Born diagrams have been shown in figure 1. Virtual corrections include gluon loops and vertex corrections. These processes give interference terms that contribute to the $O\left(\alpha_{s}^{3}\right)$ cross-section when they are multiplied by the lowest order amplitudes. Bremsstrahlung diagrams are those in which gluons are radiated in either the initial or final states. These diagrams are responsible for collinear divergences when the gluon and quark have 
the same direction and for infrared problems when the gluons become soft. NDE describe the various singularities which are treated in their calculation and discuss a renormalization scheme (described in detail in reference 11 ) to handle ultraviolet divergences.

Monte Carlo algorithms use various approximation techniques to avoid the singularities that plague the exact calculation. In PAPAGENO, for example, the treelevel matrix elements for $2 \rightarrow 2$ and $2 \rightarrow 3$ processes are included, and the user must provide the appropriate kinematic cut-offs when computing distributions. ${ }^{12}$ ISAJET uses a branching algorithm in order to approximate the $2 \rightarrow 3$ processes. ${ }^{13}$ Multiplying the $2 \rightarrow 2$ matrix elements by splitting functions is not a good approximation over all of phase space. ${ }^{14}$ However, the use of exact tree-level matrix elements for $2 \rightarrow 3$ processes leads to double counting problems when inserted irto a Monte Carlo with shower evolution. ${ }^{15}$ (This is why one can roughly classify a Monte Carlo code as being either a matrix element or a shower algorithm.) The shower codes incorporate some form of leading $\log$ QCD evolution of the initial and final state partons that participate in a $2 \rightarrow 2$ hard scattering. In this case $Q \bar{Q}+$ jet events, for example, would be created by gluon-gluon scattering, with subsequent evolution and eventual splitting into a $Q \bar{Q}$ pair. This is the procedure used with ISAJET in this work.

The case of bottom quark production at hadron colliders is chosen for the first attempt at studying the above topics. NDE apply their calculation to the cases of charm $\left(m_{c}=1.5 \mathrm{GeV}\right)$, bottom $\left(m_{b}=4.9 \mathrm{GeV}\right)$ and top quark $\left(m_{t}>8.9 \mathrm{GeV}\right)$ production. Since charm is the lightest quark considered, one might question the applicability of perturbative QCD in calculating production rates. Any reasonable variation of the mass will give values less than $1 \mathrm{GeV}$, at which point it is dangerous to trust perturbation theory. NDE observe that their calculated cross-sections are quite sensitive to the choice of charm quark mass and to the choice of paiton distribution functions. Other open issues are leading particle and higher twist effects on the longitudinal momentum distribution and the nuclear $A$ dependence of the crosssections. ${ }^{16}$ It appears that charm production studies will have to take into account 
several non-perturbative effects because of the relatively small mass involved. In contrast to this behavior, the QCD predictions for top quark production are expected to be quite accurate. ${ }^{9}$ The higher order terms calculated by NDE are small at CDF energies, although threshold effects appear to be non-negligible. ${ }^{17}$ In the case of bottom quark production, one has a favorable situation in which the bottom quark mass is large enough to make perturbation theory "safe" and in which data are available for comparison to the theory calculations.

The first part of 'his work consists of a review of the similarities and differences to be found when examining the two different ways of calculating heavy flavor crosssections. The parameters which enter the NDE calculation are discussed and some of the qualitative features of the analytic results are reviewed. Remarks are also made on the behavior of the analytic result in various limits and special cases. Physics issues are briefly mentioned fo: small values of the heavy quark's transverse momentum. These include threshold effects and the "semi-hard" enhancements of the cross-section due to initial state gluon emission. At large values of $p_{T, Q}$ the finite order calculation cannot account for the effects of multiple gluon radiation. NDE attempt to estimate these effects in their work and point out that the analytical calculation done to $O\left(\alpha_{s}^{3}\right)$ breaks down for $\alpha_{s} \ln \left(p_{T} / m_{Q}\right)>O(1)$. An issue to be considered is how well the ISAJET gluon radiation algorithm reproduces the large $\ln \left(p_{T} / m\right)$ corrections of the cross-section expected at large $p_{T}$. Another reason for studying the large $p_{T}$ limit involves the treatment of the so-called "flavor excitation" events. The generation of these events at high transverse momentum raises double counting issues involving heavy quark structure functions. This requires careful consideration of the two cases where the initial heavy quark is considered to be a parton generated directly from the hadron, and when the quark is generated by splitting of a partonic gluon.

After the theoretical discussion, ISAJET Monte Carlo distributions for heavy flavor ( $b$ quark) production are presented. The procedure used in this work is to split the inclusive process into event types which are kinematically and/or topologically distinct and would be recognized as such by a typical detector. In the case of heavy 
flavor production one considers lowest order $Q \bar{Q}$ production, gluon splitting events (fig. $5(\mathrm{a})$ ) in which the heavy quark pair is produced by the fragmentation of a final state gluon, and flavor excitation events (fig. $5(\mathrm{~b})$ ) in which the heavy flavor is envisioned to originate in one of the initial hadrons. Samples are generated for each process and the cross-sections are summed. Data sets were generated using three different sets of parameters, one of which reproduces the NDE conditions.

In trying to explain and interpret the results, qualitative arguments can be made concerning the effect of changes in $\alpha_{s}$ and in the structure functions. The results are sensitive to the choice of parton distributions and the expression (one or two-loop) for the strong coupling strength, $\alpha_{s}$. The NDE higher order results and the ISAJET results reproduce roughly the same shape as the lowest order calculation. The ratio of the higher order cross-section to the lowest order resuit (the " $K$ factor") ranges from 2 to 3 for the analytic calculation. Comparison to recent data ${ }^{18}$ can be made at $\sqrt{s}=$ $630 \mathrm{GeV}$ and preliminary results from the Collider Detector Facility (CDF) are also available. ${ }^{19}$ The measured cross-sections at $630 \mathrm{GeV}$ are consistent with the analytical calculation. The ISAJET cross-section is consistently larger than the analytic result at both collider energies. These results ${ }^{20}$ touch on the phenomenological issues involved when one compares theoretical cross-sections, computed either by analytic means or by Monte Carlo simulation programs, for heavy flavor production to data or when one makes predictions.

Also presented are ISAJET distributions in three correlation variables: the rapidity difference, $\Delta y_{b \bar{b}}$, the azimuthal angle, $\Delta \phi_{b \bar{b}}$, and the vector sum of the transverse momentum, $p_{T}^{\text {pair }}$. These distributions contain several features which are not found in the single particle cross-sections. Higher order contributions to the $p_{T}^{\text {pair }}$ distribution are about two orders of magnitude larger than the lowest order results at high $p_{T}^{\text {pair }}$. The $\Delta y_{b \bar{b}}$ distributions are narrower than the lowest order result by about 1 unit of rapidity and the positive correlation between the quark and the antiquark first observed in the lowest order calculation is further enhanced. The $\Delta \phi_{b \bar{b}}$ 
distributions show an increase in the cross-section near 0 degrees (by about a factor of two) when one increases $p_{T, \min }$ for the $b$ quark from 10 to $20 \mathrm{GeV}$, thus amplifying the relative contribution of the gluon splitting cross-section. One anticipates that analytic results for these distributions will verify these ISAJET observations, at least qualitatively.

The author hopes to create two impressions in the mind of the reader. First, heavy flavor production at hadron colliders is a complicated affair to understand theoretically once one goes beyond leading order in perturbation theory. No one calculational technique, i.e. exact $O\left(\alpha_{s}^{3}\right)$ perturbation theory or Monte Carlo algorithms, contains all of the possibly important physics processes, such as threshold effects or multiple gluon radiation, that are expected to occur. One should be aware of the relevant liabilities of each method when estimating rates or backgrounds. The other lesson is that correlation distributions involving both the quark and the antiquark serve to illuminate much of the higher order physics beyond relative sizes of cross-sections. 


\section{CHAPTER 2}

\section{REVIEW OF THEORETICAL CALCULATION}

The QCD-improved parton model allows hadronic cross-sections to be computed by expressing the result as a product of a hard scattering parton subprocess cross-section, $d \sigma$, computed in QCD perturbation theory up to a certain order in the strong coupling constant, $\alpha_{s}\left(\mu^{2}\right)$, and a product of parton distribution functions. These functions represent physics at momentum scales less than the factorization scale, $\mu^{2}$. Although the choice of $\mu^{2}$ is arbitrary, the final cross-section obtained from the convolution of the parton densities and $d \sigma$ is independent of $\mu$ if the calculation is carried out to all orders in perturbation theory. The evolution of the parton density, $f\left(x, \mu^{2}\right)$, and the hard scattering cross-section, $d \sigma\left(\ldots \mu^{2} \ldots\right)$, with scale change is given by renormalization group procedures. A convenient way of calculating this dependence is given by the Alterelli-Parisi formalism in which evolution is represented by gluon emission or gluon splitting. ${ }^{21}$

In keeping with the factorization procedure described above, the inclusive production cross-section of a heavy quark with rapidity $y$ and transverse momentum $p_{T}$ is given by:

$$
\frac{d^{2} \sigma(p \bar{p} \rightarrow Q X)}{d y d p_{T}^{2}}=\sum_{i, j} \int d x_{1} \int d x_{2} f_{i}^{p}\left(x_{1}, \mu^{2}\right) f_{j}^{\bar{p}}\left(x_{2}, \mu^{2}\right) \frac{d^{2} \hat{\sigma}_{i j}}{d y d p_{T}^{2}}
$$

The Nason, Dawson, and Ellis calculation of the differential cross-section $d \sigma / d p_{T} d y$ is presented in reference 11 . The total cross-section is given by integrating over $p_{T}$ and $y$. NDE present the hard scattering cross-section for inclusive production of a 
heavy quark with mass $m$ from partons $i$ and $j$ in the following form: ${ }^{9,11}$

$$
\hat{\sigma}_{i j}\left(s, m^{2}\right)=\frac{\alpha_{s}^{2}(\mu)}{m^{2}} F_{i j}\left(\rho, \frac{\mu^{2}}{m^{2}}\right)
$$

where

$$
F_{i j}\left(\rho, \frac{\mu^{2}}{m^{2}}\right)=F_{i j}^{(0)}(\rho)+4 \pi \alpha_{s}(\mu)\left[F_{i j}^{(1)}(\rho)+\bar{F}_{i j}^{(1)}(\rho) \ln \left(\frac{\mu^{2}}{m^{2}}\right)\right]+O\left(\alpha_{s}^{2}\right)
$$

and $\rho=4 m^{2} / s$. The $F_{i j}^{(0)}$ term is the lowest order contribution to $F_{i j}$ from $g g$ and $q \bar{q}$ initial states $\left(F_{g q}^{(0)}=0\right)$; this part is dependent on the renormalization scale, $\mu$, only in the evaluation of the coupling constant $\alpha_{s}$. The $F_{i j}^{(1)}$ term represents the actual calculation of higher order diagrams, while the $\bar{F}_{i j}^{(1)}$ term is calculated from the lowest order $F^{(0)}$ contributions using Altarelli-Parisi splitting functions.

The mass of the heavy flavor occurs in both the matrix elements and the phase space factor in the exact calculation. Note that the hard scattering cross-section varies as $1 / m^{2}$ (the $F_{i j}$ 's are dimensionless); the mass dependence of the parton luminosity makes the drop-off in cross-section even steeper. For these Born processes, the mass sets a lower cutoff for the momentum exchange in the propagator term. Perturbation theory should be valid for $m_{Q}>\Lambda_{Q C D}$.

The expression for $\alpha_{s}$ depends on the number of flavors treated as light (massless) and on the masses of the heavy flavors, which are thresholds in the $Q^{2}$ evolution. The flavor thresholds in $\alpha_{s}$ and in the parton distributions are described by NDE. The two-loop expression ${ }^{22}$ for $\alpha$, that is used in the NDE calculation is

$$
\alpha_{s}\left(Q^{2}\right)=\frac{12 \pi}{\left(33-2 N_{e f f}\right) \ln \left(\frac{Q^{2}}{\Lambda_{e f f}^{2}}\right)}\left[1-6 \frac{153-1^{\prime} J N_{e f f}}{\left(33-2 N_{e f f}\right)^{2}} \frac{\ln \ln \left(\frac{Q^{2}}{\Lambda_{e f f}^{2}}\right)}{\ln \left(\frac{Q^{2}}{\Lambda_{e f f}^{2}}\right)}\right],
$$

where $N_{t f f}$ is the number of flavors such that $m_{q}^{2}<Q^{2}$, and $\alpha_{s}$ is continuous. (Here, $\Lambda_{4}=260 \mathrm{MeV}$.) 
The confidence in the validity of any perturbative QCD calculation for the purpose of phenomenological predictions or comparison to data depends on an understanding of the theoretical uncertainties that arise. One issue to be consiciered is the choice of scale, $Q^{2}$. When the total bottom quark cross-sections for both the lowest order and next-to-leading order results are plotted as a function of scale, ${ }^{9}$ one finds that the next-to-leading order result becomes unstable and increases as $Q$ goes to 0 for $Q<5 \mathrm{GeV}$. This value happens to be a natural lower bound on the scale set by the heavy quark mass. One also observes less scale dependence as $Q$ increases; this indicates that the choice of a $p_{T, Q}$ dependent function for the choice of scale in the calculation of differential distributions is a reasonable one. For the differential cross-section NDE choose $Q^{2}=p_{T}^{2}+m_{Q}^{2}$, and multiply $Q$ by factors ranging from 0.5 to 2 in order to test the sensitivity of their results to scale choice.

The choice of parton densities must also be specified. NDE use two-loop structure functions in their calculation to maintain consistency with the $O\left(\alpha_{s}^{3}\right)$ computation of the hard scattering cross-section. The differences between the EHLQ1 (one-loop) ${ }^{23}$ and the DFLM (two-loop) ${ }^{24}$ distributions that are relevant to $b$ prociuction will be studied when interpreting the results. Attention is focused on the gluon and bottom quark distributions in the hadron. It should be noted that for very small values of Bjorken $x$ the calculation of the parton densities becomes unreliable. Levin, Ryskin, and Shabelsky ${ }^{25}$ have calculated the effect of saturation on the charm and bottom cross-sections. Rescattering is important only for very small values of transverse momentum- $p_{T, b}<7 \mathrm{GeV}$ at the Tevatron-and leads to a decrease of the cross-section in these kinematic regions.

Recent work by J. C. Collins and R. K. Ellis ${ }^{26}$ investigates the effect of initial state gluon radiation in the "semi-hard" region where $\Lambda_{\overline{M S}}<Q<<\sqrt{s}$. The total bottom quark cross-sections are found to be larger than even the higher order results. The differential distributions are not yet available, but one might conservatively estimate that the resummation enhancements would be important for transverse momenta within an order of magnitude of the heavy quark mass. This region of phase 
space allows for small Bjorken $x$ of the initial state partons. These small $x$ effects are not present in either the leading logarithm Monte Carlo algorithms or the analytic calculation although Collins and Ellis claim their forrualism can be adapted to higher order calculations.

Higher order contributions to the total cross-section are found to be important in two regions of parton center-of-mass energy: near threshold and very far above threshold (fig. 6(a)). The threshold effects are ascribed to initial state gluon radiation and to Coulomb enhancement from virtual gluon exchange between the heavy quarks. There is a $\left(\frac{1}{v}\right)$ divergence where $v$ is the velocity of the $Q \bar{Q}$ pair in the parton center-of-mass. For $O\left(\alpha_{s}^{2}\right)$ processes, as $\hat{s} \rightarrow 4 m_{Q}^{2}$, the Born cross-section vanishes. However, the $g g \rightarrow Q \bar{Q} g$ cross-section has a finite contribution at $\hat{s}=4 m_{Q}^{2}$ due to the Coulomb singularity coming from virtual gluon exchange between the massive quarks (fig. 2(b)). The Monte Carlo study presented in this work does not explore these threshold issues; the flavor excitation and gluon splitting samples were produced with a $p_{T, b}$ cut just above $2 m_{b}$.

The enhancement at large $\hat{s}$ seen in figure $6(a)$ is created by higher order processes having $\hat{t}$-channel exchange of a spin 1 gluon. Lowest order diagrams only have spin $1 / 2$ exchange of the heavy flavor in addition to the annihilation diagrams. This increase in the cross-section carries over to the differential distributions as well. Upon computing transverse momentum and rapidity distributions, NDE find differential cross-sections uniformly larger than the lowest order result. Only the normalization, not the shape, of the heavy flavor differential cross-section appears to change (fig. $6(\mathrm{~b}))$.

Several features of the higher order calculation are not reproduced by current Monte Carlo codes. One has already seen this for the case of threshold enhancements of the cross-section. Another facet of the calculation which is difficult to include in a Monte Carlo program is the interference between various diagrams-an $O\left(g^{2}\right) g g \rightarrow$ $Q \bar{Q}$ process multiplied by an $O\left(g^{4}\right)$ higher order diagram with virtual corrections, for 
example. An example of the physics consequences of ignoring such interference terms is given by the calculation of charge asymmetry. ${ }^{27} \mathrm{NDE}$ point out that $d \sigma_{b} / d y_{b}$ is not equal to $d \sigma_{5} / d y_{\bar{b}}$ for their analytic $O\left(\alpha_{s}^{3}\right)$ result. ${ }^{11}$ To this order in perturbation theory interference diagrams which include a heavy quark loop lead to a small charge asymmetry in the rapidity distributions. Such effects would not be seen in present-day Monte Carlo calculations.

Attempts have been made to understand how well the Monte Carlo scheme reproduces the exact calculation. Meng et al. have derived approximate analytic expressions for the differential cross-sections from initial state gluon bremsstrahlung (ISGB), final state gluon splitting (FSGS), and flavor excitation. ${ }^{28}$ The ISGB contributions appear to describe the threshold effects of the analytic calculation, and the sum of the three processes at large $\eta=\frac{\hat{s}-4 m_{b}^{2}}{4 m_{b}^{2}}$ also appears to reproduce the NDE results. However, there is an intermediate region, $0.2<\eta<200$, where the sum overestimates the complete calculation (fig. 7). This region is where most current Monte Carlo calculations operate. For example, the distributions presented in this work have $p_{T, b}$ between 12 and $80 \mathrm{GeV}$ which yield $\eta$ values between .5 and 60 if one takes $\hat{s}$ approximately equal to $p_{T, b}^{2}$.

Gluon radiation at large $p_{T, Q}$ may also affect cross-sections and correlation distributions. NDE give a prominent warning that for $p_{T} \gg m_{Q}$, logarithmic terms of the form $\ln \left(p_{T}^{2} / m^{2}\right)$ lead to theoretical uncertainty. In this limit, the heavy flavor is effectively massless leading to collinear divergences. These effects are estimated in the limit where $p_{T}$ is fixed ard $m_{Q} \rightarrow 0$ using Altarelli-Parisi splitting functions. To obtain the complete leading order effect one must sum all $\alpha_{s}^{2}\left(\alpha_{s} \ln \left(p_{T} / m\right)\right)^{n}$ and $\alpha_{s}^{3}\left(\alpha_{s} \ln \left(p_{T} / m\right)\right)^{n}$ terms. NDE studied the behavior of these logarithmic terms in the massless limit and discussed some of the resummation problems that arise. ${ }^{11}$ Such issues lead to an investigation of the role of gluon radiation algorithms in the calculation of heavy flavor cross-sections. The high $p_{T}$ limit in ISAJET is marked by increasing gluon radiation which also scales as $\ln p_{T}$. At high $p_{T}$ the QCD evolution 
actually may account for some of the physics due to the large logarithms that cause the breakdown of the analytical calculation.

It would be a cirallenge to investigate how well the QCD branching algorithms reproduce these higher order terms in the limit of large $p_{T} / m_{Q}$. The lowest order $2 \rightarrow 2$ samples generate $b \bar{b}$ pairs using the cross-sections given by the simple Feynman diagrams presented in figure 1 . The momentum distribution of a $b$ quark in the final state is modified by the gluon radiation algorithm in ISAJET. This result is plotted in figure 8. Similar effects are observed for the flavor excifation sample as shown in figure 9. The net effect is to slightly decrease the cross-section at large values of transverse momentum and to increase the cross-section for low values. This effect appears to grow as the transverse momentum of the heavy flavor increases. Cudell et al. have compared cross-sections resulting from different showering mechanisms in various Monte Carlos. ${ }^{29}$ At $p_{T, b}=1000 \mathrm{GeV}$ there can be an order of magnitude difference in cross-section for different Monte Carlo calculations.

The treatment of flavor excitation events in the Monte Carlo differs from their interpretation in the analytic calculation. Flavor excitation diagrams are not included in the NDE calculation. They are considered to be higher order processes in which the $b$ quark is formed from the splitting of an initial state gluon. A qualitative argument which justifies this omission is given in reference 30 . The major point is that for small values of the $\hat{t}$-channel gluon momentum, $q^{2}<m_{Q}^{2}$, the flavor excitation contribution is accurately represented by the initial state gluon splitting diagrams. The $1 / q^{4}$ divergence exhibited in the $2 \rightarrow 2$ diagram is not present in the sum of the higher order diagrams. The NDE approximation should therefore be valid for calculations of the total cross-section in which the low $q^{2}$ region contributes to the bulk of the cross-section. However, for differential distributions at high $p_{T, Q}$, such an approximation may not hold, since $q^{2} \gg m_{Q}^{2}$. Monte Carlo calculations of heavy flavor cross-sections do include events where the incoming parton is heavy.

Concern arises over possible double counting in the treatment of flavor cxcitation; this raises theoretical issues related to heavy quark distribution functions. 
One can envision the $b$ quark that participates in the hard scatter originating directly from the hadron (fig. 5(bl)), or created by gluon splitting in the initial state (fig. 5(b2)). The NDE calculation includes only the latter possibility, while the Monte Carlo flavor excitation samples are made using the former; this suggests that the results should be added. The possibility of double ccunting arises, however, when the cross-sections are added to form the total inclusive distribution. Those $2 \rightarrow 3$ events where an initial-state gluon splits to a collinear $b$ quark look like Monte Carlo flavor excitation events. To correct for double counting, one must subtract the contribution of those events in which the $b$ quari: is produced collinearly. The procedure is represented schematically in figure $10(\mathrm{~b})$. The subtraction term portrayed by the "cut" diagram is meant to remove possible double counting. (This is the approach advocated in referen.ce 31 for treating processes like $t \bar{b} \rightarrow$ Higgs. $^{31,32}$ ) Since ISAJET generates events which are represented by the initial bottom quark diagrim, the "error" is estimated by calculating the initial gluon splitting term minus the collinear term. Similarly, the NDE approximation can be checked by calculating the difference in cross-sections represented by the first and the third diagrams in figure 10(b).

As a check of NDE's claim that flavor excitation events can be treated as higher order processes, one can compare cross-sections computed from simple flavor excitation diagrams with a "subtraction" term. The results at UA1 and CDF energies are shown in figures 11 and 12. EHLQ1 distributions are used and the scale choice is $Q^{2}=p_{T, b}^{2}+m_{b}^{2}$. These plots compare the cross-sections computed from the processes $b q \rightarrow b X(b q$ scattering) and $g q \rightarrow b X$. The latter calculation essentially consists of a $g \rightarrow b \bar{b}$ splitting function multiclied by a $b q$ scattering cross-section. These processes represent the first and third diagrams in figure $(10 \mathrm{~b})$ with the initial state spectator gluon replaced by a quark line The two cross-sections diverge slightly at $p_{T, b}=$ $80 \mathrm{GeV}$ with the bottom quark parton distribution functirss leading to the larger value. This effect would tend to become more important at larger values of $\sqrt{s}$ and $p_{T, b}$. In the regions plotted, it appears that the NDE approach is valid. The inclusive cross-section can be accurately computed using perturbative QCD in which the initial 
partons are light.

The goal of this section has been to examine possible sources of discrepancy between the analytic and Monte Carlo calculations of bottom quark production crosssections. The arguments presented here indicate, but do not conclusively prove, that for the samples used in this work only the neglect of the interference terms in the Monte Carlo calculation can lead to a major difference between the two approaches. Effects due to threshold behavior, high $p_{T, b}$ gluon radiation, and heavy quark structure functions appear to be numerically small for the ISAJET samples generated here. However, one must mention the possible importance of the "semi-hard" initial state gluon emission calculations. This physical effect is not incorporated in either the analytic calculations or in the Monte Carlo. 


\section{CHAPTER 3}

\section{ISAJET RESULTS}

The purpose of this section is to compare ISAJET results with the analytical distributions for heavy flavor production presented by Nason, Dawson, and Ellis (NDE). ${ }^{11}$ Specifically, bottom quark production is studied at $\sqrt{s}=630 \mathrm{GeV}$ and 1800 $\mathrm{GeV}$ in proton-antiproton collisions. Results are presented in terms of the integral transverse momentum distribution $\sigma_{T}\left(p_{T, b}>p_{T, \min }\right)$, which is computed by integrating the analytical result for $d \sigma / d y d p_{T}$ over the appropriate rapidity range, and over the transverse momentum of the $b$ quark from $p_{T, \min }$ to infinity. Distributions of $\sigma_{T}\left(p_{T, b}>p_{T, \min }\right)$ are presented using three different parameter sets. The parameters varied are the mass of the bottom quark $m_{b}$, and the functional expressions for the strong coupling constant $\alpha_{s}$, the hard scattering scale $Q^{2}$, and the parton distributions $f\left(x, Q^{2}\right)$. In order to compare ISAJET cross-sections with the NDE calculation next-to-leading order DFLM structure functions are used in conjunction with the two-loop expression for $\alpha_{s}$ in one of the parameter sets. This set is referred to as "modified" ISAJET. To compare ISAJET cross-sections with data at $630 \mathrm{GeV}$ and with previous calculations the EHLQ1 structure functions are used together with a simple lowest order expression for $\alpha_{s}$. This data set is called "default" ISAJET. Finally, in order to studys the effect of changing the strong coupling constant and the structure functions a second EHLQ1 data set is generated using a one-loop $\alpha_{s}$ (in $\overline{M S}$ scheme) which is continuous across quark mass thresholds as in the DFLM sample. The $b$ mass and the scale choice are also taken to agree with the DFLM set. This set is called "intermediate" ISAJET in the figures and discussion.

Once the total cross-sections have been computed, one can begin to make a 
more detailed study of event topology. In particular, at the parton level, distributions in $p_{T}^{\text {pair }}, \Delta \phi_{Q Q}$, and $\Delta y_{Q Q}$ can be generated. These distributions provide quantitative information on the dynamics of higher order effects in heavy flavor production, particularly when one studies the effects of transverse momentum and rapidity selections for the heavy flavor. The default ISAJET sample is used for this study.

The defauit values of the ISAJET Monte Carlo are:

$$
\begin{gathered}
\text { EHLQ1 structure functions }\left(\Lambda_{Q C D}=200 \mathrm{MeV}\right), \\
m_{b}=4.9 \mathrm{GeV}, \\
Q^{2}=\max \left(4 m_{b}^{2}, 2 \hat{s} \hat{t} \hat{u} /\left(\hat{s}^{2}+\hat{t}^{2}+\hat{u}^{2}\right)\right) \\
\alpha_{s}\left(Q^{\prime}\right)=\frac{12 \pi}{\left(33-2 N_{e f f}\right) \ln \frac{Q^{2}}{\Lambda_{Q C D}^{2}}} \\
N_{c f f}=4+\frac{Q^{2}}{Q^{2}+m_{b}^{2}}+\frac{Q^{2}}{Q^{2}+m_{t}^{2}}
\end{gathered}
$$

The top quark mass in ISAJET is taken to be $40 \mathrm{GeV}$. The expression for $\alpha$, depends on the number of flavors treated as light (massless) and on the masses of the heavy flavors, which are thresholds in the $Q^{2}$ evolution. The choices of $Q^{2}$ and of the functional form for $N_{\text {eff }}$ are historical artifacts in the default version of ISAJET. Different choices would be made if the program were being developed today.

Samples were also generated using an expression for $\alpha_{\text {, which is continuous }}$ across mass thresholds and also uses the same mass and scale as the DFLM sample. For this "intermediate" set the values are:

$$
\begin{aligned}
& \text { EHLQ1 structure functions }\left(\Lambda_{Q C D}=200 \mathrm{MeV}\right), \\
& \qquad \begin{array}{l}
m_{b}=4.75 \mathrm{GeV} \\
Q^{2}=m_{b}^{2}+p_{T, b}^{2}
\end{array}
\end{aligned}
$$




$$
\alpha_{s}\left(Q^{2}\right)=\frac{12 \pi}{\left(33-2 N_{e f f}\right) \ln \frac{Q^{2}}{\Lambda_{n}^{2}}} .
$$

in which $N_{e f f}$ is simply the number of flavors such that $m_{q}^{2} \leq Q^{2}$, and $\Lambda_{\text {eff }}$ is recomputed to maintain continuity of $\alpha$ across flavor thresholds. Thus,

$$
\alpha_{s}\left(Q^{2}\right)=\left\{\begin{array}{rr}
12 \pi / 25 \ln \left(\frac{Q^{2}}{\Lambda_{t}^{2}}\right), \quad Q^{2}<m_{b}^{2} \\
12 \pi / 23 \ln \left(\frac{Q^{2}}{\Lambda_{s}^{2}}\right), \quad m_{b}^{2} \leq Q^{2}<m_{t}^{2}
\end{array},\right.
$$

where $\Lambda_{5}=\Lambda_{4}\left(\Lambda_{4} / m_{b}\right)^{2 / 23}$.

DFLM structure functions are also used for the comparison to the analytic NDE results. The parameters for the "modified" ISAJET sample were:

$$
\begin{gathered}
\text { DFLM structure functions }\left(\Lambda_{Q C D}=260 \mathrm{MeV}\right), \\
\qquad \begin{array}{r}
m_{b}=4.75 \mathrm{GeV} \\
Q^{2}=m_{b}^{2}+p_{T, b}^{2}
\end{array} \\
\alpha_{s}\left(Q^{2}\right)=\frac{12 \pi}{\left(33-2 N_{e f f}\right) \ln \left(\frac{Q^{2}}{\Lambda_{e f f}^{2}}\right)}\left[1-6 \frac{153-19 N_{e f f}}{\left(33-2 N_{e f f}\right)^{2}} \frac{\ln \ln \left(\frac{Q^{2}}{\Lambda_{e f l}^{2}}\right)}{\ln \left(\frac{Q^{2}}{\Lambda_{e f f}^{2}}\right)}\right]
\end{gathered}
$$

where, again, $N_{\text {eff }}$ is the number of flavors such that $m_{q}^{2}<Q^{2}$, and $\alpha_{s}$ is continuous. (Here, $\Lambda_{4}=260 \mathrm{MeV}$.) The momentum scale, $Q^{2}$, was always computed from values $\left(p_{T}, \hat{s}, \hat{t}, \hat{u}\right)$ taken from the $2 \rightarrow 2$ hard scattering, before any QCD evolution occurs. It is important to keep this in mind for the gluon splitting samples, because the transverse momentum of the $b$ quark was not used to evaluate $Q^{2}$ here.

The choice of momentum scale for $\alpha_{s}$ and the parton distributions essentially relies on $p_{T, Q}$ and $m_{Q}$, the two natural scales available. In this work ISAJET samples 
are generated for two choices of scale: the NDE scale, $Q^{2}=p_{T}^{2}+m_{Q}^{2}$, and the default ISAJET scale, which is given by $Q^{2}=\max \left(4 m_{Q}^{2}, 2 \hat{s} \hat{t} \hat{u} /\left(\hat{s}^{2}+\hat{t}^{2}+\hat{u}^{2}\right)\right)$. As $p_{T}$ grows, both scales are asymptotically proportional to $p_{T}^{2}$ (with the constant of proportionality equal to $4 / 3$ in the tefault ISAJET case).

Samples of events were generated for the following three processes:

$$
\begin{array}{cccl}
q+\bar{q}(g+g) & \rightarrow & b \bar{b} & \text { (lowest order QCD) } \\
(g, u, d, s)+(g, u, d, s) & \rightarrow & g+X & \\
& & \llcorner b \bar{b} & \text { (gluon splitting) } \\
b(\bar{b})+(g, u, d, s) & \rightarrow & b(\bar{b})+X & \text { (flavor excitation) }
\end{array}
$$

To compare with the analytical inclusive cross-section one simply adds the three different cross-sections- $b \bar{b}$ lowest order, gluon splitting, and flavor excitation-bin by bin. As mentioned in the previous section, this approximation scheme does not include interference effects from loop diagrams. One justification for making this approximation is that at high $p_{T}$ each of the three above processes is kinematically distinct, and therefore interference terms between any two processes become small. Lowest order events produce the pair back-to-back in the parton center-of-mass frame, while gluon splitting leads to both heavy quarks being close together in phase space. Flavor excitation processes are characterized by one quark being scattered at large transverse momentum with the other having small transverse momentum and often going down the beam pipe. In any case, the results at low $p_{T}$ depend on the (unphysical) cut-offs imposed to avoid singularities; most attention is devoted to results at higher $p_{T}$.

Some of the physics approximations that are assumed in calculating gluon splitting or flavor excitation cross-sections were explained in the previous chapter, but the discussion there does not describe why such approximations need to be made when running ISAJET. Gluon radiation from the lowest order diagrams would seem to provide contributions from what could be considered flavor excitation and gluon splitting processes, depending on which virtual parton is responsible for the exchange 
of the hard scattering transverse momentum. Unfortunately, ISAJET cannot compute the full contribution from the diagram in figure $3(b)$ by treating it as a lowest order $g g \rightarrow Q \bar{Q}$ process with a gluon radiated from the initial state. This is because the Sjöstrand algorithm ${ }^{33}$ always orders the momentum transfers in the chain of initial state radiation in decreasing sequence from the scale of the $2 \rightarrow 2$ hard scattering. Therefore the flavor excitation sample is needed to cover all of phase space. The lowest order diagrams allow for the heavy quark to have transverse momentum larger than the gluon off which the heavy quark scatters, while in the flavor excitation events one has the hard momentum transfer carried by the recoil gluon. A similar argument can be made to explain why the gluon splitting events cannot be treated by $Q \bar{Q}$ lowest order events together with shower algorithms.

Typical diagrams that occur in the higher order NDE calculation are shown in figures 2 and 3 . Figure $3(\mathrm{a})$ can be viewed as gluon radiation after production of a $b \bar{b}$ pair; for soft or nearly collinear gluons, such events will be generated in the lowest order sample. The diagram in figure 5(b1) represents ISAJET flavor excitation. Those processes which generate the heavy flavor pair from final state gluon splitting, as shown in figures $3(\mathrm{c})$ and $3(\mathrm{~d})$, are generated in the ISAJET $g, u, d, s$ samples. The cross-sections are computed by convoluting gluon splitting with the $2 \rightarrow 2$ scattering of light partons (fig. 5(a2)). It is possible for the $b$ quark to be produced after a cascade of gluon radiation. In this case, the transverse momentum of the $b$ quark can be significantly less than that of the hard scattering.

The $b \bar{b}$ and flavor excitation samples are created using the standard TWOJET subroutine in ISAJET, with final state partons specified to be $b$ quarks. For lowest order QCD pair production the final state is a $b \bar{b}$ pair.

The flavor excitation samples require only that one of the outgoing partons from the hard scatter be a bottom quark. Flavor excitation cross-sections as produced by ISAJET must be doubled to account for either final state parton being a $b$ quark. Here the $b$ quark distribution is inherent in the heavy flavor component of 
the structure functions. This distribution is used to assign the weight to the event before any initial state evolution is done. The subsequent backward radiation which is done modifies only the topology of the event structure-not the cross-section. The details of both initial and final state QCD evolution are described in the ISAJET documentation ${ }^{13}$ and will not be discussed here.

The gluon splitting events are produced by ISALEP, a modified form of ISAJET in which two light partons scatter before QCD evolution is allowed to generate a pair. In the subroutine that does the QCD evolution of the final state, events are counted in which a gluon converts into a $b \bar{b}$ pair. No conditions are placed on the rapidity or transverse momentum of the $b$ quark, or of the lepton from the resulting weak decay, although provisions are made in the modified code for such selections. If the specified heavy flavor pair is not produced after ten fragmentations, the event is discarded. It should be noted that $Q C D$ evolution can produce a $Q \bar{Q}$ pair in either the final or initial state radiation, for a $2 \rightarrow 2$ hard scatter of light partons. The $b \vec{b}$ pairs made in the initial state evolution typically have low transverse momentum and play a negligible role in the results presented.

The DFLM flavor excitation samples require changes and insertions in the body of the code in order to call the new set of parton distributions. $\Lambda_{Q C D}$ must be set in the input parameter file, not in the common block used within the program. The mass of the $b$ quark is changed in the appropriate DATA statement inside ISAJET. The scale and expression for $\alpha_{s}$ are changed within subroutine TWOKIN and the DFLM distributions themselves are inserted in subroutine STRUC. For the "intermediate" sample one need only change the mass, scale, and expression for $\alpha_{s}$ in the ISAJET code.

After event samples are generated over several ranges of transverse momentum, the analysis of each sample is done using a version of ISAPLT. The analysis code must be modified to create the desired histograms for each momentum range. Results can be concatenated using the HBOOK inspired HIST program. It is important to add the overflow value to the integrated cross-section when running HIST to assure that 
the high $p_{T}$ results are not systematically small. All fits to histograms were made using the TOPDRAWER graphics routine.

In the analysis we only count $b$ 's (not $\bar{b} \bar{b}$ 's) that occur as "stable" particles after applying the ISAJET gluon branching algorithm. The QCD branching algorithms may produce more than one pair of heavy flavors. To check the importance of this effect, one plots total number of $b$ 's per event. Those events with two or more pairs never occurred more frequently than about 1 in 200 times before any rapidity cuts were applied. For a test sample, $d \sigma / d p_{T, b}$ was computed for all $b$ 's, and this distribution was compared to events with only 1 bottom quark and with the distribution of the highest $p_{T} b$ quark seen per event. No significant differences could be observed within statistical errors in either the shape of the distributions or in the normalized values of the differential cross-sections. The analysis therefore counts all the $b$ quarks generated in the event which satisfy the rapidity cut. All of the following distributions were made using such an inclusive procedure.

Each sample (except as noted) has 1000 events per $2 \mathrm{GeV}$ bin in transverse momenturn of the hard scattering, $p_{T}^{\text {part }}$. This variable is the bottom quark transverse momentum generated in the lowest order and flavor excitation samples before any gluon radiation occurs. In the gluon splitting samples, $p_{T}^{\text {part }}$ is the transverse momentum scale of the $2 \rightarrow 2$ hard scattering of the light initial state $g, u, d, s$ partons. Table 1 contains the range of $p_{T}^{\text {part }}$ for each sample, and the total cross-section for the samples and their sums. One imposes $\left|y_{b}\right|<1.5$ for the $630 \mathrm{GeV}$ sets and $\left|y_{b}\right|<1$ for the $1800 \mathrm{GeV}$ samples in order to compare with the NDE results. The efficiency of the rapidity cuts is also given.

The gluon splitting data sets are binned according to the $p_{T}$ of the hard scattering parton $g, u, d, s$ before branching occurs to the $b \bar{b}$ pair. The upper limits are chosen high enough to get high-statistics integrated $\sigma_{t o t}\left(p_{T, b}>p_{T, \min }\right)$ data out to $p_{T, b}=80 \mathrm{GeV}$ for collider energies of $\sqrt{s}=630 \mathrm{GeV}$ and $\sqrt{s}=1800 \mathrm{GeV}$. This required that $p_{T}^{\text {part }}$ be taken out to about twice the $b$ quark transverse momentum. 
For $b \bar{b}$ production a non-zero value of $p_{T, \min }$ must be set to avoid numerical errors in ISAJET. For the other two production modes, the limit is set by kinematics. The $b \bar{b}$ pair produced by gluon splitting in either the initial or final state demands that $p_{T}>2 m_{b}$.

Figures 13 through 15 show the integrated cross-section $\sigma_{t o t}\left(p_{T, b}>p_{T, \min }\right)$ as a function of $p_{T, \min }$ for $\sqrt{s}=630 \mathrm{GeV}$ with each figure representing a different parameter set. Results are presented for the three individual processes of lowest order $b \bar{b}$ production, flavor excitation, and gluon splitting, respectively. The solid line in each plot is the sum of the cross-sections obtained from the three production modes, which is taken to be the total inclusive distribution produced by ISAJET for a given set of masses, scales, and structure functions. Figure 16 superimposes these ISAJET total cross-sections on the analytical result of NDE. The measured values published by UA1 are also displayed. ${ }^{18}$

UA1 base their bottom quark cross-section measurement on four sets of muon samples. The integral cross-section $\sigma\left(p_{T, b}>p_{T, m i n}\right)$ is obtained for each sample and the total cross-section is obtained by extrapolating to zero transverse momentum. The $J / \Psi$ sample studies those high $p_{T} J / \Psi$ 's which are embedded in jets. These are assumed to come from $b$ decay. There is also a high-mass dimuon sample with $p_{T}(\mu \mu)$ larger than $3 \mathrm{GeV}$ and $M(\mu \mu)>6 \mathrm{GeV}$. Most of these events are created with the $b$ quarks widely separated; higher order events are suppressed in this sample. The low mass dimuon sample has $2<M(\mu \mu)<6 \mathrm{GeV}$. Finally, the inclusive muon sample $\left(p_{T, b}>6\right)$ is measured in six momentum ranges of $5 \mathrm{GeV}$ bins from 10 to $40 \mathrm{GeV}$ for the transverse momentum of the muon. The hard bottom quark fragmentation functions make any $p_{T}$ cut on the transverse momentum of the lepton biased in favor of the bottom events over charm. The rapidity cut for all samples is $\left|y_{\mu}\right|<1.5$.

Figures 18 through 21 present similar plots at the Tevatron energy of $\sqrt{s}=$ $1800 \mathrm{GeV}$. Preliminary results from CDF are also shown. ${ }^{19}$

Figures 17 and 22 are the $K$ factor results from our samples at 630 and 1800 
$\mathrm{GeV}$, respectively. This is the ratio of the total inclusive result over the lowest order $2 \rightarrow 2$ cross section as a function of $p_{T, \min }$. For the ISAJET plots the total cross-section is divided by a " $2 \rightarrow 2$ lowest order" cross-section for $b \bar{b}$ production which is not just the tree-level result, but includes initial-state gluon radiation. These plots also have the ratio obtained from the NDE results. The analytical expression is simply defined as:

$$
K=\frac{O\left(\alpha_{s}^{2}\right)+O\left(\alpha_{s}^{3}\right)}{O\left(\alpha_{s}^{2}\right)}
$$

where $O\left(\alpha_{s}^{2}\right)$ represents the Born cross-section and the $O\left(\alpha_{s}^{3}\right)$ termi comes from the higher order calculation. To obtain these curves the NDE total cross-sections at the appropriate energy are divided by the lowest order DFLM cross-section. An accurate reproduction of the analytical result for the $K$ factor demands that one use the $2 \rightarrow 2$ hard scattering cross-sections before the QCD evolution of the final state occurs. As figure 8 indicates, at high $p_{T}$ the value of the Born cross-section may differ by as much as 30 percent when compared to the lowest order cross-sections given by ISAJET after gluon radiation.

Figures 23 and 24 show the transverse momentum distribution of the $b$ quark from the default ISAJET sample for two different rapidity regions: $\left|y_{b}\right|<1$ and $1<y_{b}<3$. Figures 25 and 26 show the rapidity distribution of the $b$ quark with transverse momentum cuts of 10 and $20 \mathrm{GeV}$ respectively. (No conditions are placed on the $\bar{b}$ quark.) These distributions will help in interpreting the correlation plots which follow. As table 2 shows, the cut at $20 \mathrm{GeV}$ serves to increase the relative size of the gluon splitting and flavor excitation cross-sections so that they are slightly larger than the lowest order contribution. It is interesting to observe that the relative sizes of the total cross-sections are relatively insensitive to the different rapidity selections on the bottom quark.

Three variables are chosen to study the correlation behavior of heavy flavor pair production: the vector sum of the transverse momentum, the rapidity difference, and the azimuthal angle in the transverse plane between the $b$ and its antiquark. 
The parton level plots show the distributions for each production mode and the total inclusive distribution that results when the lowest order, flavor excitation, and gluon splitting samples are added bin by bin as in the integrated cross-section plots. Figures 27 and 28 are the $p_{T}^{\text {pair }}$ distributions for $p_{T, b}>10 \mathrm{GeV}$ and with the two different selections on rapidity. The next four figures show the rapidity difference distributions for all combinations of rapidity and transverse momentum selections. Figures 33 and 34 are the azimuthal angle distributions for $\left|y_{b}\right|<1$ and with the two different selections on transverse momentum. Sample $\varepsilon^{+}$ntistical error bars as calculated by ISAJET are included in these figures.

The only true subtlety to be aware of when binning the correlation histograms occurs when one plots the $\Delta y$ distributions for the flavor excitation cross-sections. Since the samples were generated with the $b$ quark coming only from the proton, one had to double the cross-section obtained from the Monte Carlo. To treat the rapidity difference distributions correctly one must instead add the "mirror" events that represent the $b$ quarks that would come from the antiproton. To do this one plots $y_{5}-y_{b}$ for $b$ quarks having rapidity $-1>y_{b}>-3$. 


\section{CHAPTER 4}

\section{DISCUSSION OF RESULTS}

Here is discussed the comparison of the three ISAJET samples with each other, and with the analytic results of NDE; the phenomenological implications of these comparisons are then considered. The bases for comparison are: $p_{T}$ and $\sqrt{s}$ dependence, and relative magnitudes, of cross-sections and $K$ factors. (In comparing different ISAJET samples, gluon splitting and flavor excitation contributions are considered separately; this cannot be done for NDE results, which do not distinguish these higher order processes.) Comments are also made about the correlation plots and their implications for heavy flavor production dynamics.

Results are compared to the perturbative calculation of $\mathrm{NDE}$; this provides information on the reliability of ISAJET estimates of signals and backgrounds for $Q \bar{Q}$ production. The ISAJET estimates exceed the total NDE cross-section by factors of 1.5 to 3 depending on $\sqrt{s}, p_{T, b}$, and the structure functions used. Better agreement is found for the DFLM parton distributions plus two-loop coupling constant, i.e. the same expression used in NDE. We also observe that the ISAJET $K$ factors are larger than those obtained from the NDE total cross-sections at high $p_{T}$ (but lower for $p_{T}<10 \mathrm{GeV}$ ). However, the DFLM parameters yield $K$ factors which are larger than the ratios computed using the EHLQ1 distributions. Such discrepancies may lead to overestimates of backgrounds to bottom quark production rates.

In order to interpret the results, it is necessary to understand how the magnitudes of the parameters used in our samples change from one set to the next. It is useful to present a set of figures for $\alpha_{s}$ and for the EHLQ1 and DFLM distributions 
in order to study the effect of these functions on the cross-sections.

Figure 35 shows the relative size of the coupling strength as a function of $p_{T}$ for all three choices of $\alpha_{s}$. For this plot $m_{b}=4.75 \mathrm{GeV}, Q^{2}=p_{T}^{2}+m^{2}$, and $\Lambda_{Q C D}=$ $260 \mathrm{MeV}$. These are the "modified" parameters used in the DFLM set. Figure 36 is a similar plot for which the appropriate masses and scales for each data sample are used when $\alpha$, is calculated. Figure 37 presents the gluon distribution for EHLQ1 and DFLM as a function of $p_{T}$ at $\sqrt{s}=1800 \mathrm{GeV} \cdot Q^{2}=p_{T}^{2}+m_{b}^{2}$ and $x=2 p_{T} / \sqrt{s}$ in this calculation. Figure 38 does the same for the bottom quark distribution with $p_{T, b}>$ $12 \mathrm{GeV}$.

Figures 35 through 38 give insight into the ISAJET results. For example, Figure 35 indicates that the "intermediate" $\alpha_{s}$ is about 10 percent smaller than "default" $\alpha_{s}$; this is reflected in the "intermediate" cross-section being lower than the "default" cross-section. This decrease is seen in the results at both center-of-mass energies. One might also anticipate that the larger $b$ quark structure function in DFLM (fig. 38) would lead to a larger cross-section for the flavor excitation events; however, the strong coupling strength is reduced in magnitude in the "modified" sample. To estimate the effect of these various choices of parameters, test files were generated in selected bins of transverse momentum for the $b$ quark. The difference in going from the one to two-loop coupling can lead to changes in the cross-section per bin on the order of 40 percent, while varying the mass or the factorization scale or the structure functions never changes the value by more than 20 percent.

At $\sqrt{s}=630 \mathrm{GeV}$ note that the lowest order cross-section is larger than the gluon splitting and flavor excitation cross-sections for all $p_{T}$ for the samples which use the EHLQ distributions, figures 13 and 14. At $1800 \mathrm{GeV}$, these samples show that gluon splitting and flavor excitation are the dominant contributions to the crosssection for $p_{T, \min }>10 \mathrm{GeV}$. At each energy, the "modified" flavor excitation crosssection is larger than that of either "default" or "intermediate" ISAJET. The "mod. ified" lowest order and gluon splitting cross-sections are smaller than those of the other two sets. This difference can be attributed to the smaller gluon distribution 
found in DFLM. The flavor excitation samples have a $b$ quark in the initial state, and the larger heavy flavor component of the higher order DFLM structure functions compensates for the softer glue in such processes as $b+g \rightarrow b+X$.

The inclusive distributions shown in figures 16 and 21 are the sums of the lowest order cross-section with the contributions from gluon splitting and flavor excitation. At both center-of-mass energies the "modified" ISAJET cross-sections are smaller than the "default" values. Although the $b$ quark mass, parton densities, and factorization scale are varied in our three samples, the band bordered by the "default" and "modified" curves in figures 16 and 21 is at best only a rough indication of the theoretical uncertainty associated with the ISAJET cross sections. The full range of uncertainty is no doubt larger. The uncertainty in figures 16 and 21 is about a factor of two for high $p_{T, b}$ at both values of $\sqrt{s}$.

NDE present an "error" band set by $\frac{1}{2}\left(p_{T}^{2}+m_{b}^{2}\right)<Q^{2}<2\left(p_{T}^{2}+m_{b}^{2}\right), 4.5<$ $m_{b}<5 \mathrm{GeV}$, and $160<\Lambda_{4}<360 \mathrm{MeV}$. The "modified" ISAJET cross section falls within this band. However, the central values of the parameters used in the NDE calculation are identical to those of our "modified" ISAJET calculation. Thus, one might expect a priori that the dashed "modified" ISAJET curves in figures 16, 17,21 , and 22 should agree identically with the solid NDE curves. The difference is a quantitative measure of the difference between the analytic and Monte Carlo approaches to computing bottom quark rates at large $p_{T}$.

An important observation is that at both center-of-mass energies, the ISAJET inclusive cross-sections and $K$ factors are larger than the NDE result for $p_{T, b}>15$ $\mathrm{GeV}$. In this $p_{T, b}$ range, the "default" and "intermediate" cross-sections generated with the EHLQ set 1 parton densities are at least twice as large as the NDE values. As shown in figures 16 and 21, at both center-of-mass energies the "modified" curves are about 1.4 times the central NDE value.

At $1.8 \mathrm{TeV}$, all three sets of Monte Carlo results give large $K$ factors, between 4.2 and 5.5 for $p_{T, b}>25 \mathrm{GeV}$, as shown in figure 21. The "modified" curve is 
consistently higher over this range, with values centered around 5.4, while "default" and "intermediate" ISAJET yield values between 4 and 5 . These values are larger than the respective $K$ factors at $630 \mathrm{GeV}$. The "default" and "intermediate" values at $630 \mathrm{GeV}$ (fig. 17) center around 2.3 while the $K$ value from the "modified" set lies between three and four. Note that the Monte Carlo $K$ factors drop steeply to 1 for $p_{T, b}<20 \mathrm{GeV}$ at both center-of-mass energies. This is beculuse of the cut on $p_{T, b}(12$ $\mathrm{GeV}$ ) for the flavor excitation and gluon splitting samples. The analytic calculation has no such artificial cut (the low- $p_{T}$ divergence is absorbed into the parton densities); thus, the analytic $K$ factor is smooth at low values of transverse momentum, as shown in the figures.

Over the relevant $p_{T}$ ranges, NDE calculate a $p_{T}$ dependent $K$ between 2.6 to 3.2 at $1800 \mathrm{GeV}$, and $K$ between 1.8 to 2.4 at $630 \mathrm{GeV}$. At both energies the ISAJET $K$ factors are larger than the NDE result for $p_{T, b}>20 \mathrm{GeV}$. ISAJET therefore overestimates the $O\left(\alpha_{s}^{3}\right)$ higher order contributions to the cross-section. Figures 17 and 22 exhibit the $p_{T, b}$ dependence of $K$ for centrally produced $b$ quarks. For $p_{T}>20$ $\mathrm{GeV}$ at $\sqrt{s}=630 \mathrm{GeV}$ the "modified" $K$ factor decreases from 4 to 3 as $p_{T}$ approaches $40 \mathrm{GeV}$, while the default and intermediate ratios remain fairly constant at 2.6 . Although $K_{\text {modified }}$ is greater than $K_{\text {default }}$, the "modified" inclusive cross-sections (figs. 16 and 21) do not overestimate NDE by factors as much as do the others. One sees better agreement for the cross-sections than the $K$ factors; this is because the lowest order $b \bar{b}$ cross-sections are much smaller for the two-loop parameters for $\alpha_{s}$.

The NDE results show that $K$ is a mildly decreasing function of $p_{T}$ at both energies. Note that the ratio $K$ at $\sqrt{s}=630 \mathrm{GeV}$ in the "modified" case peaks at a $p_{T, b}$ of $20 \mathrm{GeV}$, and then declines as the transverse momentum increases. Test samples of flavor excitation and lowest order $b$ production, with gluon splitting omitted, lead to a curve at this energy which continues to drop to $K=1$ as $p_{T}$ approaches $80 \mathrm{GeV}$. This reflects the fact that the flavor excitation contribution becomes less pronounced in ISAJET at high $p_{T}$. Figures 13 through 15 exhibit this effect at $\sqrt{s}=630 \mathrm{GeV}$. 
At $p_{T, b}=80 \mathrm{GeV}$, the gluon splitting and flavor excitation cross-sections are much smaller than the lowest order contribution. An indication of this decrease is also seen at $\sqrt{s}=1800 \mathrm{GeV}$. Cudell et al. ${ }^{29}$ present $K$ factor calculations for different Monte Carlos for $p_{T, b}$ out to $1000 \mathrm{GeV}$ for a center-of-mass energy of $16 \mathrm{TeV}$. All the computer algorithms eventually show a dropoff in the value of $K$ while the analytic result remains constant.

Higher order contributions to ISAJET heavy flavor cross-sections become larger when the center-of-mass energy is raised from $630 \mathrm{GeV}$ to $1800 \mathrm{GeV}$. This is also true for the NDE calculation. However, the ratio $K_{1800} / K_{630}$ is approximately constant for large $p_{T}$ for any parameter set. The ISAJET overestimate of the NDE $K$ factor can be expressed as a ratio $K_{m o d} / K_{N D E}$. For $p_{T, b}>25 \mathrm{GeV}$, this value also appears to be approximately constant at $\simeq 1.8$; there is little $p_{T, b}$ or $\sqrt{s}$ dependence. Extending these results to higher values of $p_{T}$ and $\sqrt{s}$ would be useful in studies of heavy flavor production at future hadron colliders. Perhaps ISAJET overestimates of the cross-section might be taken into account by a simple correction factor.

One can relate the discrepancy between the ISAJET cross-sections and the analytic result to the ideas discussed in chapter 2 . The high $p_{T, Q}$ gluon radiation effects and the heavy flavor structure function effects are small out to $p_{T, b}<80 \mathrm{GeV}$, so they cannot be responsible for the ISAJET cross-section being larger than the NDE result. The physics leading to Coulomb-type threshold enhancements is not included in the Monte Carlo code used here. This leaves the "non-interference" approximation discussed by R. Meng and colleagues ${ }^{28}$ as the major cause of the difference between the two calculations.

A phenomenology issue to be aware of as a result of this discrepancy is the possibility of incorrectly estimating bottom quark backgrounds to other physical processes of interest. The UA1 collaboration has published results ${ }^{18}$ which indicate that at $\sqrt{s}=630 \mathrm{GeV}$ the experimental cross-sections for bottom quark production are in reasonable agreement with those calculated by NDE (see fig. 16). Preliminary results 
from $\mathrm{CDF}^{19}$ show the $b$ quark cross-section to be larger than the NDE result.

Experimentally, a detailed understanding of bottom (and charm) production is essential for estimating backgrounds to production of heavier objects. To define sensible selection criteria for extracting signal from background, one must understand the typical event topology within the detector-i.e., differential distributions in relevant kinematic variables. While $2 \rightarrow 2$ QCD processes produce back-to-back $b \bar{b}$ pairs, higher order processes produce events with more complex topologies involving an extra hadronic jet. ${ }^{7}$ An experimental handle on higher order topologies is the distribution in the difference $\Delta \phi$ between the azimuthal angles of the $b$ and $\bar{b}$. In lowest order, $b$ and $\vec{b}$ will be back-to-back $\left(\Delta \phi=180^{\circ}\right)$; gluon splitting produces events with $\Delta \phi \simeq 0$, and flavor excitation provides a broad distribution in $\Delta \phi .^{7}$ Other lowest order results-such as low pair transverse momentum $\left|\vec{p}_{T, b}+\vec{p}_{T, \bar{b}}\right|$, and broad positive correlation $^{8}$ of $y_{b}$ and $y_{\bar{b}}$-will also be altered by higher order processes. Data will provide important guidance for refinements of both the analytical and Monte Carlo approaches.

Figures 23 to 26 present the singly differential distributions in transverse momentum and rapidity for the $b$ quark using the selections made for the correlation plots (see table 2). It is interesting to observe that the higher order contributions in the $p_{T, b}$ and in the rapidity distributions have roughly the same shape as the lowest order curves. This behavior favors the assumption that correction factors may perhaps be used to model heavy flavor production in Monte Carlo calculations. This notion is disabused after study of the correlation plots, however, since the three processes have different behavior in the $\Delta y$ and $\Delta \phi$ distributions. It is difficult to see how one can use simple "K-factor" type corrections to treat these correlation distributions.

The analytic calculation of heavy quark correlation distributions is still work in progress. Nevertheless, the ISAJET distributions presented in figures 27 through 34 suggest several qualitative issues to be considered when one eventually makes the comparison of Monte Carlo results with analytic expressions. For example, the 
$\left|\vec{p}_{T, b}+\vec{p}_{T, b}\right|$ plots (figs. 27 and 28 ) provide an effective way to separate higher order effects from the lowt. $t$ order production processes. At least two orders of magnitude separates the gluon split:ing cross-section from the lowest order result at $p_{T}^{\text {pair }}=80$ $\mathrm{GeV}$. Such an analysis would require a thorough understanding of initial and final state gluon radiation; these processes are responsible for the lowest order distribution having non-zero values, as one would expect from the naive parton model. The $p_{T}^{\text {pair }}$ results suggest that studies of transverse momentum correlations could also be useful in tagging higher order processes. Knowledge of the $p_{T, Q}$ distributions, given a selection on $p_{T, Q}$, might help in analyzing backgrounds to top quark production and in studying mixing and $\mathrm{CP}$ violation phenomena.

The ISAJET results suggest that the addition of gluon splitting events to the lowest order sample changes the shape of the total rapidity difference distribution by narrowing it. in width by approximately 1 unit in rapidity. The change of the transverse momentum cut on the $b$ quark from 10 to $20 \mathrm{GeV}$ has the effect of increasing the relative contribution from the gluon splitting and flavor excitation samples in the correlation distributions. This can be seen by studying figure 18 , the integrated crosssection in $p_{T, b}$ for the default sample. For the cut at $10 \mathrm{GeV}$ (fig. 29), the full width of the rapidity distribution at half maximum for the lowest order sample is about 4 units, while the total distribution has a FWHM equal to 2.8. The numbers for $p_{T, b}>$ $20 \mathrm{GeV}$ are similar (fig. 31); FWHM goes from 3.6 to 2.6 when the higher order cross-section is added.

Reference 8 contains a lengthy discussion on the relationship between the lowest order dynamics and the correlation distributions. It appears that several of the arguments and observations presented there can be applied to the ISAJET higher order distributions in order to gain some understanding of the behavior seen in the ISAJET samples. The basic point is that the Born cross-sections are dependent only on $\Delta y$ and favor a broad distribution in that variable, while the parton distribution functions can act to suppress contributions at large $\Delta y$. At lowest order one sees broad correlations due to $g g \rightarrow Q \bar{Q}$. For the higher order effects the gluon splitting 
processes, which are presumed to be primarily due to initial state gluons, lead to a narrowing of the distribution.

More detailed studies of correlation behavior will include plots of $\Delta y$ with different rapidity selections on the $b$ quark. For example, by choosing a cut $1<y_{b}<3$ on the $b$ quark one can check for positive correlation behavior of the partner. Such calculations have already been carried out in the case of lowest order production; they exhibit positive correlation behavior between the quark and the anti-quark. ${ }^{8}$ The $\Delta y$ plots presented in figures 30 and 32 confirm this behavior for the lowest order processes, since the distribution is only slightly shifted in the positive direction with the peak $\Delta y<1$. ("Perfect" positive correlation is marked by the $\Delta y$ distribution remaining centered at zero, i.e. $y_{b}=y_{\bar{b}}$.) The gluon splitting samples exhibit strong positive correlations. One might expect that the properties of the gluon splitting function which govern this mechanism do not depend strongly on the rapidity of the initial gluon. The rapidity difference distribution for the flavor excitation samples are quite broad due to the $\bar{b}$ having large values of $|y|$. The effect of this binodal distribution of the antiquark can be seen in the asymmetric $1<y_{b}<3$ plots.

The ratio of the cross-sections at zero and 180 degrees in the azimuthal angle plots (figs. 33 and 34 ) provides a quantitative measure of the relative contribution of higher order effects. For $p_{T, b}>10 \mathrm{GeV}$, the back to back cross-section is about ten times larger than the cross-section at zero degrees. This ratio is reduced by about half when the transverse momentum cut on the $b$ quark is increased to $20 \mathrm{GeV}$. These numbers are consistent with the cross-sections at $1800 \mathrm{GeV}$ center-of-mass energy shown in table 2. Between $p_{T, b}=10 \mathrm{GeV}$ and $p_{T, b}=20 \mathrm{GeV}$ the flavor excitation and gluon splitting cross-sections become larger than the lowest order result.

A curious feature of the azimuthal angle distributions is the apparent dip in the cross-section at $\Delta \phi=0$ seen in figure 33. This may be due to the lower bound on $Q^{2}$ imposed by ISAJET on the gluon radiation in the code's evolution algorithm; a gluon that converts into a quark anti-quark pair is required to have some minimum 
energy. This leads to a non-zero opening angle between the $Q$ and $\bar{Q}$.

The correlation plots presented here allow rough estimates of the relationship between the correlation variables themselves. A cut on $|\Delta y|<1$ would tend to favor higher order contributions to the azimuthal angle distributions. Selecting $|\Delta y|>2$ would tend to eliminate most of the gluon splitting events and cause a drop-off in the azimuthal angle distribution for small angles. This behavior has been seen in Monte Carlo calculations of $\Delta y$ vs. $\Delta \phi$ performed by Webber and Ellis. ${ }^{34}$ Similarly, a selection on $p_{T, p a i r}$ could be used to negate lowest order production. The $\Delta \phi$ distribution would lose the peak at 180 degrees. Cuts on $\Delta \phi$ may also be employed to study $p_{T}$ and rapidity distributions of lowest order and higher order events. Perhaps the most challenging study would be to find some set of selections which would tend to favor the flavor excitation contribution. This might be of interest to someone wishing to study heavy flavor parton distribution functions. The plots presented here indicate that some combination of cuts on azimuthal angle (restricted to about 120 degrees) and rapidity difference (taking advantage of the strong positive correlations seen in the gluon splitting events) might help in this regard. Correlation distributions in $p_{T, b}$ versus $p_{T, \bar{b}}$ may also be useful.

One can interpret the results presented here in light of proposed experiments to study $B$ meson mixing. The problem is to find the location of the $\bar{B}$ meson given the location of the $B$. At large transverse momentum one can assume that the location of the precursor bottom quarks will represent the position of the resulting leptons seen in the detector. The $\Delta y$ cross-sections indicate that the antiflavor decay will most likely fall within a reasonably narrow rapidity range around the $b$ quark as long as kinematic selections on the events do not cut out the higher order events. The $p_{T}^{\text {pair }}$ distributions suggest that many of the event pairs will have non-negligible transverse momentum in a plane perpendicular to the beam axis. One should also notice that overly stringent selections on the azimuthal angle may eliminate many $B \bar{B}$ events that are generated by higher order processes. 
It has already been established that ISAJET overestimates the cross-section for high transverse momentum bottom quarks. A natural issue is the location of the extra cross-section occurring in the more detailed correlation curve (at high or low rapidity, for example). Studies of this issue will require even larger samples in order to obtain accurate scatter plots relating $p_{T, b}$ to $\Delta y$ and $\Delta . \phi$. The $O\left(\alpha_{s}^{3}\right)$ calculation begins to break down at very large transverse momentum. 'The above mentioned scatter plot studies should help to establish similar problem areas in the exact calculation of the correlation distributions. Future analytical work may also provide an understanding of $b \bar{b}$ threshold effects on correlations. The extension of the analysis done in reference 28 to include differential distributions in transverse momentum, rapidity, and the correlation variables is expected to shed light on these issues. 


\section{CHAPTER 5}

\section{CONCLUSIONS}

Recent developments in the analytic calculation and in the measurement of heavy flavor cross-sections have led to a re-examination of the high $p_{T}$ behavior of heavy flavor production. The calculation of the heavy flavor production cross-section to $O\left(\alpha_{s}^{3}\right)$ in perturbation theory ${ }^{9,10,11}$ provides a new benchmark for higher order calculations and precision tests of QCD. One application of these results is to study the differences between the analytic and Monte Carlo approaches to calculating heavy flavor production cross-sections.

In this work bottom quark production events as simulated by ISAJET have been generated and analyzed. Using three different sets of input parameters, the effects of variations in these parameters have been examined. The reduction of the strong coupling strength $\alpha_{s}$ from the lowest order expression to the two-loop expression is the primary factor determining the relative sizes of the computed cross-sections. Other aspects of the results can be understood from the differences in the gluon and bottom quark structure functions of EHLQ1 and DFLM.

Cross-sections from the ISAJET samples are compared with the next-to-leading order calculation of Nason, Dawson, and Ellis. All ISAJET samples yield a distribution in transverse momentum whose shape agrees with analytic results for $p_{T, b}>15$ $\mathrm{GeV}$. However, ISAJET results consistently exceed the total analytical cross section at large $p_{T, b}$. This result is independent of input parameters and parton distributions (DFLM vs. EHLQ). ISAJET cross sections at $\sqrt{s}=630 \mathrm{GeV}$ are consistent with $\mathrm{UA1}$ data out to $p_{T, b}=20 \mathrm{GeV}$ and preliminary CDF results are more in agreement with ISAJET than with the analytical calculation. 
The $K$ factors from the ISAJET studies are larger than those determined by NDE for large values of transverse momentum. While the parameters of the "modified" ISAJET program yield cross sections closest to those of NDE, they yield $K$ factors furthest from NDE's. This discrepancy has consequences for experimental tests of QCD, and for background estimates involving heavy flavor production and other new physics. The large higher order contributions to the cross section become most apparent in correlation studies of heavy flavor pairs. These effects are seen, for example, in the narrowing of the rapidity distribution for $\Delta y_{b \bar{b}}$, and in the increase in cross-section near $\Delta \phi_{b \bar{b}}=0$ due to contributions from gluon splitting. Data on azimuthal angle dependence, $\Delta \phi_{b \bar{b}}$, should be particularly informative. ${ }^{7}$ ISAJET calculations of the distribution in $\Delta \phi_{b b}$, or the transverse momentum of the $b \vec{b}$ pair, should be compared with analytic results as these become available. Such comparisons will be valuable in further assessments of the reliability of Monte Carlo calculations.

Similar Monte Carlo calculations can be made using the parton level program PAPAGENO. Here the exact tree-level $2 \rightarrow 3$ matrix element is included. Depending on kinematics one can generate events which look like either gluon splitting or flavor excitation. The main concern with the PAPAGENO results is how well the tree-level cross-sections do in reproducing the full result. Galtieri et $a l^{35}$ have studied these distributions and have compared them to ISAJET and NDE cross-sections with an eye towards application to CDF physics. Such studies may lead to general conclusions about the relative merits of matrix element versus shower codes in performing Monte Carlo calculations of heavy flavor production rates. Mention should also be made of Monte Carlo codes which attempt to include subtle QCD effects in their radiation algorithm. PYTHIA ${ }^{36}$ uses the Lund algorithm for hadronization and uses a more sophisticated evolution code for gluon radiation which includes coherence effects. These effects are typically small but may play a role in certain kinematic distributions, such as those which involve $\Delta \phi_{Q \bar{Q}}$, the azimuthal angle between the heavy quarks.

Different physics is represented to differing degrees of accuracy in the analytic and Monte Carlo approaches. In the analytic approach, interference effects are 
incorporated at the level of amplitudes, and infrared and collinear divergences are treated properly. However, limitations are associated with the fact that the analytic calculation is done to a finite order in perturbation theory. These limitations reduce confidence in the analytical calculation when $p_{T} \gg m_{b}$. In this region, higher order multiple gluon radiation modifies the $p_{T}$ distribution. Moreover, for $p_{T} \gg m_{b}$, it is appealing to consider that a portion of the cross section is attributable to "flavor excitation". The analytic calculation includes this portion only to $O\left(\alpha_{s}^{3}\right)$ whereas a larger, or, at least, different answer would be obtained by treating the $b$ quark as a parton from the initial hadron. The $b$ quark parton distribution function is, in principle, a quantity which incorporates all orders in $\alpha_{s}$. Comments have already been made on the adjustments for double-counting which must be made if an initial $b$ quark flavor excitation contribution is to be added to the analytic result. This discussion suggests that the fully analytic $O\left(\alpha_{s}^{3}\right)$ approach is preferable for $p_{T} \lesssim$. a few times $m_{b}$. In this domain, the event topology should also resemble that of the lowest order cross sections. However, for $p_{T} \gg m_{b}$, there are good reasons to appeal to the multiple gluon radiation and flavor excitation contributions incorporated in the Monte Carlo approach. How accurately these are included is a matter for further study. It would be valuable to understand how well Monte Carlo simulations may handle the $\ln \left(p_{T} / m_{b}\right)$ behavior which causes the breakdown of the perturbative expansion for $p_{T} \gg m_{b}$. Second, an algorithm should be devised to resolve the double-counting problem of initial-state heavy quarks.

Future research with Monte Carlo techniques might study ISAJET crosssections of very massive ton quarks in kinematic regimes where the higher order corrections are expected to be small. Such samples may prove to be useful "controls" in attempting to sift out the effects of the interference terms in the exact calculation from the flavor excitation and gluon radiation effects discussed in section 2. Valuable work has already been done by Meng et al. in attempting to pin down the source of the Monte Carlo overestimate. ${ }^{28}$ Their analytic results suggest that this overestimate is intrinsic to any Monte Carlo calculation which neglects interference 
effects. Simple correction factors may be employed to compensate for the Monte Carlo overestimates. However, their approximate formulae for initial state radiation differ from the approach used in the ISAJET calculation; the question is whether a correction factor can be successfully incorporated into the Sjöstran 1 algorithm. Even if this could be done, careful studies will be needed to understanc the effects of such corrections on the correlation distributions.

Given the issues presented above, one is led to ask which approach is preferred for comparison to experimental data. The resul's from this work favor the continuing use of analytic calculations, at least at current collider energy and kinematic regimes. The calculations presented in section 2 indicate that the effects due to gluon radiation at high transverse momentum and the neglect of flavor excitation are small out to $p_{T, b}=80 \mathrm{GeV}$ at collider energies of 630 and $1800 \mathrm{GeV}$, while the ISAJET results exhibit an overestimate in these regions. It will be important to understand the kinematic regions where the perturbative calculation becomes unreliable and Monte Carlo approximations are preferred.

An understanding of bottom quark production in leading and next-to-leading order is important, both in its own right and as a background to new physics. The present study addresses some questions, and raises still others; further investigation on a number of fronts is clearly needed. It would be interesting to explore whether changes could be made in ISAJET and in other Monte Carlo simulation programs which would allow them to more accurately reproduce the high $p_{T}$ behavior of heavy flavor production. Such research will probably depend heavily on an understanding of initial state radiation in heavy flavor production and on a deeper understanding of gluon and heavy quark parton distributions at small $x$. The other major line of attack should be to achieve a detailed knowledge of the correlation behavior for both Monte Carlo and analytic calculations. Not only would this lead to a better understanding of the relative merits of the two approaches, but also to a telling probe of some of the actual dynamics of heavy quark production. 
TABLE 1

\section{ISAJET EVENT SAMPLES}

range of $p_{T}^{\text {part }}, \sigma_{\text {total }}$, and number of events passing rapidity cut

\begin{tabular}{|c|c|c|c|c|}
\hline & & default & intermediate & modified \\
\hline \multirow[t]{12}{*}{$630 \mathrm{GeV}$} & lowest & $2-160 \mathrm{GeV}$ & $2-160 \mathrm{GeV}$ & $2-160 \mathrm{GeV}$ \\
\hline & order & $4.35 \mu b$ & $4.60 \mu b$ & $3.59 \mu b$ \\
\hline & & $75000 / 79000$ & $70000 / 79000$ & $76000 / 79000$ \\
\hline & gluon & $12-160 \mathrm{GeV}$ & $12-160 \mathrm{GeV}$ & $12-160 \mathrm{GeV}$ \\
\hline & splitting & $0.366 \mu b$ & $0.328 \mu b$ & $0.238 \mu b$ \\
\hline & 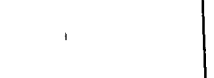 & $52000 / 74000$ & $34000 / 37000$ & $23000 / 37000$ \\
\hline & & & {$[500$ events $/ 2 \mathrm{GeV}]$} & {$[500$ events $/ 2 \mathrm{GeV}]$} \\
\hline & flavor & $12-150 \mathrm{GeV}$ & $12-138 \mathrm{GeV}$ & $12-144 \mathrm{GeV}$ \\
\hline & excitation & $0.154 \mu b$ & $0.136 \mu b$ & $0.234 \mu b$ \\
\hline & & $63000 / 69000$ & $57000 / 63000$ & $30000 / 33000$ \\
\hline & & & & {$\left[500\right.$ events $\left./ 2 \mathrm{Ge}^{r}\right]$} \\
\hline & total & $4.87 \mu b$ & $5.06 \mu b$ & $4.06 \mu b$ \\
\hline \multirow[t]{10}{*}{$1800 \mathrm{GeV}$} & lowest & $2-150 \mathrm{GeV}$ & $2-150 \mathrm{GeV}$ & $2-150 \mathrm{GeV}$ \\
\hline & order & $9.00 \mu b$ & $8.55 \mu b$ & $6.96 \mu b$ \\
\hline & & $49000 / 74000$ & $49000 / 74000$ & $50000 / 74000$ \\
\hline & gluon & $12-148 \mathrm{GeV}$ & $12-144 \mathrm{GeV}$ & $12-150 \mathrm{GeV}$ \\
\hline & splitting & $1.82 \mu b$ & $1.50 \mu b$ & $1.17 \mu b$ \\
\hline & & $42000 / 68000$ & $40000 / 66000$ & $42000 / 69000$ \\
\hline & flavor & $12-150 \mathrm{GeV}$ & $12-150 \mathrm{GeV}$ & $12-150 \mathrm{GeV}$ \\
\hline & excitation & $0.859 \mu b$ & $0.710 \mu b$ & $1.01 \mu b$ \\
\hline & & $39000 / 69000$ & $39000 / 69000$ & $31000 / 69000$ \\
\hline & total & $11.7 \mu b$ & $10.8 \mu b$ & $9.14 \mu b$ \\
\hline
\end{tabular}


TABLE 2

\section{CORRELATION SAMPLES}

number of events, cross-section

\begin{tabular}{|c|c|c|c|}
\hline$\sqrt{s}=1800 \mathrm{GeV}$ & & $p_{T, b}>10 \mathrm{GeV}$ & $p_{T, b}>20 \mathrm{GeV}$ \\
\hline \multirow[t]{7}{*}{$-1<y_{b}<1$} & lowest & 46000 events & 43000 \\
\hline & order & $0.96 \mu b$ & $0.091 \mu b$ \\
\hline & gluon & 32000 & 24000 \\
\hline & splitting & $0.72 \mu b$ & $0.134 \mu b$ \\
\hline & flavor & 31000 & 26000 \\
\hline & excitation & $0.52 \mu b$ & $0.130 \mu b$ \\
\hline & total & $2.20 \mu b$ & $0.355 \mu b$ \\
\hline \multirow[t]{7}{*}{$1<y_{b}<3$} & lowest & 11000 & 9000 \\
\hline & order & $0.59 \mu b$ & $0.045 \mu b$ \\
\hline & gluon & 9000 & 6000 \\
\hline & splitting & $0.42 \mu b$ & $0.041 \mu b$ \\
\hline & flavor & 7000 & 3000 \\
\hline & excitation & $0.26 \mu b$ & $0.050 \mu b$ \\
\hline & total & $1.27 \mu b$ & $0.136 \mu b$ \\
\hline
\end{tabular}




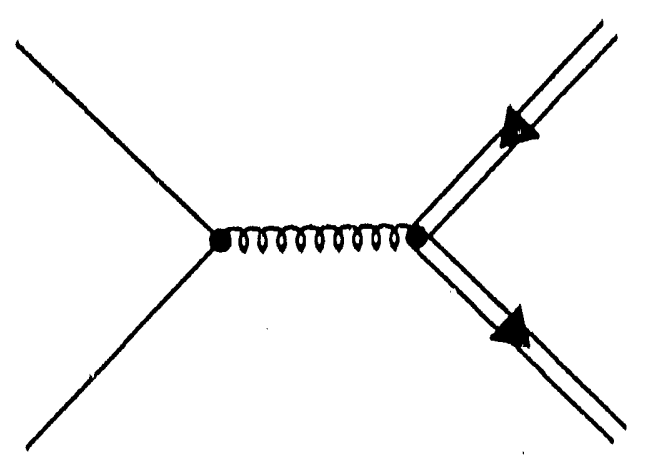

(a)

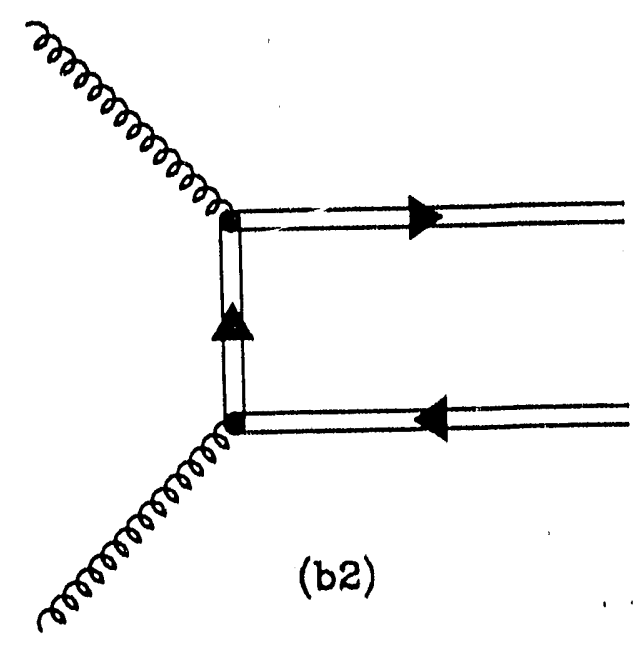

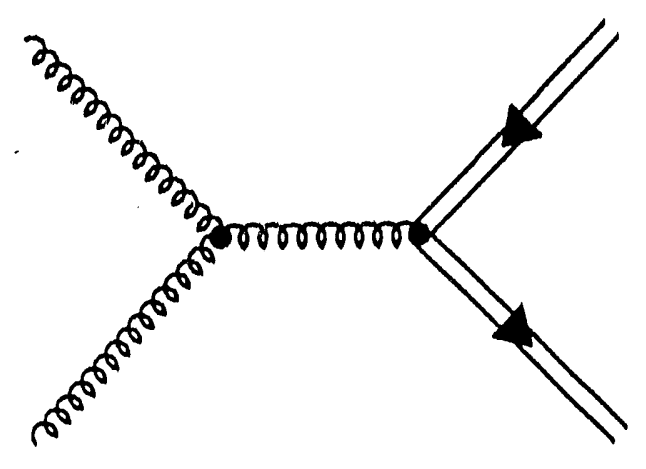

(b1)

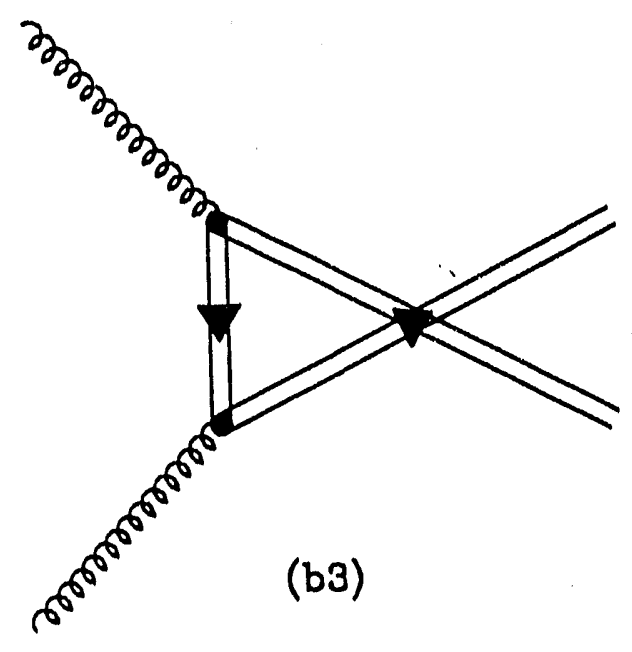

Figure 1 - Lowest order Feynman diagrams for heavy quark production. (a) represents the quark anti-quark annihilation subprocess. $(\mathrm{b} 1,2,3)$ are the gluon initiated subprocesses. (b2) and (b3) involve $\hat{t}$-channel exchange of the heavy flavor. 

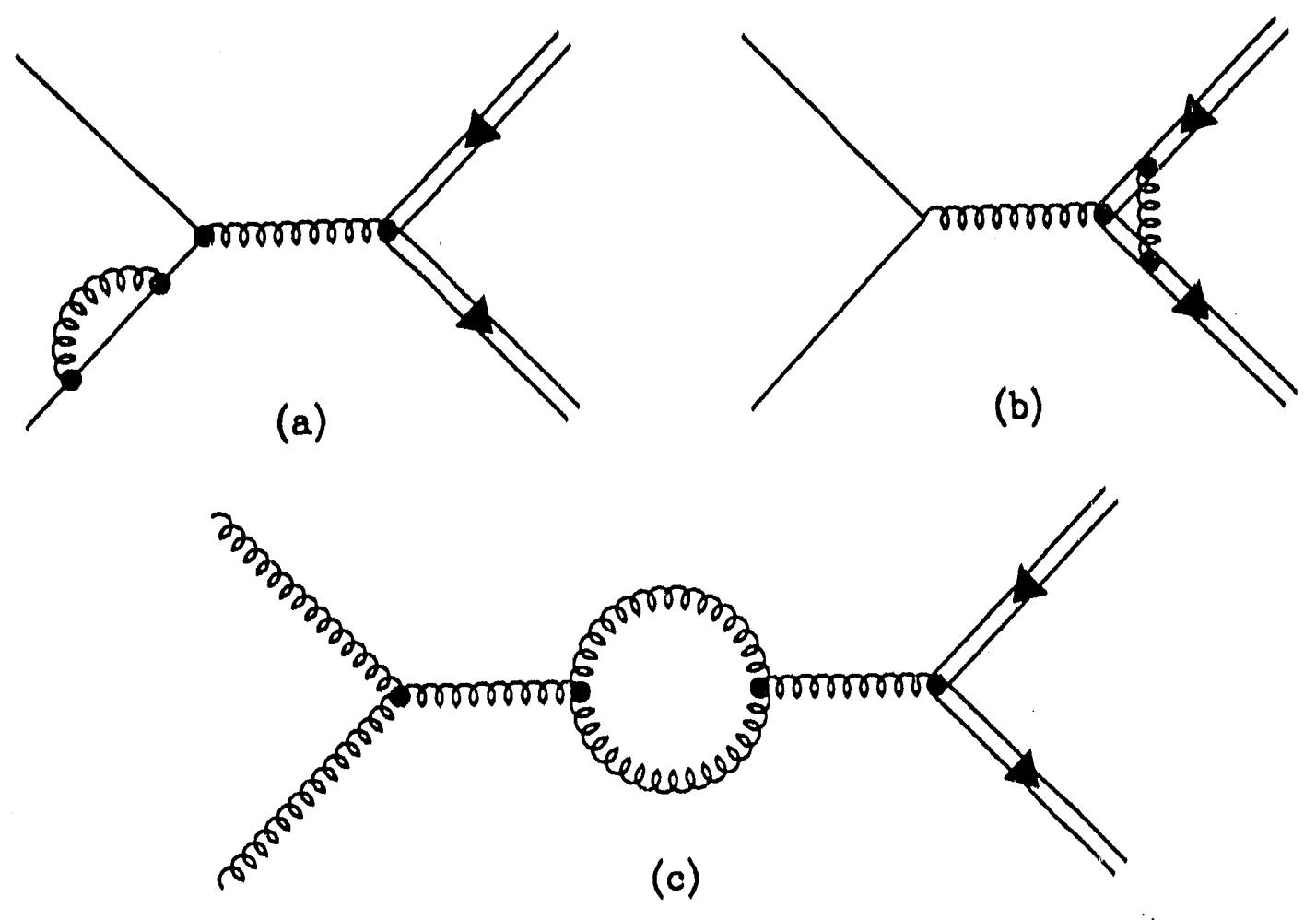

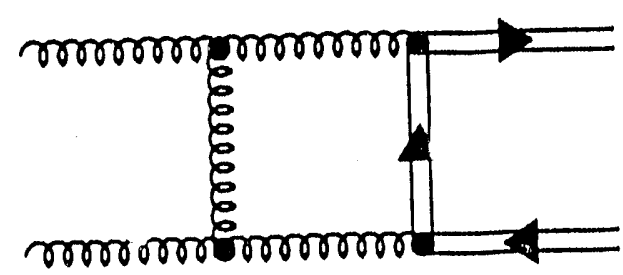

(d)

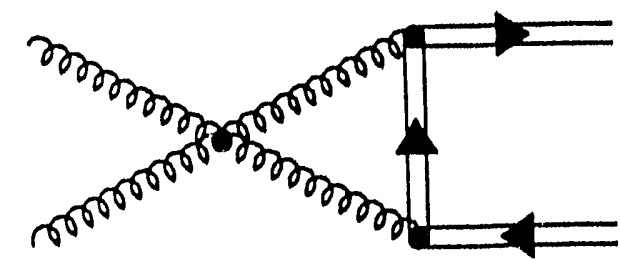

(e)

Figure 2 - Typical higher order diagrams involving virtual corrections to the lowest order diagrams. These diagrams give $\mathrm{O}\left(\alpha_{s}^{3}\right)$ contributions to the cross-section when multiplied with the lowest order diagrams in figure 1. 


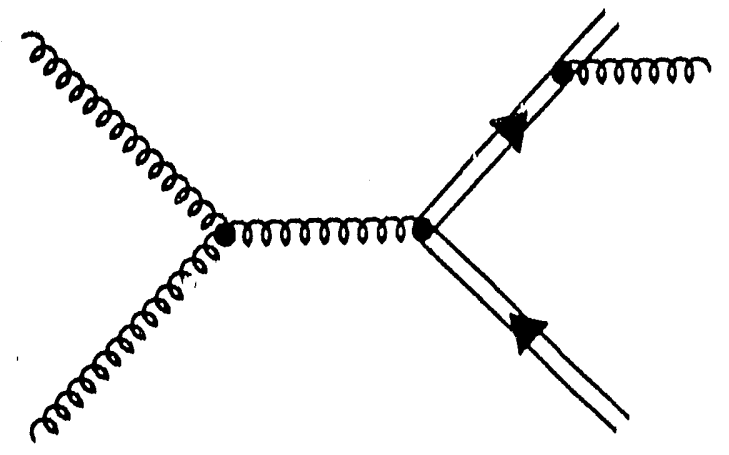

(a)
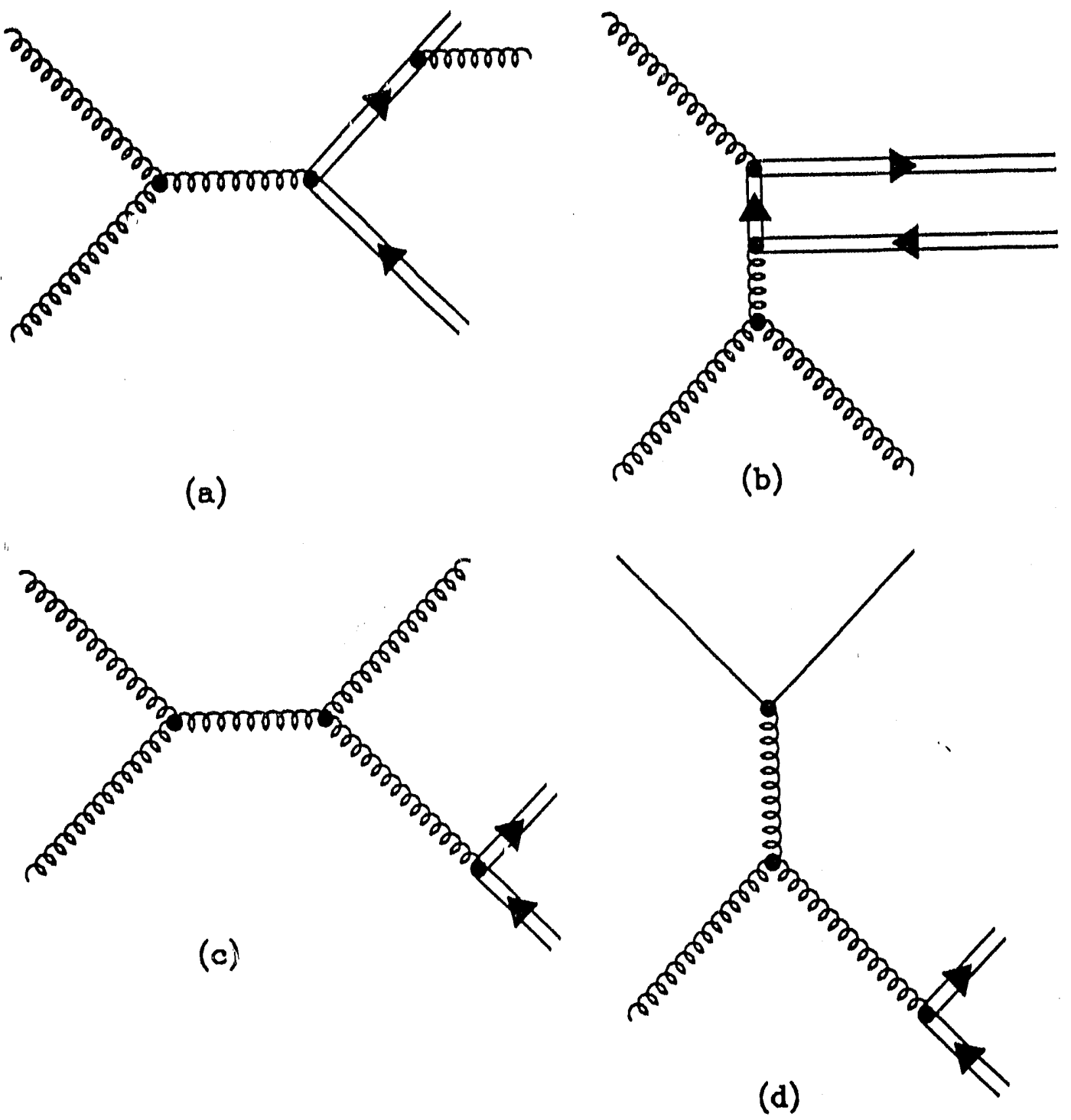

Figure 3 - Higher order tree level processes involving either gluon emission (a) or gluon splitting $(b, c, d)$. 

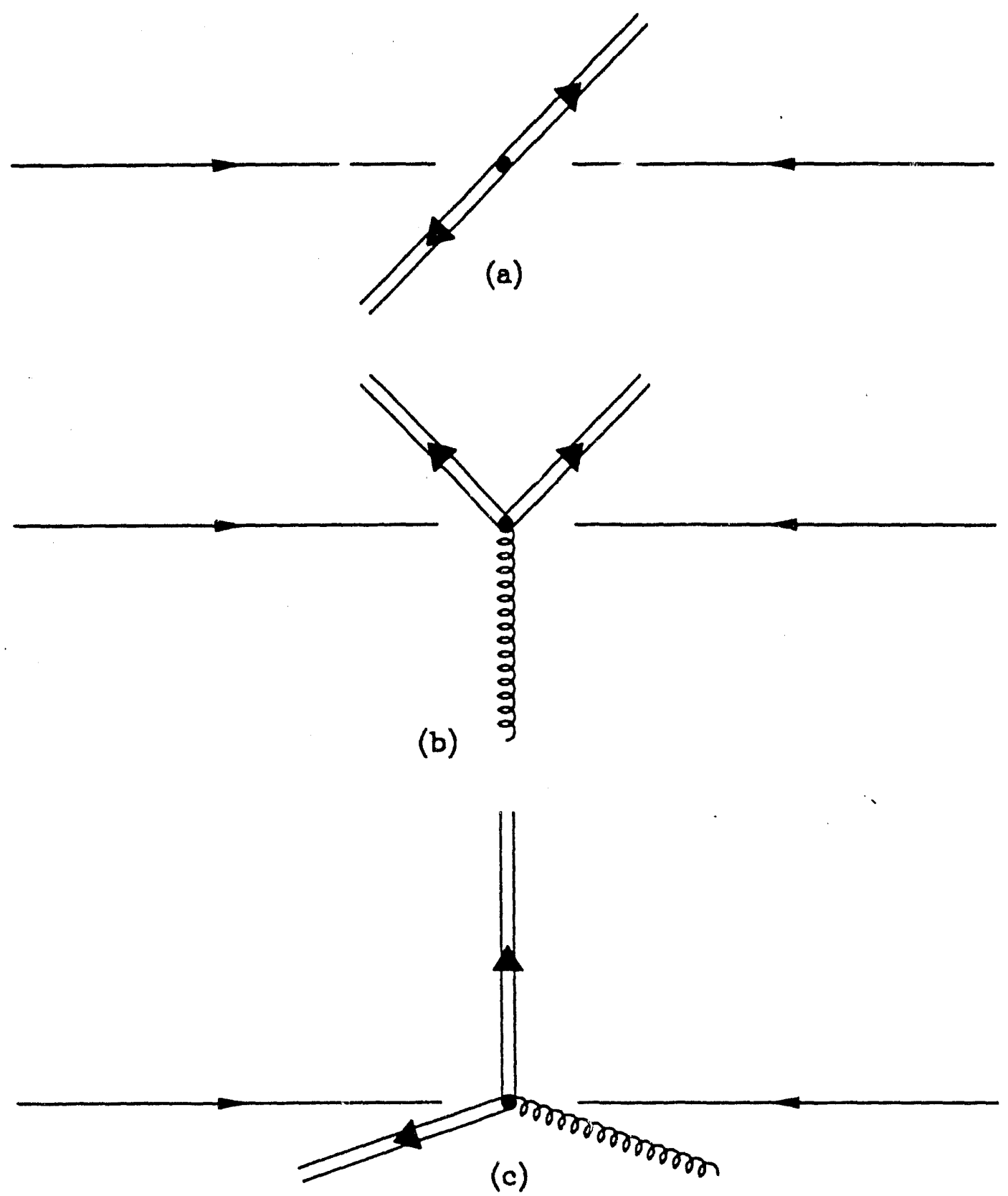

Figure 4 - Event topologies for lowest order and higner order processes. (a) represents lowest order production in which the $Q \bar{Q}$ pair is produced back to back in the parton center of mass frame. (b) and (c) are event topologies which are allowed in higher order processes. In (b) the quark and the anti-quark are produced close together in phase space with momentum balanced by a third jet. (c) represents a piviess in which the heavy guark alone is scattered with large transverse momentum. 


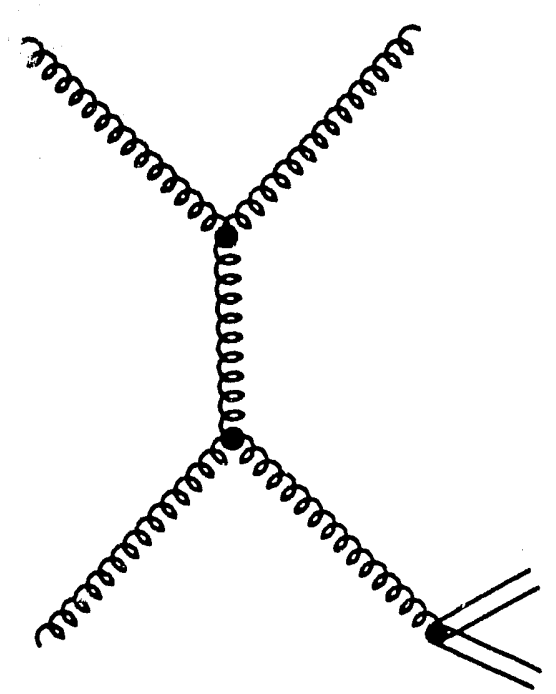

(a1)

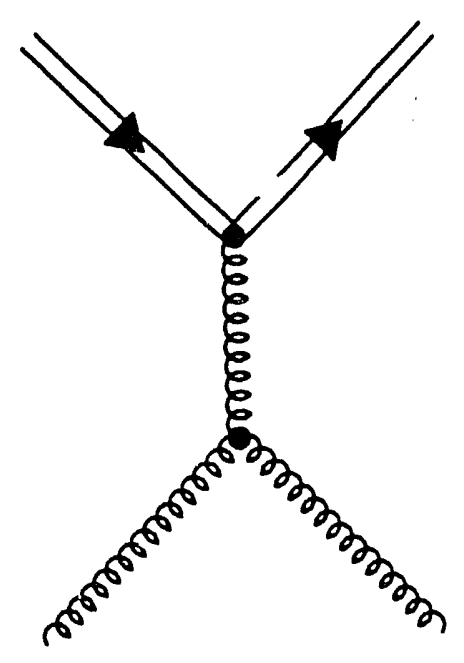

(b1)

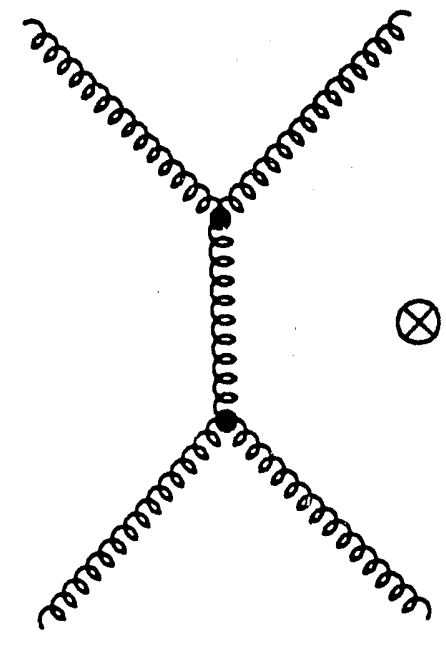

(a2)

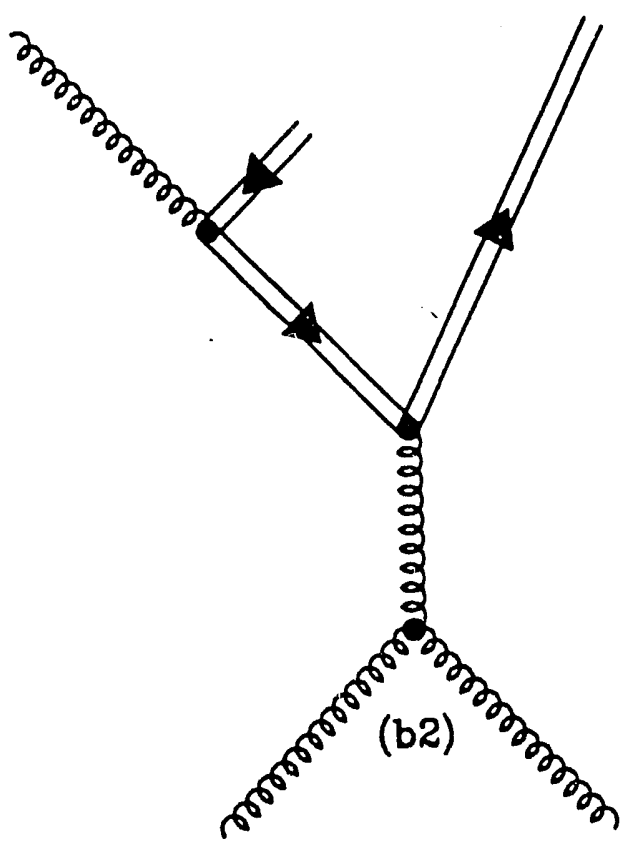

Figure 5 - Diagrams representing gluon splitting events $(\mathrm{a} 1, \mathrm{a} 2)$ and flavor excitation $(\mathrm{b} 1, \mathrm{~b} 2)$. The analytic calculation computes (a1) exactly while the ISAJET Monte Carlo uses a gluon splitting function (a2). Flavor excitation events are calculated in ISAJET by using heavy quark distribution functions (b1) while the analytic calculation uses initial state gluons (b2). 


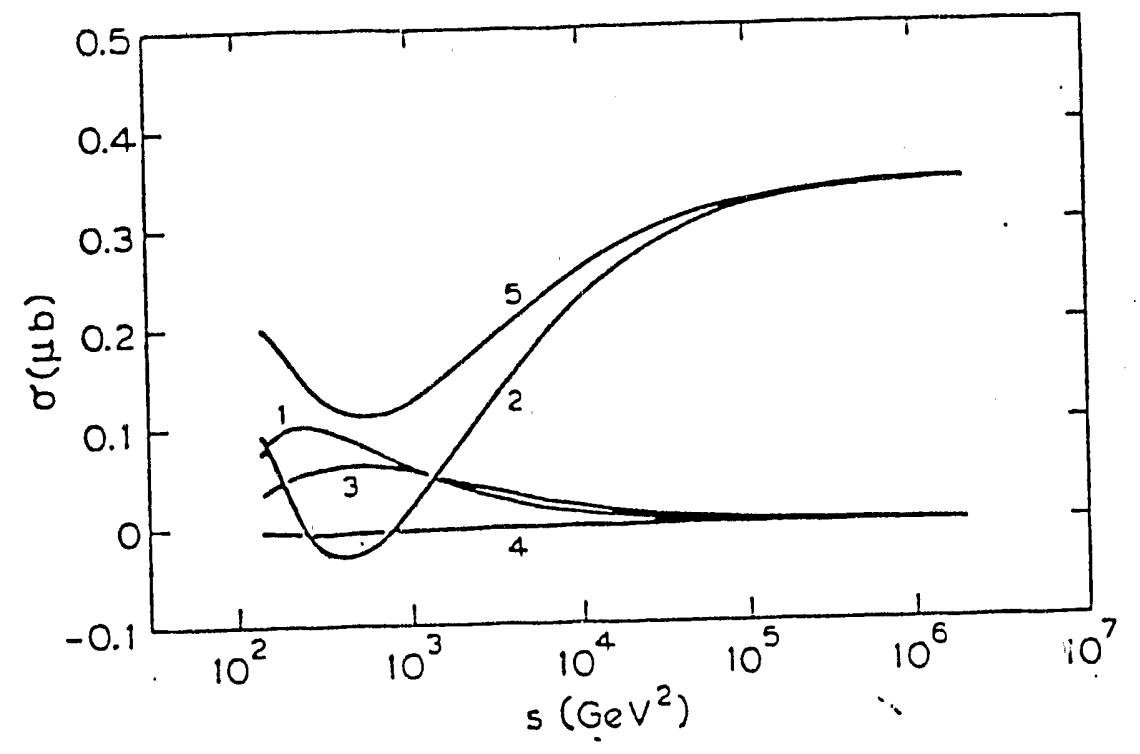

(a)

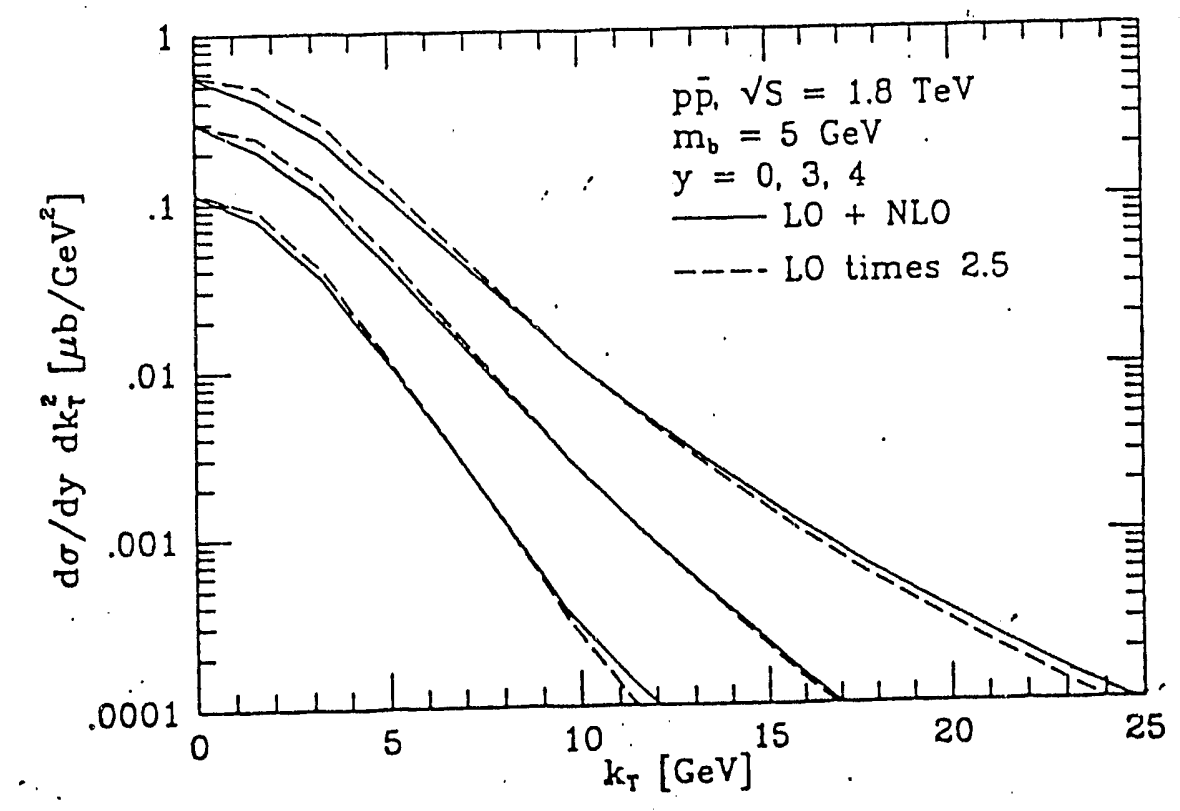

(b)

Figure 6 - Results of $O\left(\alpha_{s}^{3}\right)$ analytic calculations. (a) Beenakker et al. results showing the relative contributions to the total cross-section for $b$ quark production from lowest order (1) and higher order processes as a function of $s$, the parton center of mass energy. Line (5) is the total. (b) Nason, Dawson, and Ellis results showing the dependence of their higher order cross-section as a function of $p_{T, b}$ at $1800 \mathrm{GeV}$. 


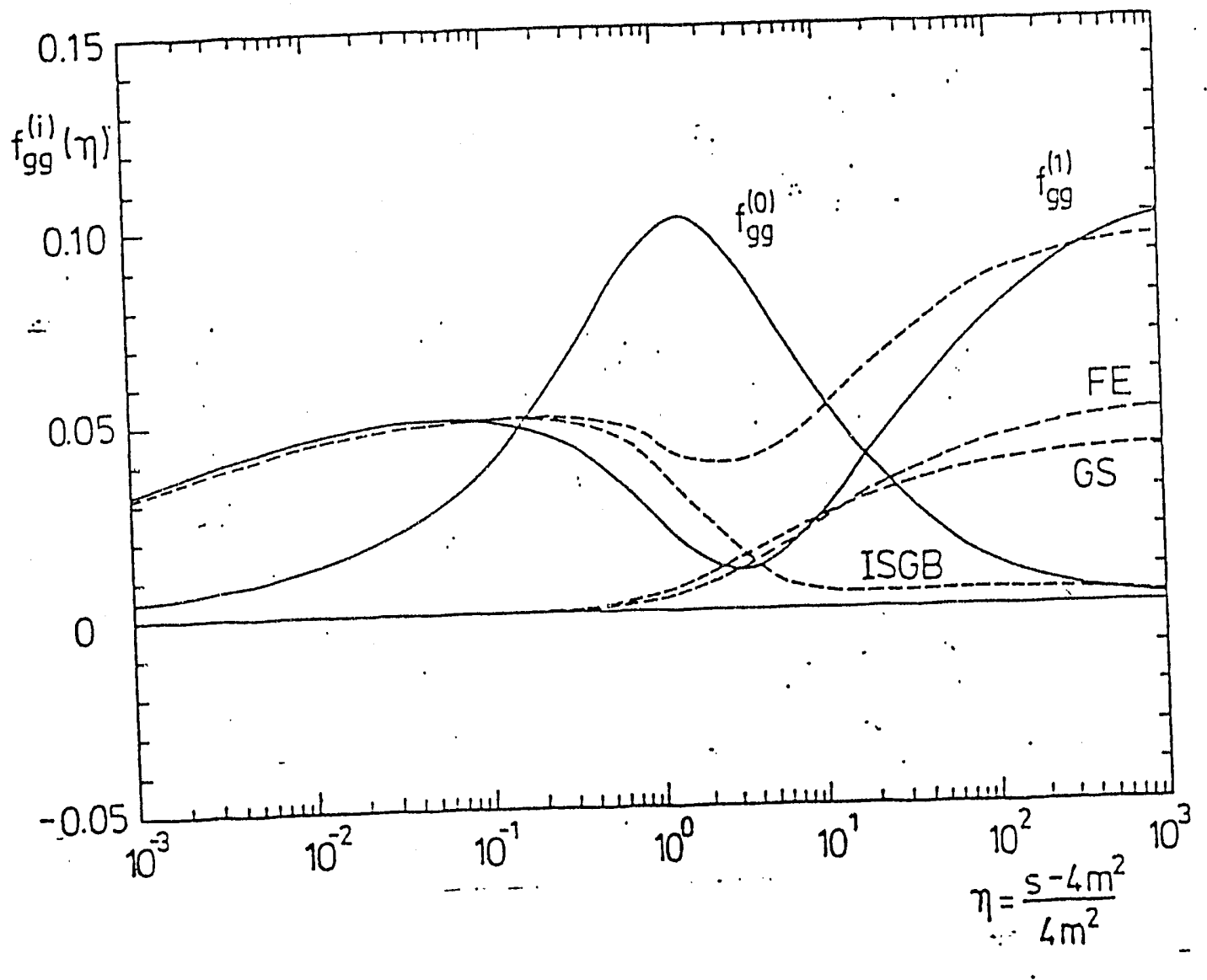

Figure 7 - Results from reference 28 showing the relative contributions to the $g g \rightarrow Q X$ parton level cross-section from lowest order $\left(f_{g g}^{(0)}\right)$ and higher order $\left(f_{g g}^{(1)}\right)$ terms (solid lines). Also shown (dashes) are the results from approximations representing initial state gluon bremsstralung (ISGB), flavor excitation (FE), and gluon splitting (GS) contributions and their sum. $\eta=\frac{3-4 m_{b}^{2}}{4 m_{b}^{2}}$. 


\section{Default ISAJET Lowest Order}

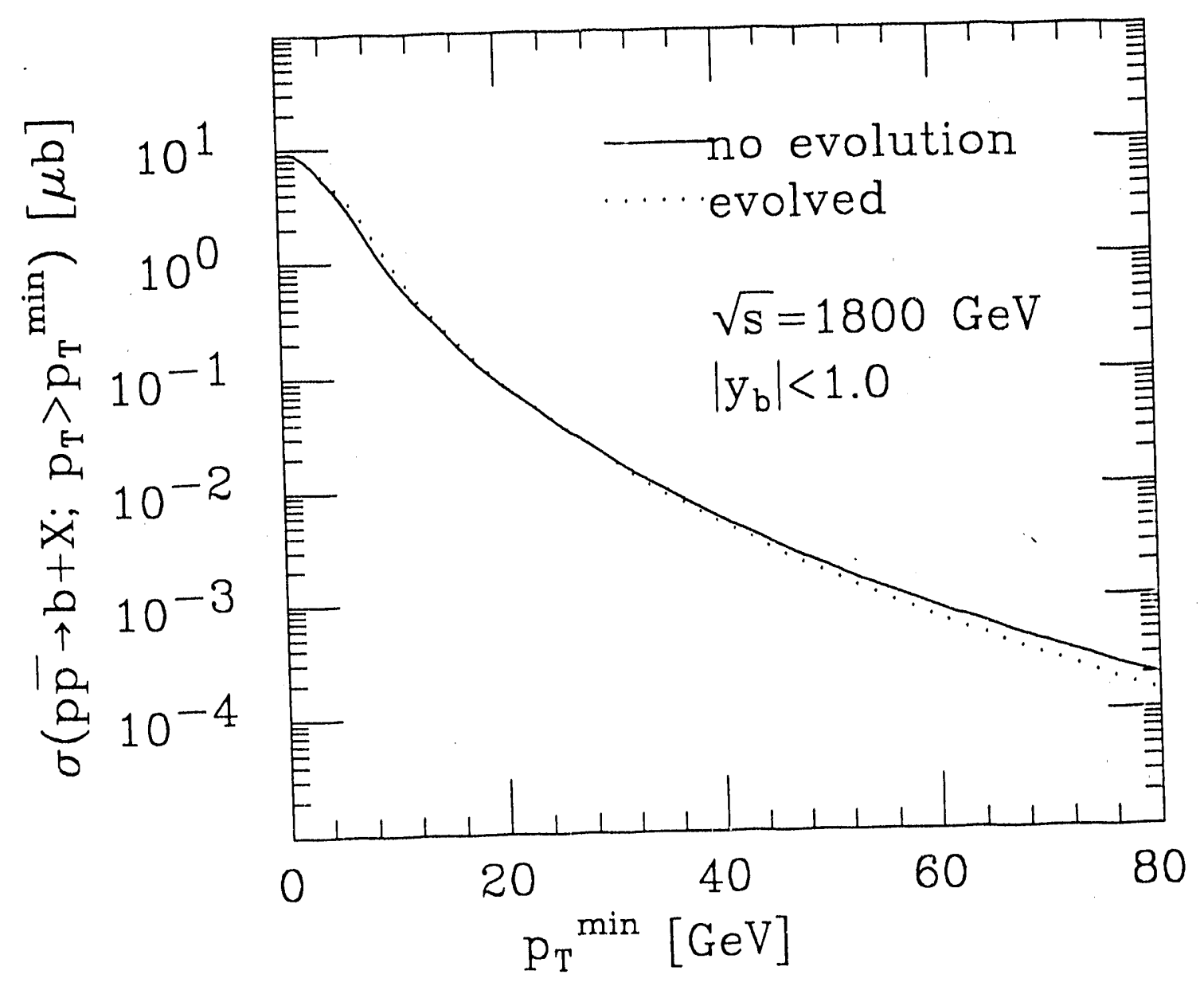

Figure 8 - Lowest order ISAJET cross-section with and without gluon radiation. 
Default ISAJET Flav. Ex.

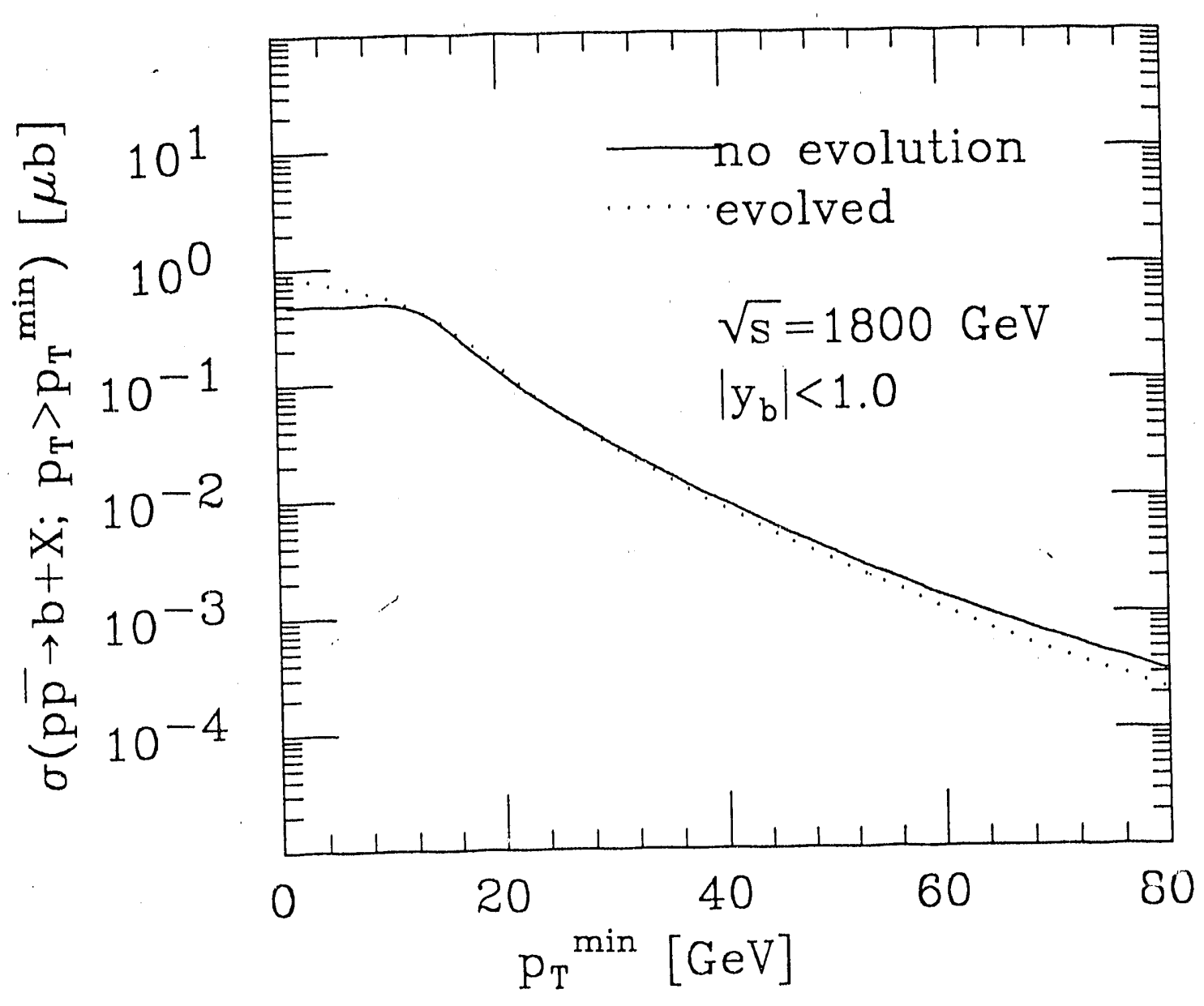

Figure 9 - Flavor excitation ISAJET cross-section with and without gluon radiation. 


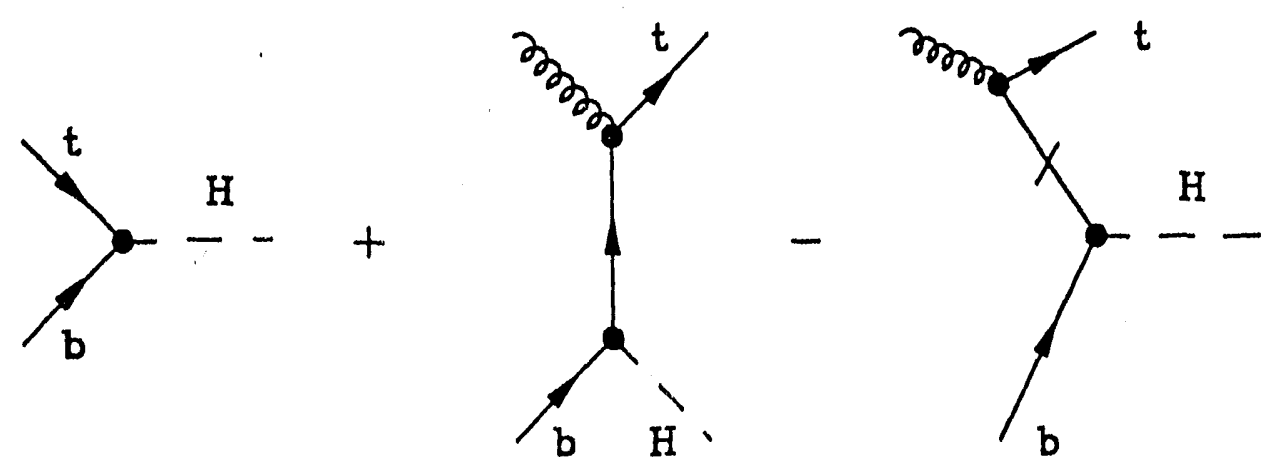

(a)
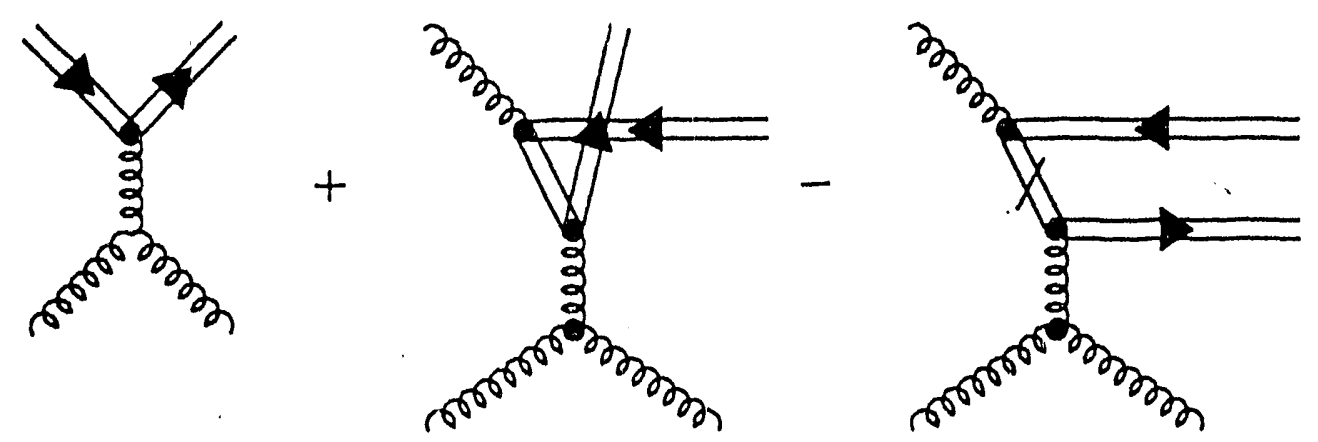

(b)

Figure 10 - Treatment of hard scattering events with a heavy flavor in the initial state. (a) represents $t \bar{b} \rightarrow$ Higgs calculation described in reference 31 . (b) represents a similar subtraction procedure for the $b$ quark. 


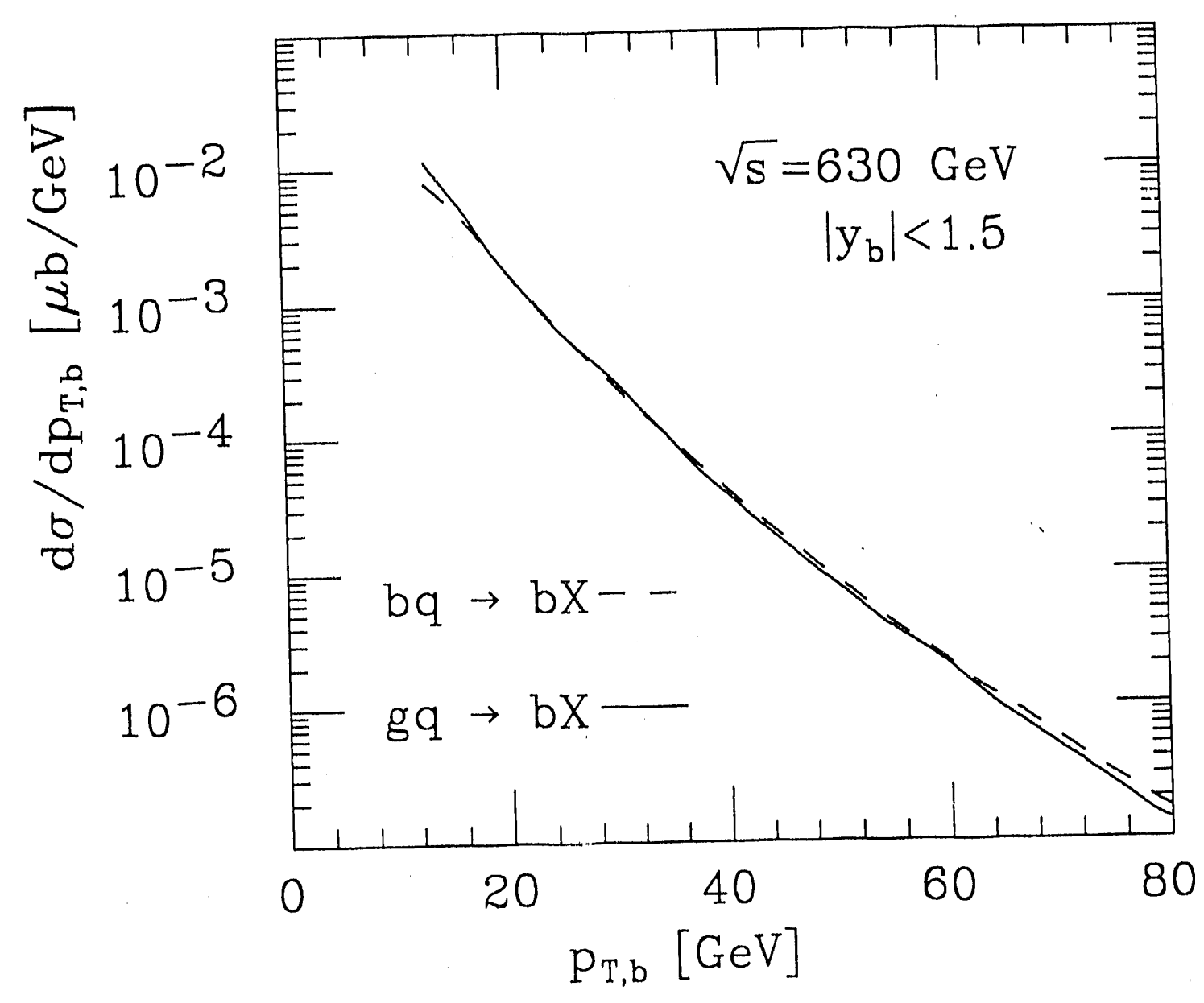

Figure 11 - Differential cross-sections for the processes $b q \rightarrow b X$ and $g q \rightarrow b X$ at $\sqrt{s}=630 \mathrm{GeV}$ (computed using C.P. Yuan's parton level Monte Carlo). 


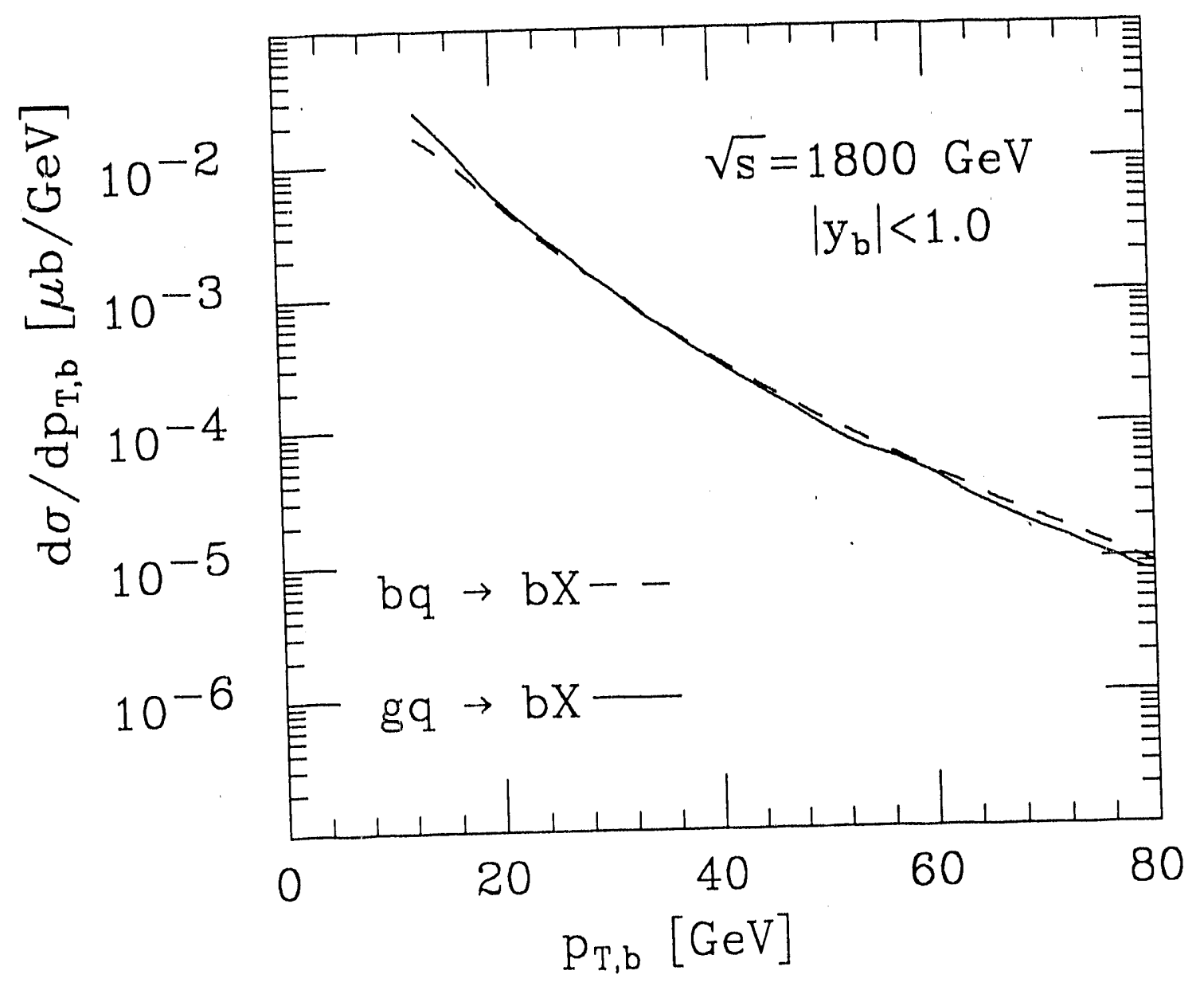

Figure 12 - Differential cross-sections for the processes $b q \rightarrow b X$ and $g q \rightarrow b X$ at $\sqrt{s}=1800 \mathrm{GeV}$ (computed using C.P. Yuan's parton level Monte Carlo). 


\section{Default ISAJET}

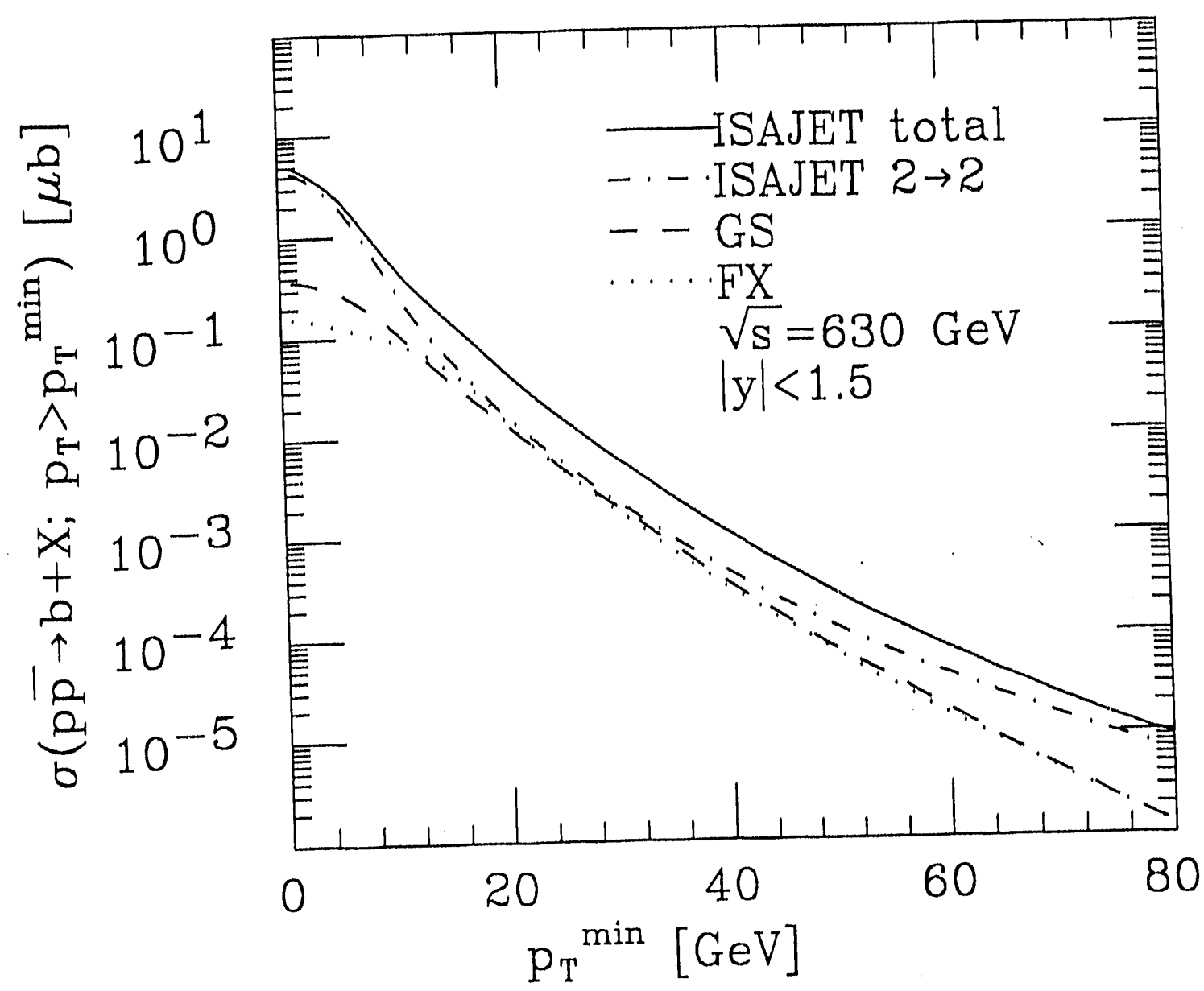

Figure 13 - Cross-sections from "default" ISAJET at $\sqrt{s}=630 \mathrm{GeV}, \sigma(p \bar{p} \rightarrow$ $\left.b+X ; p_{T, b}>p_{T, \min }\right)$ is plotted against $p_{T, \min }$. The rapidity cut on the $b$ quark is $\left|y_{b}\right|<1.5$ at this energy. The solid line is the total inclusive cross-section. Here and in the diagrams that follow, "FX" refers to the flavor excitation sample and "GS" refers to the gluon splitting sample. 


\section{Intermediate ISAJET}

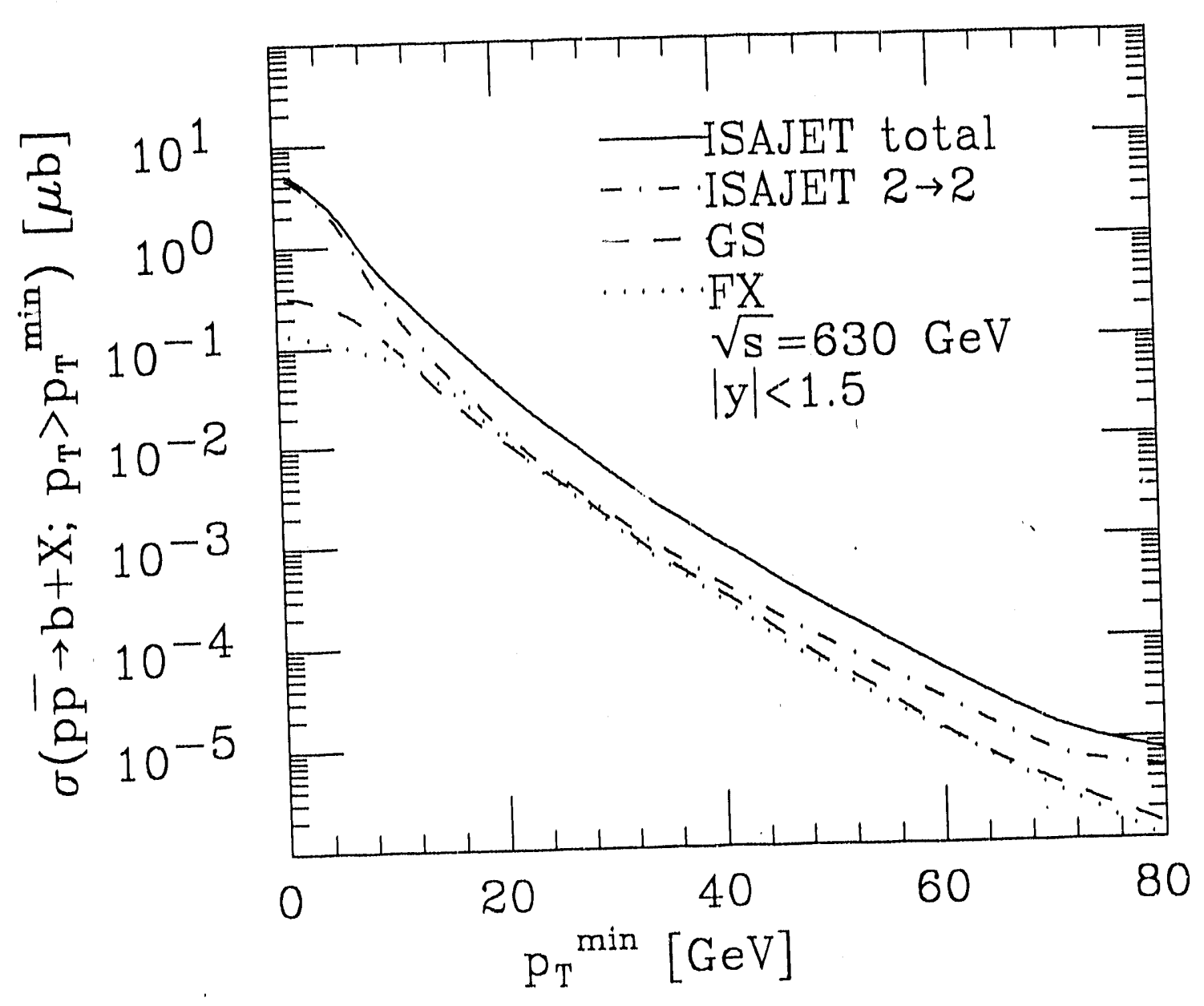

Figure 14 - Cross-sections from "intermediate" ISAJET at $\sqrt{s}=630 \mathrm{GeV}$. 


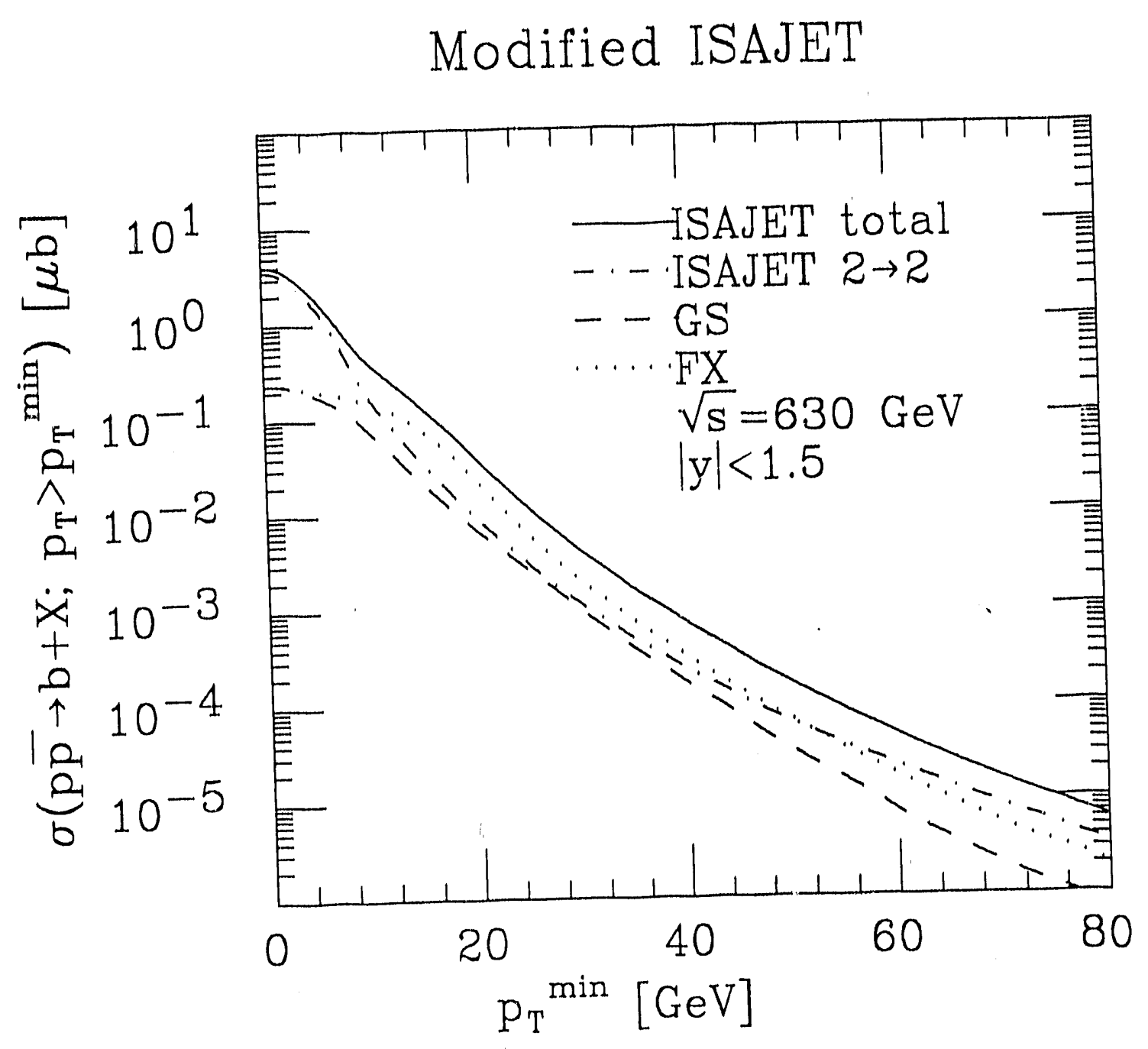

Figure 15 - Cross-sections from "modified" ISAJET at $\sqrt{s}=630 \mathrm{GeV}$. 


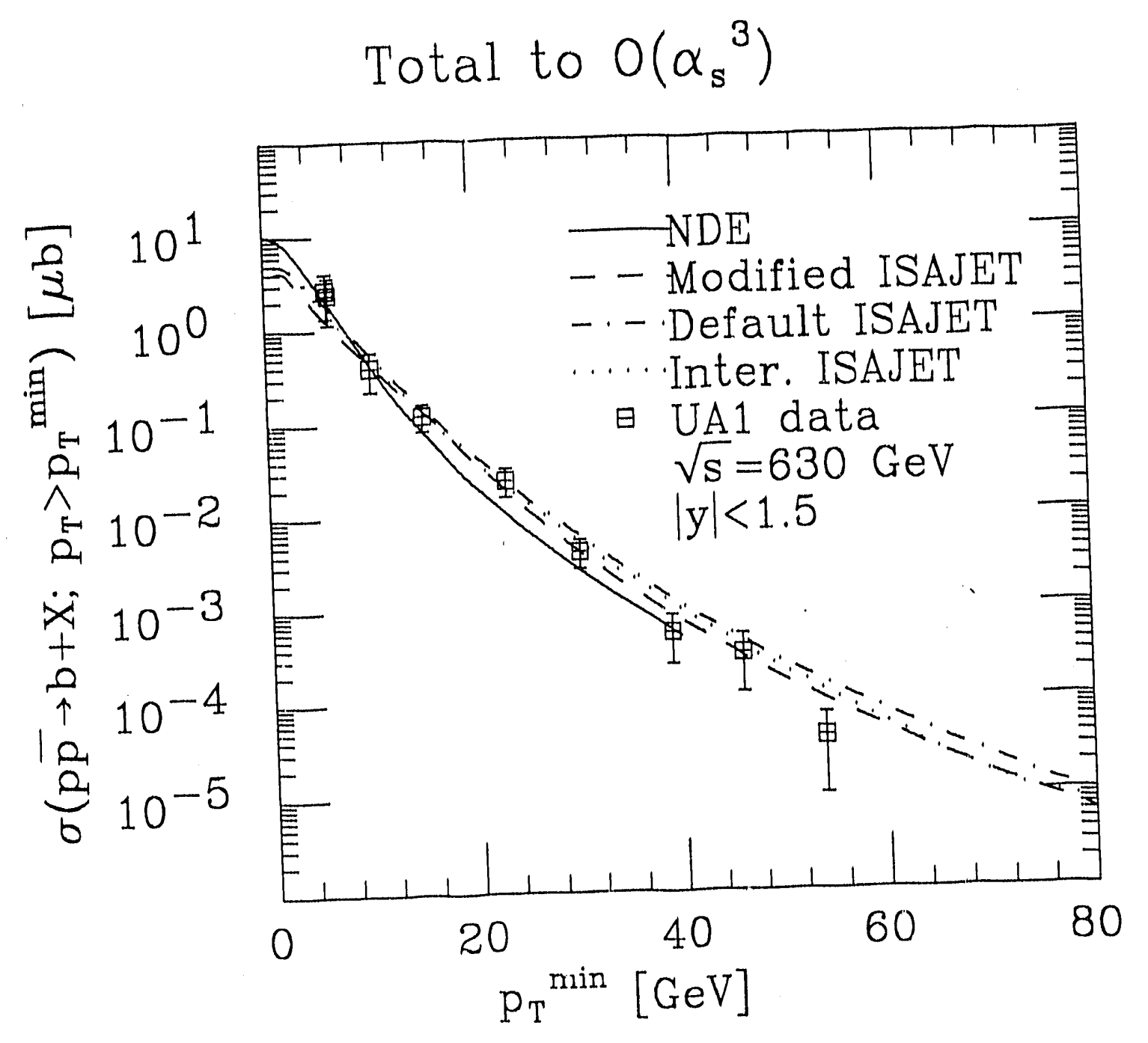

Figure 16 - Total cross-sections for "default", "intermediate", and "modified" ISAJET samples at $\sqrt{s}=630 \mathrm{GeV}$. 


$$
\mathrm{K}=\sigma\left(\mathrm{p}_{\mathrm{T}}>\mathrm{p}_{\mathrm{T}}{ }^{\mathrm{min}}\right)_{\mathrm{O}\left(\alpha_{\mathrm{s}}{ }^{3}\right)} / \sigma_{\mathrm{O}}\left(\alpha_{\mathrm{s}}{ }^{2}\right)
$$

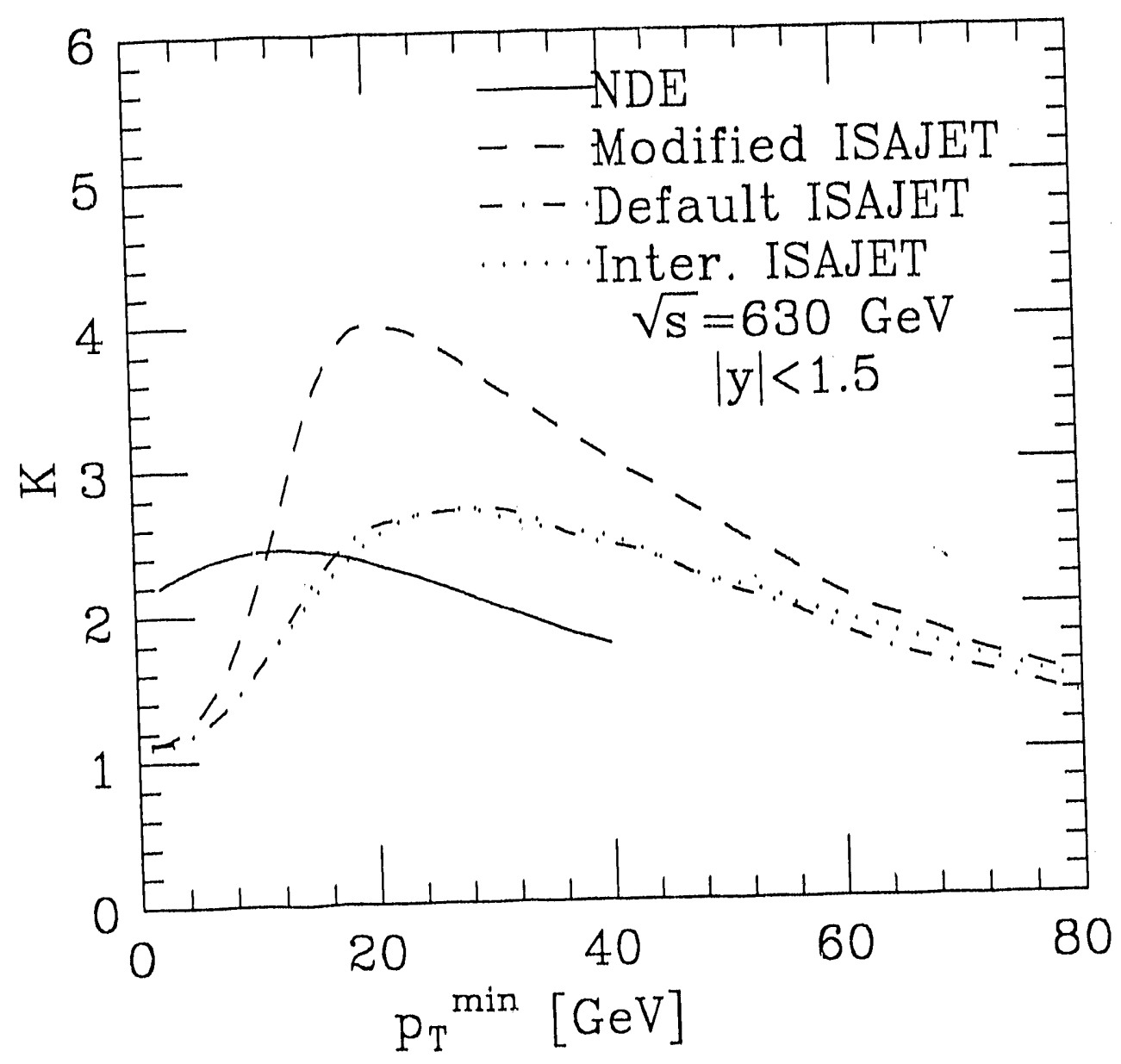

Figure $17-K$ factor at $\sqrt{s}=630 \mathrm{GeV}$ obtained from ISAJET samples. Also presented is the $K$ factor (solid) obtained by dividing the NDE result by the lowest order DFLM cross-sections at the parton level. 


\section{Default ISAJET}

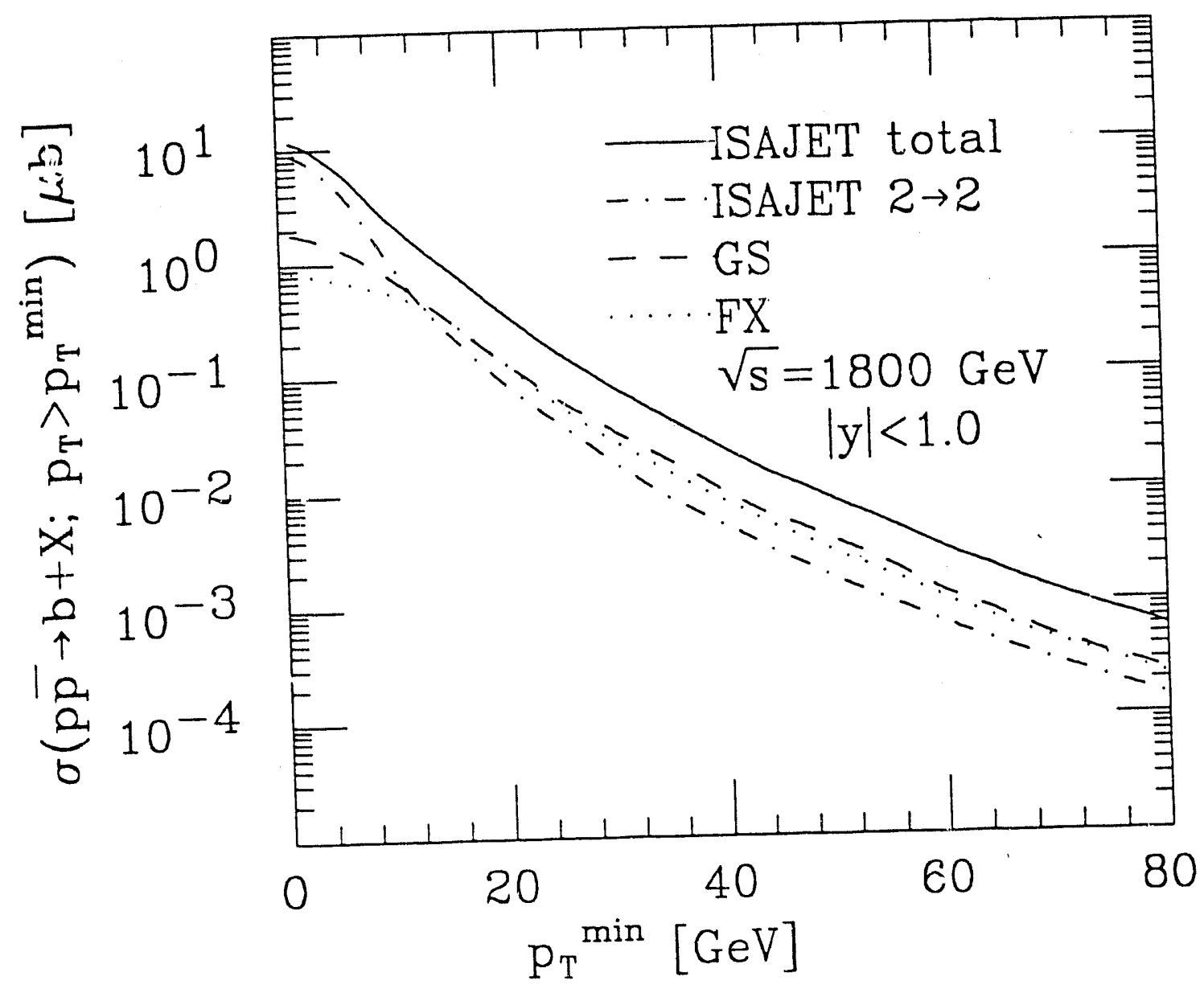

Figure 18 - Cross-sections from "default" ISAJET $\sqrt{s}=1800 \mathrm{GeV} . \sigma(p \bar{p} \rightarrow$ $\left.b+X ; p_{T, b}>p_{T, \text { min }}\right)$ is plotted against $p_{T, \text { min }}$. The rapidity cut on the $b$ quark is $\left|y_{b}\right|<1.0$ at this energy. The solid line :s the sum of the three processes which is the total inclusive cross-section given by ISAJET. 
Intermediate ISAJET

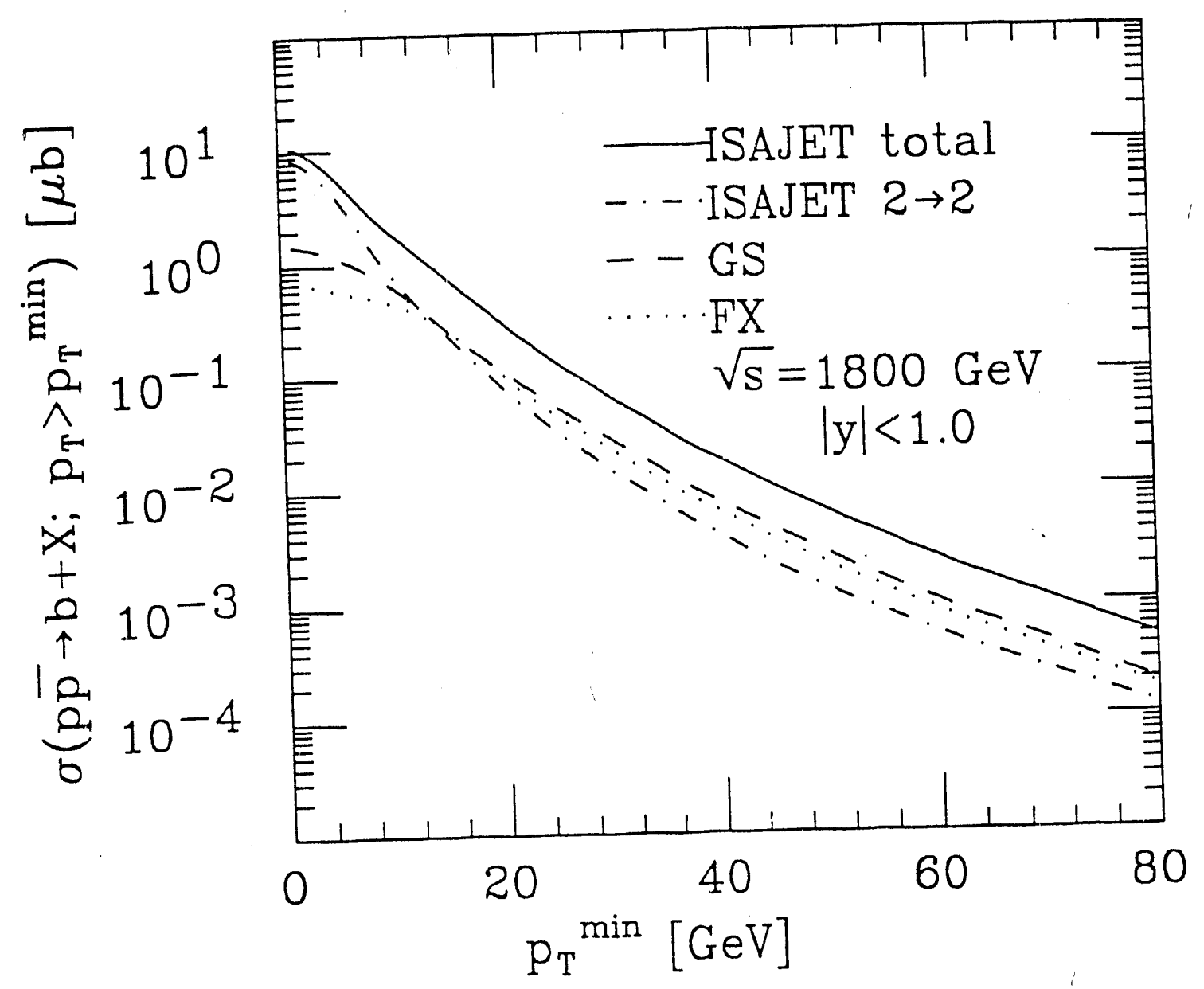

Figure: $19-$ Cross-sections from "intermediate" ISAJET at $\sqrt{s}=1800 \mathrm{GeV}$. 


\section{Modified ISAJET}

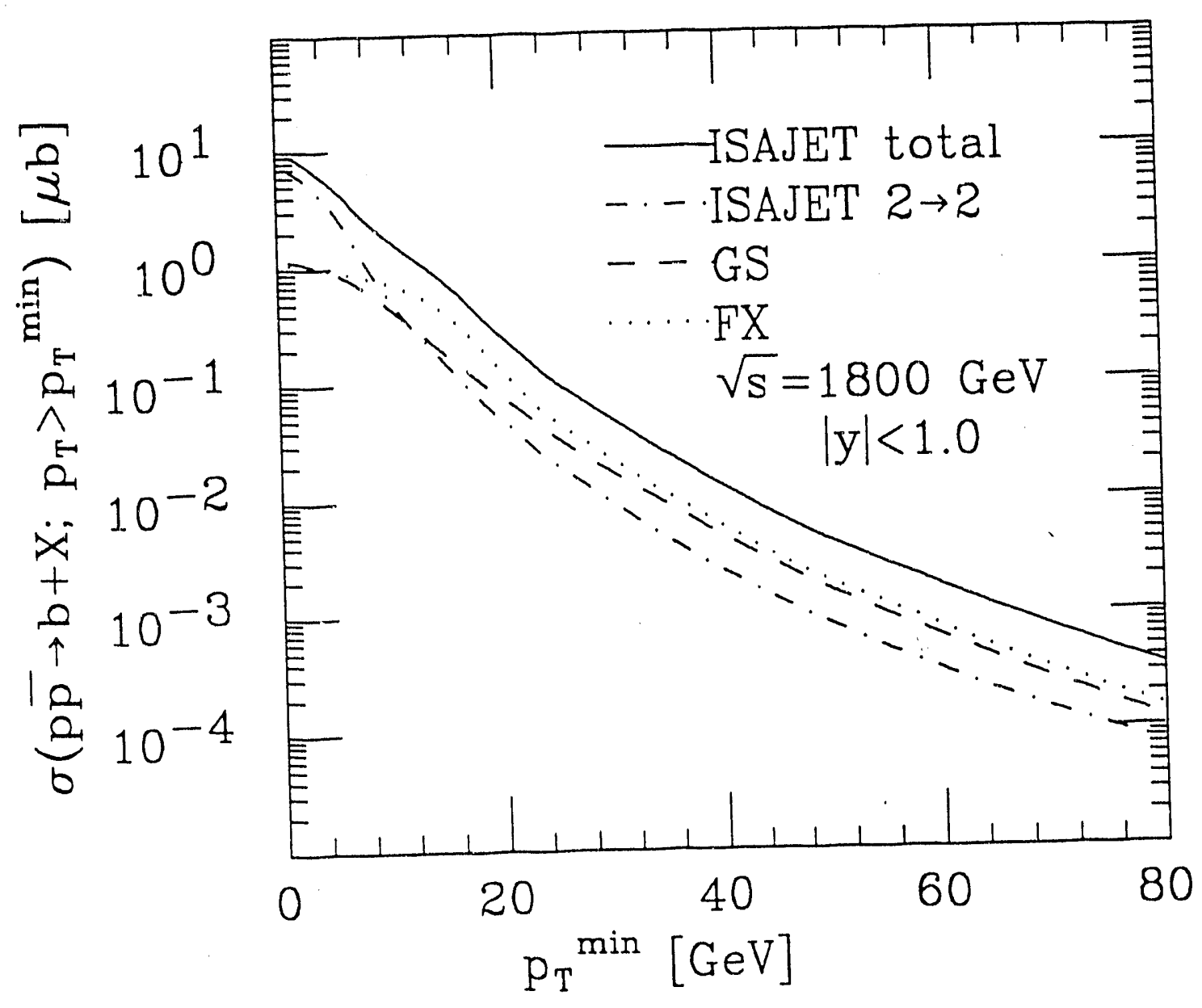

Figure 20 - Cross-sections from "modified" ISAJET at $\sqrt{s}=1800 \mathrm{GeV}$. 


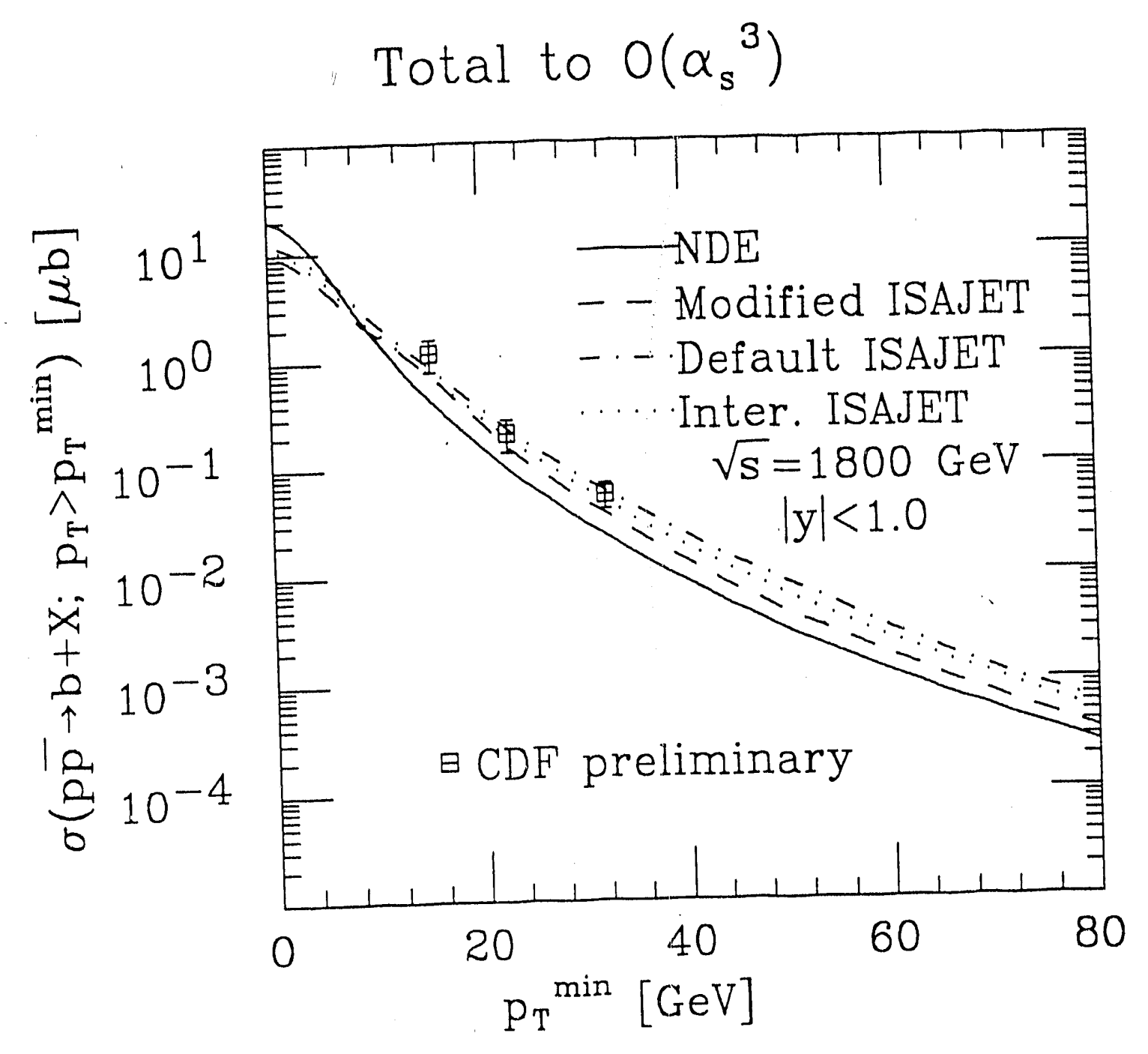

Figure 21 - Total cross-sections for "default", "intermediate", and "modified" ISAJET samples at $\sqrt{s}=1800 \mathrm{GeV}$. 


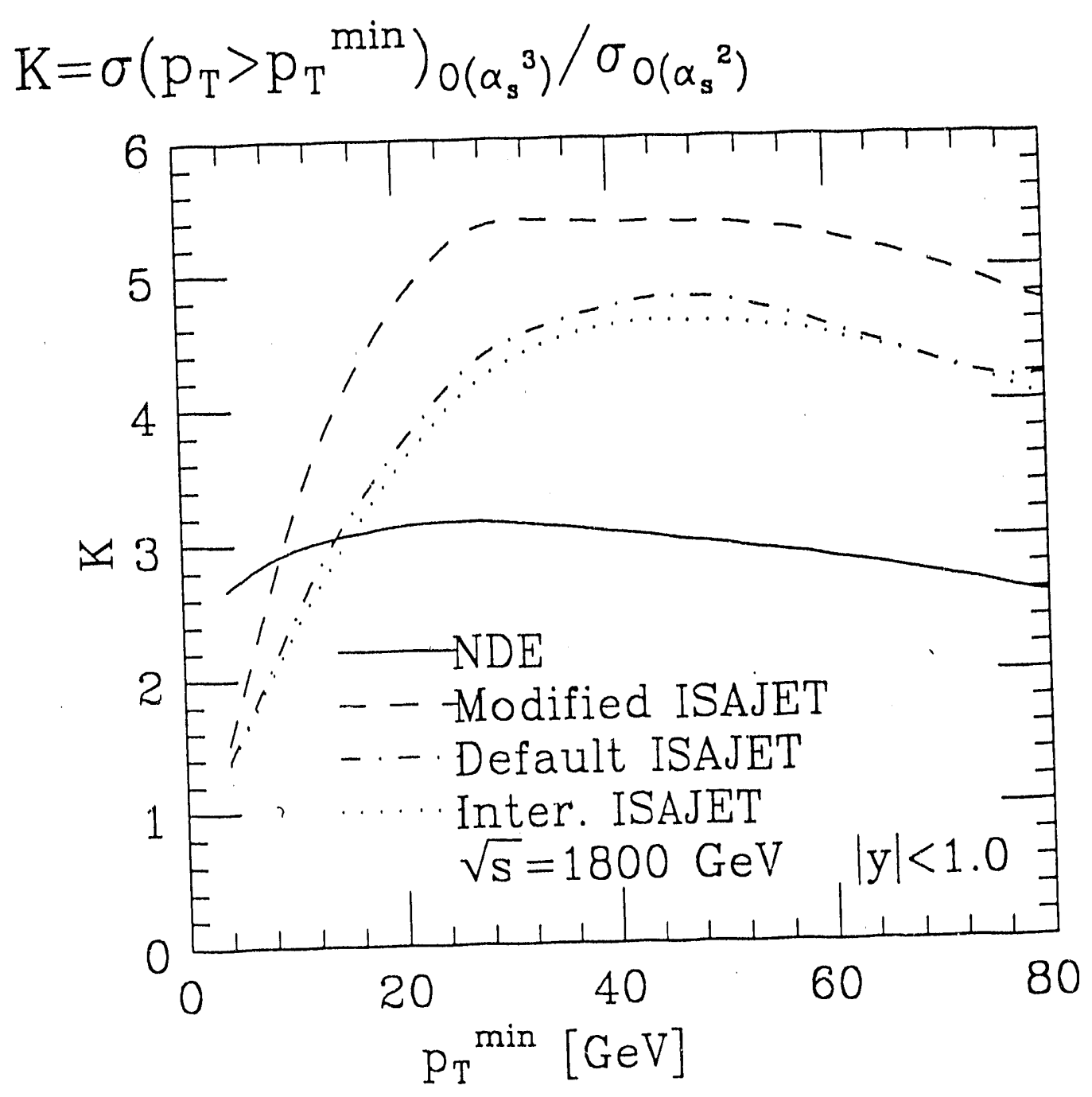

Figure $22-K$ factor at $\sqrt{s}=1800 \mathrm{GeV}$ obtained from ISAJET samples. Also presented is the $K$ factor (solid) obtained by dividing the NDE result by the lowest order DFLM cross-sections at the parton level. 


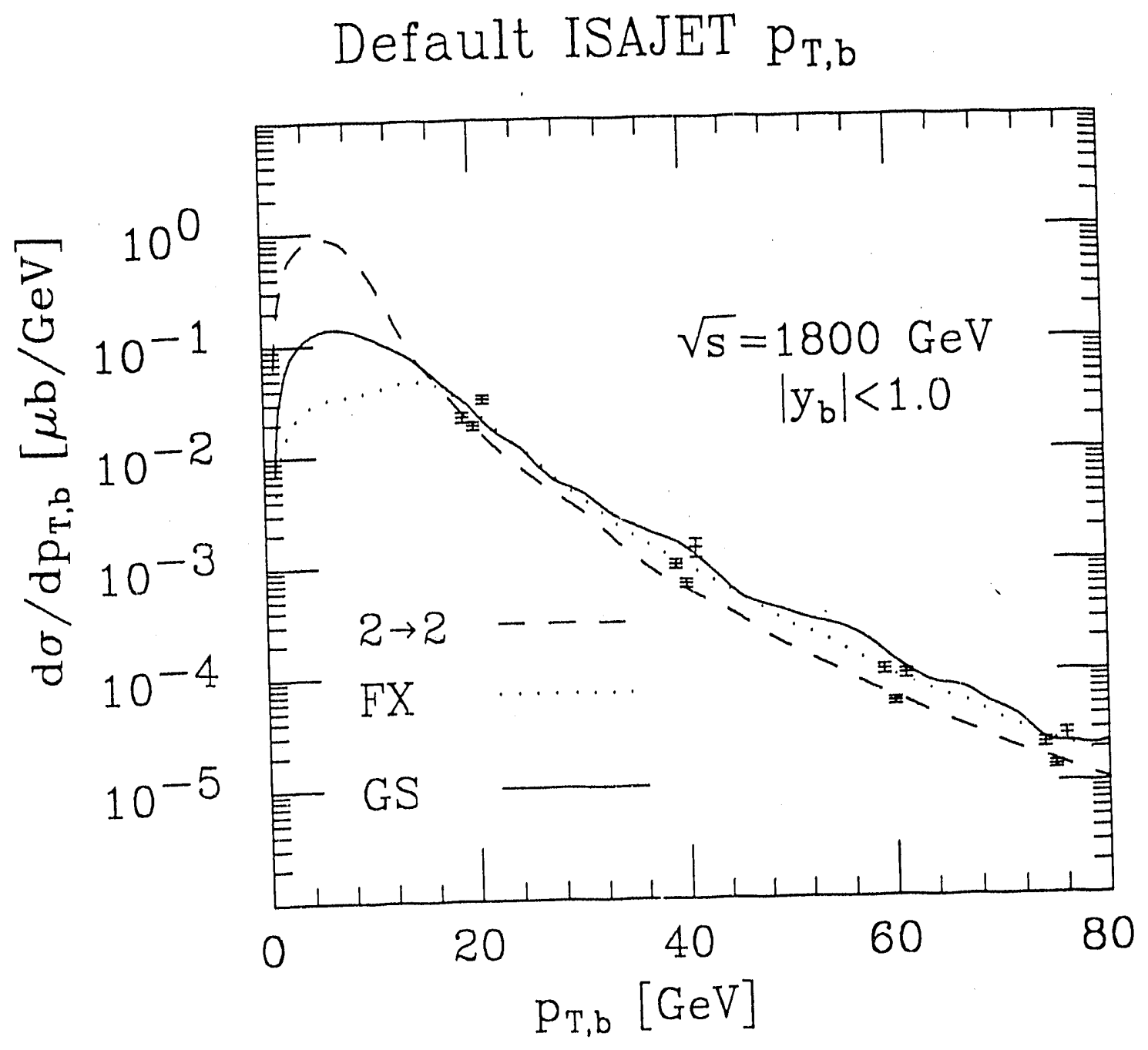

Figure 23 - Transverse momentum distribution from default ISAJET for the $b$ quark with $\left|y_{b}\right|<1$. Sample points are shown at $p_{T, b}$ values of $20,40,60$, and $75 \mathrm{GeV}$. The flavor excitation points are shifted slightly to the left and the gluon splitting values are shifted to the right. 


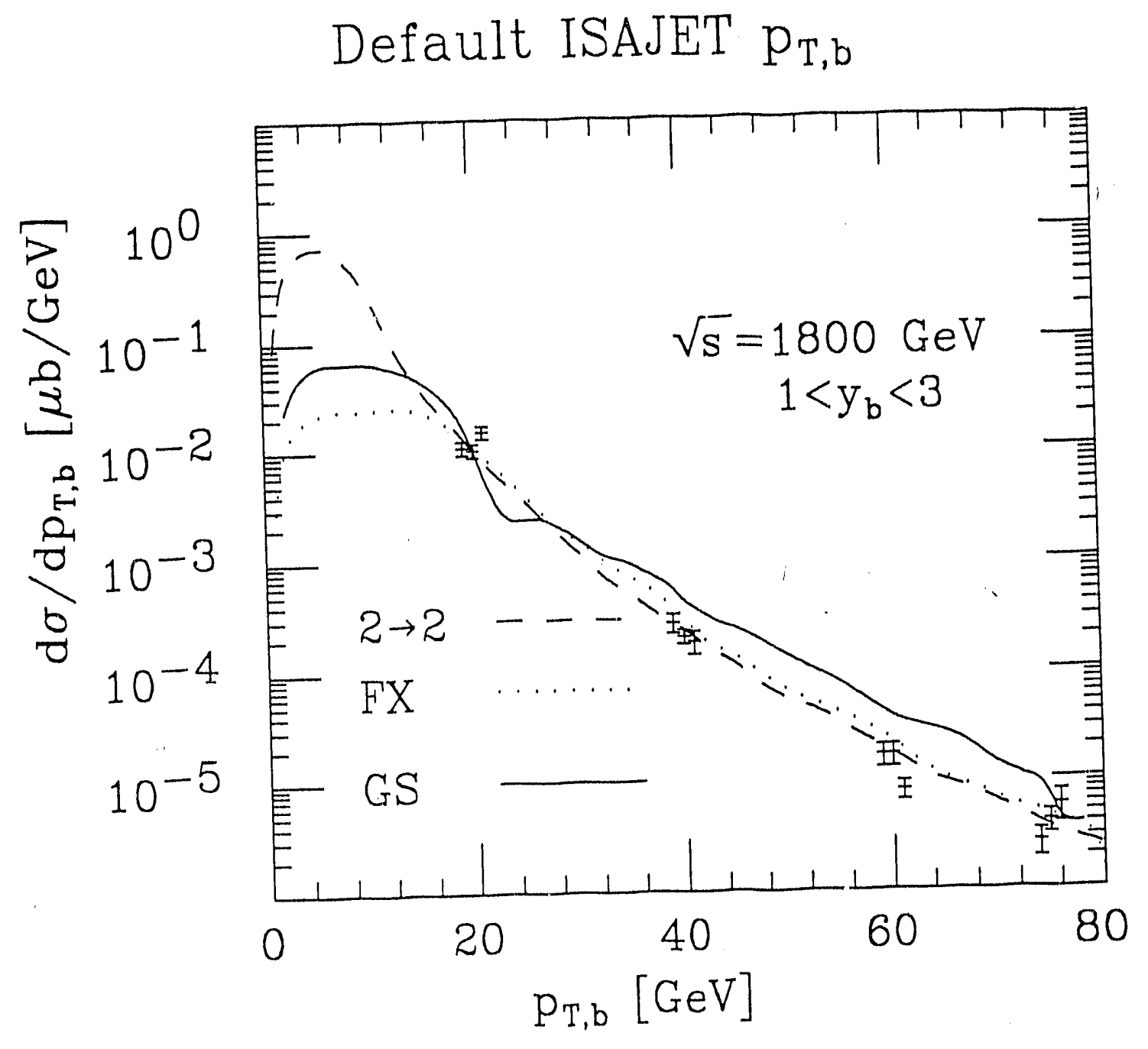

Figure 24 - Transverse momentum distribution from default ISAJET for the $b$ quark with $1<y_{b}<3$. Sample points are shown at $p_{T, b}$ values of $20,40,60$, and $75 \mathrm{GeV}$. The flavor excitation points are shifted slightly to the left and the gluon splitting values are shifted to the right. 


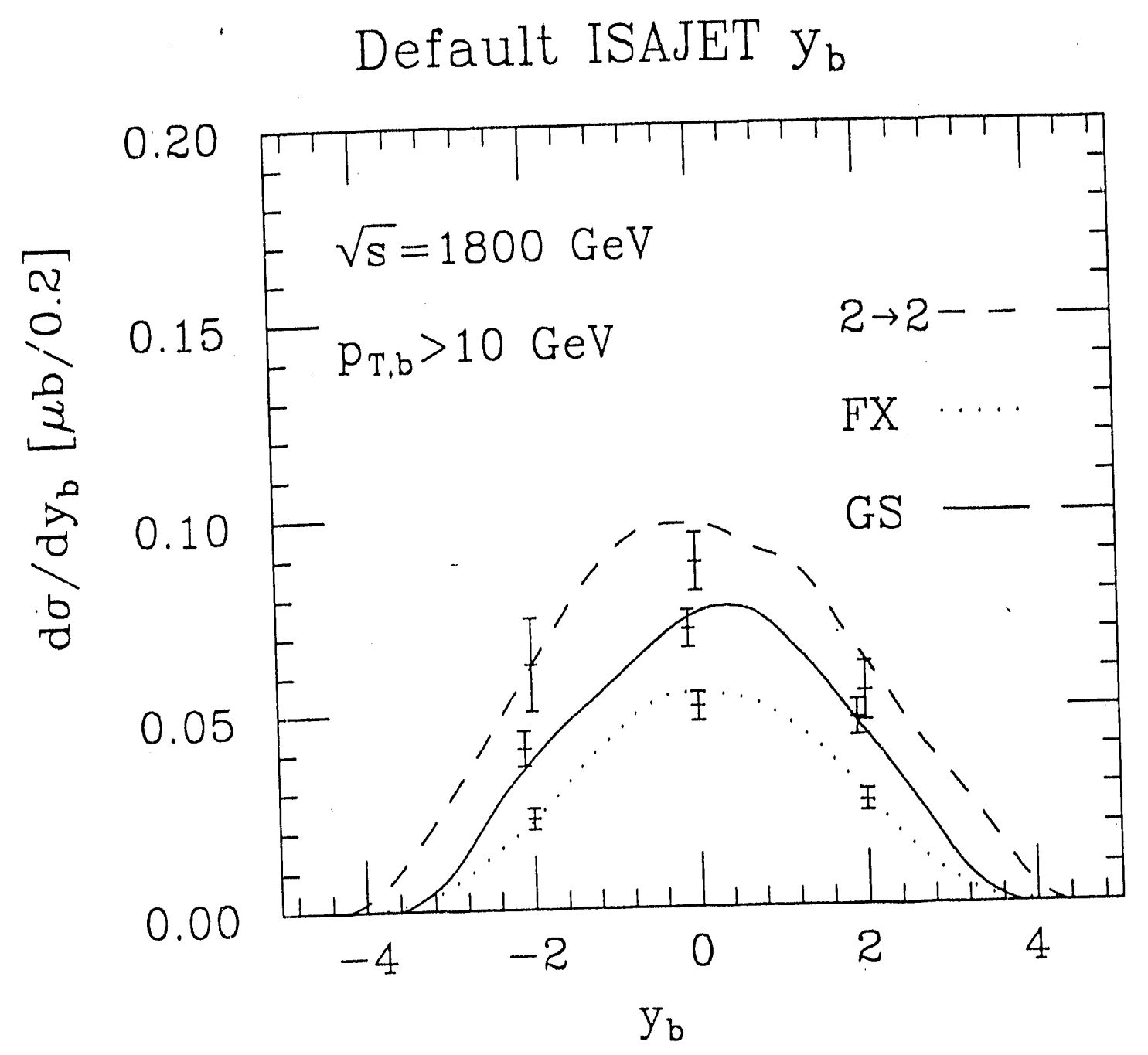

Figure 25 - Rapidity distribution from default ISAJET for the $b$ quark with $p_{T, b}>10 \mathrm{GeV}$. Sample points are shown at rapidity values of $-2,0$, and 2 with the gluon splitting point shifted slightly to the left. 


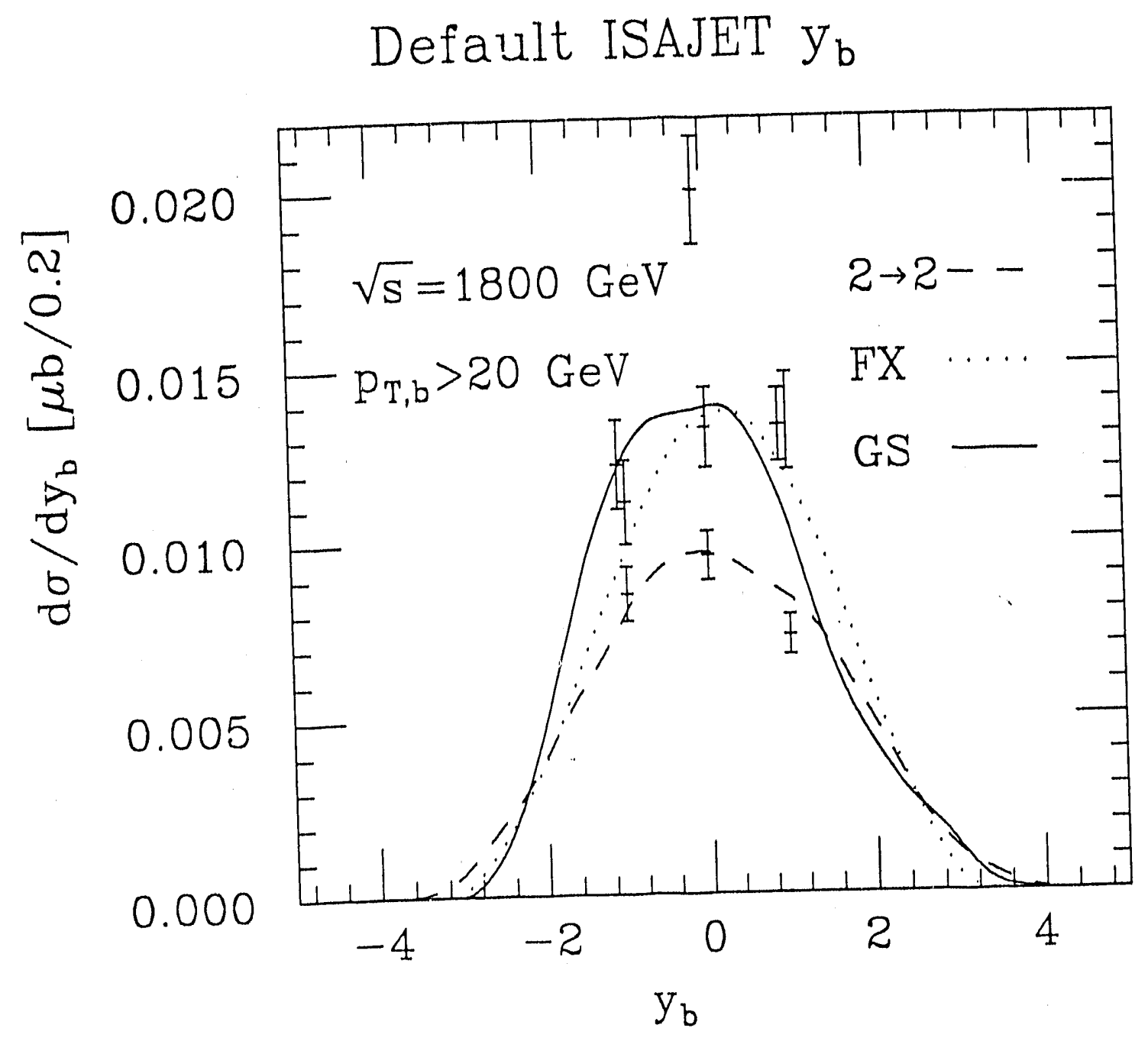

Figure 26 - Rapidity distribution from default ISAJET for the $b$ quark with $p_{T, b}>20 \mathrm{GeV}$. Sample points are shown at rapidity values of $-1,0$, and 1 with the gluon splitting point shifted slightly to the left. 


\section{Default ISAJET $\mathrm{p}_{\mathrm{T}}{ }^{\text {pair }}$}

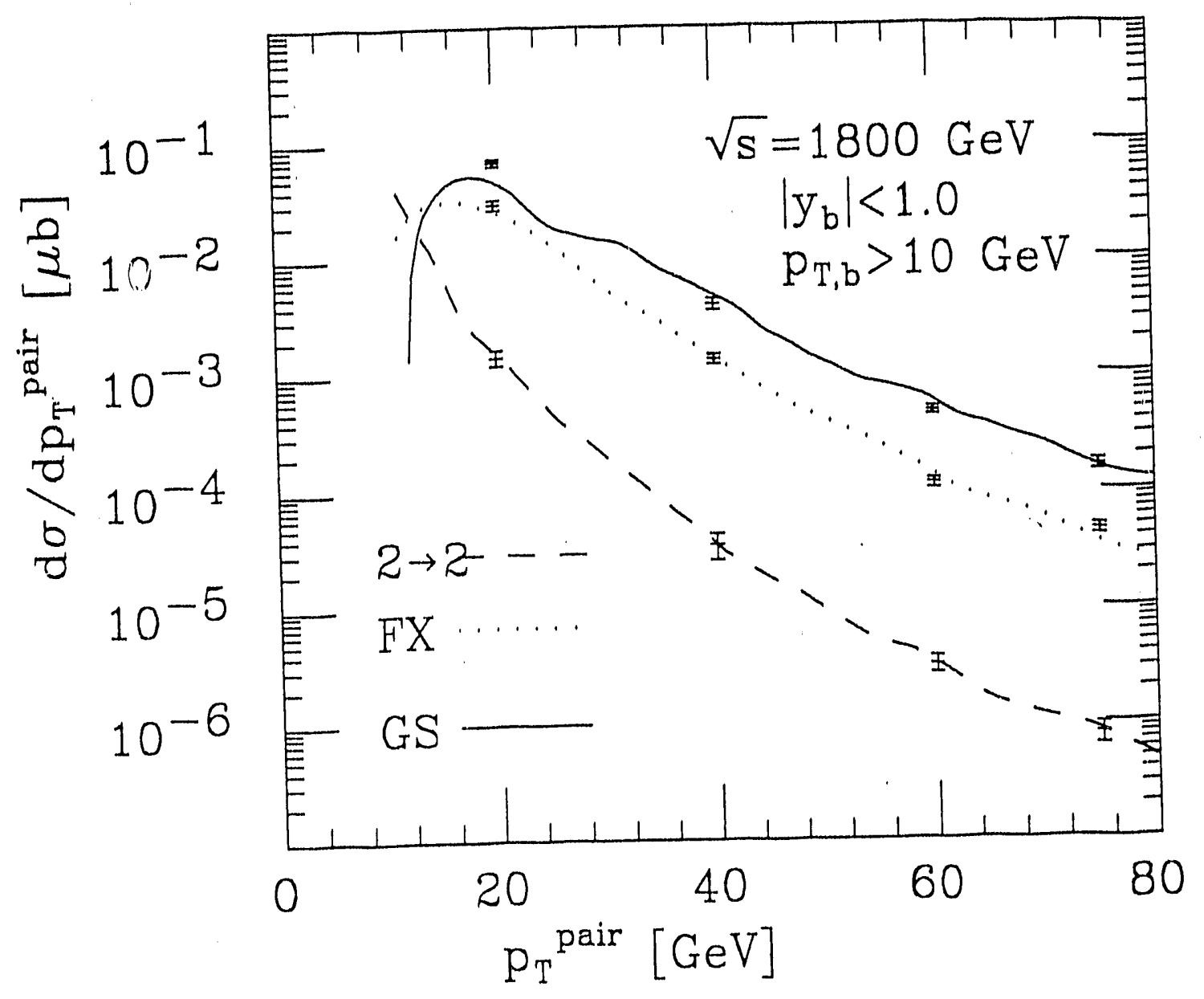

Figure 27 - Transverse momenturn distribution from default ISAJET for $p_{T}^{\text {pair }}$, the vector sum of the transverse momenta of the $b$ and the $\bar{b} . \quad\left(\left|y_{b}\right|<1\right.$.) Sample points are shown at $p_{T}^{\text {pair }}$ values of $20,40,60$, and $75 \mathrm{GeV}$. 


\section{Default ISAJET $\mathrm{p}_{\mathrm{T}}^{\text {pair }}$}

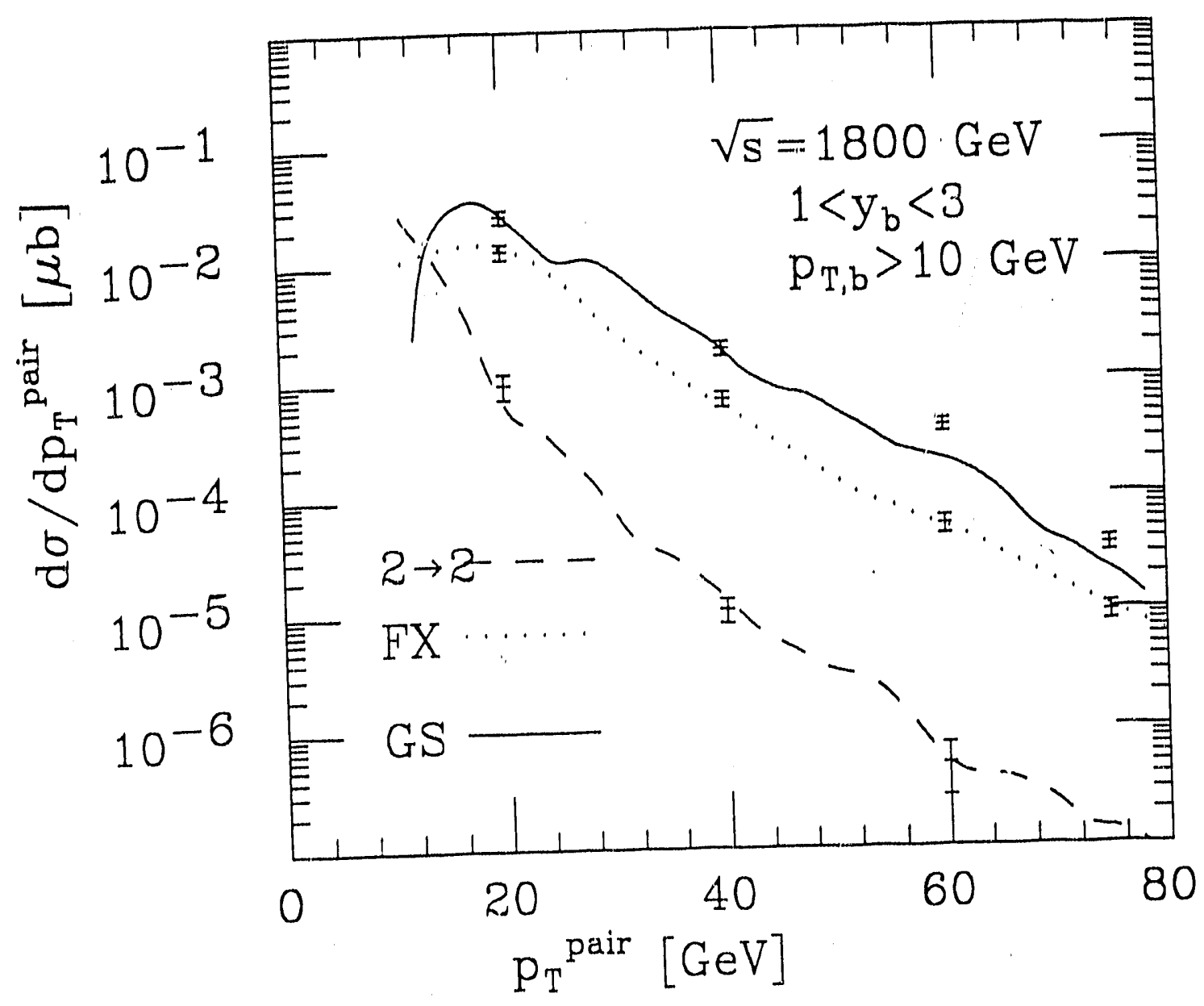

Figure 28 - Transverse momentum distribution irom default ISAJET for $p_{T}^{\text {pair }}$, the vector sum of the transverse momenta of the $b$ and $\bar{b}$. $\left(1<y_{b}<3\right.$.) Sample points are shown at $p_{T}^{\text {pair }}$ values of $20,40,60$, and $75 \mathrm{GeV}$. 
Default ISAJET $\Delta \mathrm{y}$

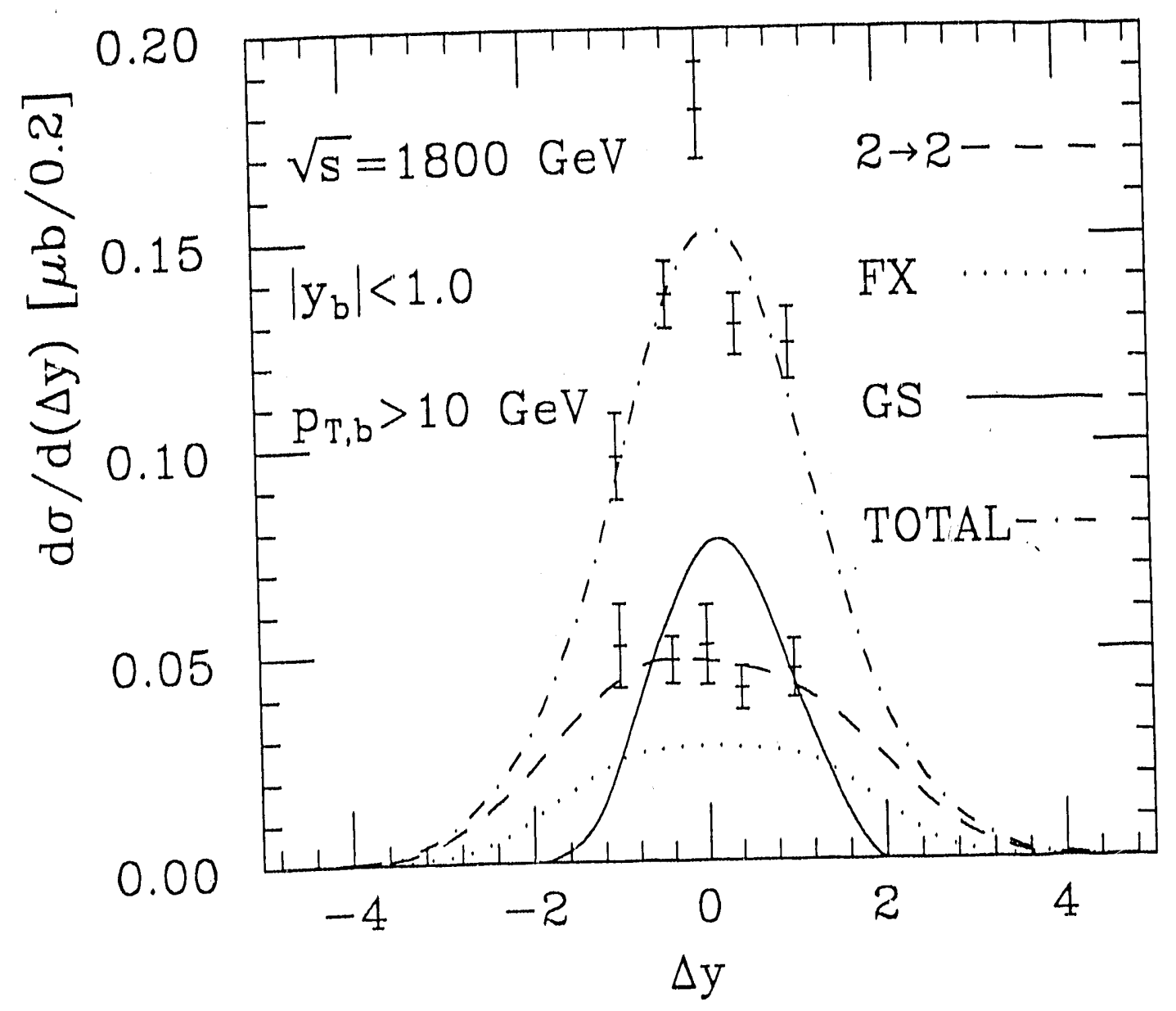

Figure 29 - Rapidity difference distribution of the heavy flavor pair for each production mode. The $b$ quark has $\left|y_{b}\right|<1$ and transverse momentum larger than $10 \mathrm{GeV}$. Sample points are shown at rapidity values of $-1,-0.4,0,0.4$, and 1 for the lowest order and total cross-sections. 


\section{Default ISAJET $\Delta y$}

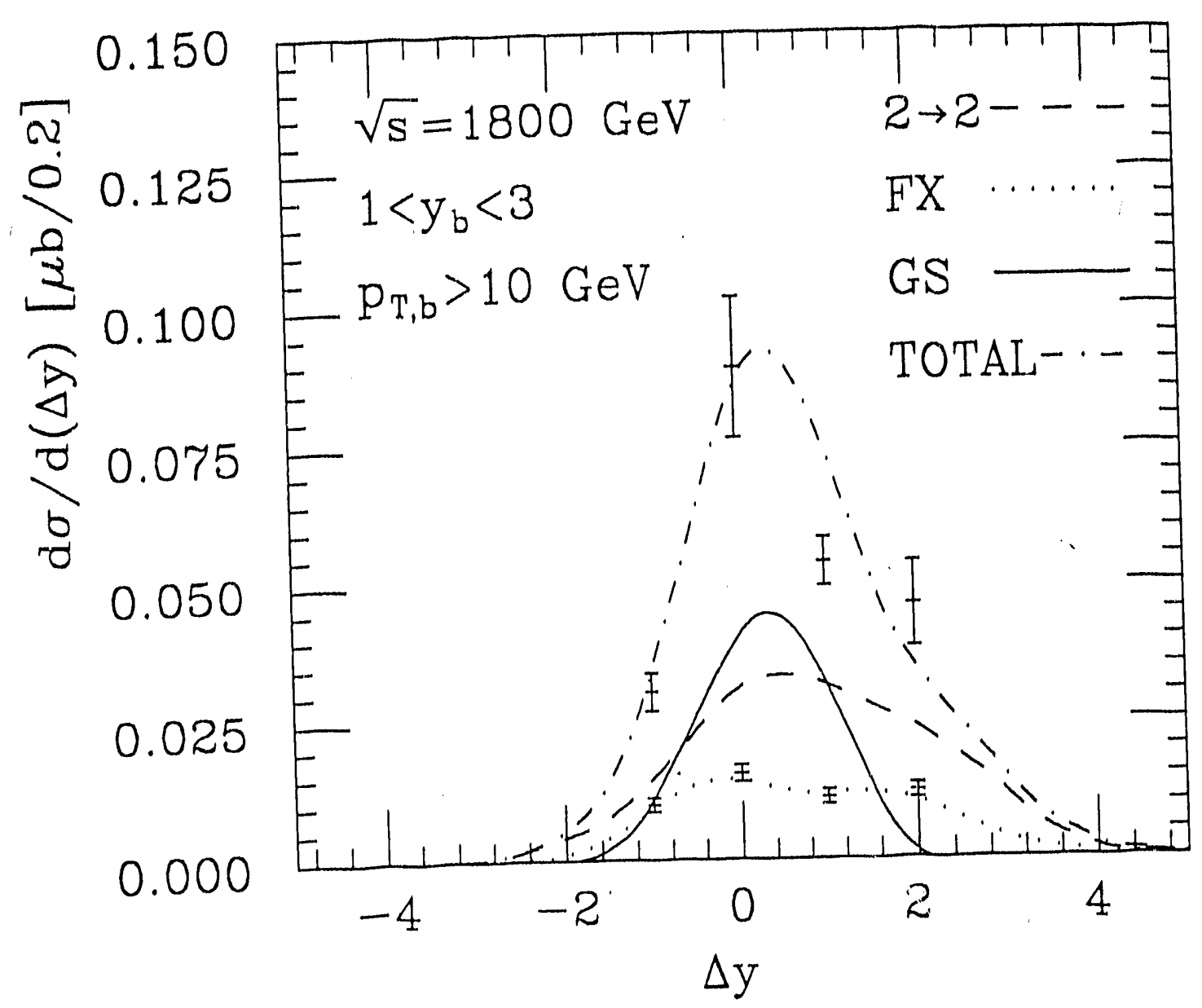

Figure 30 - Rapidity difference distribution of the heavy flavor pair for each production mode. The $b$ quark has rapidity between 1 and 3 and transverse momentum larger than $10 \mathrm{GeV}$. Sample points are shown at rapidity values of $-1,0,1$, and 2 for the flavor excitation and total cross-sections. 


\section{Default ISAJET $\Delta y$}

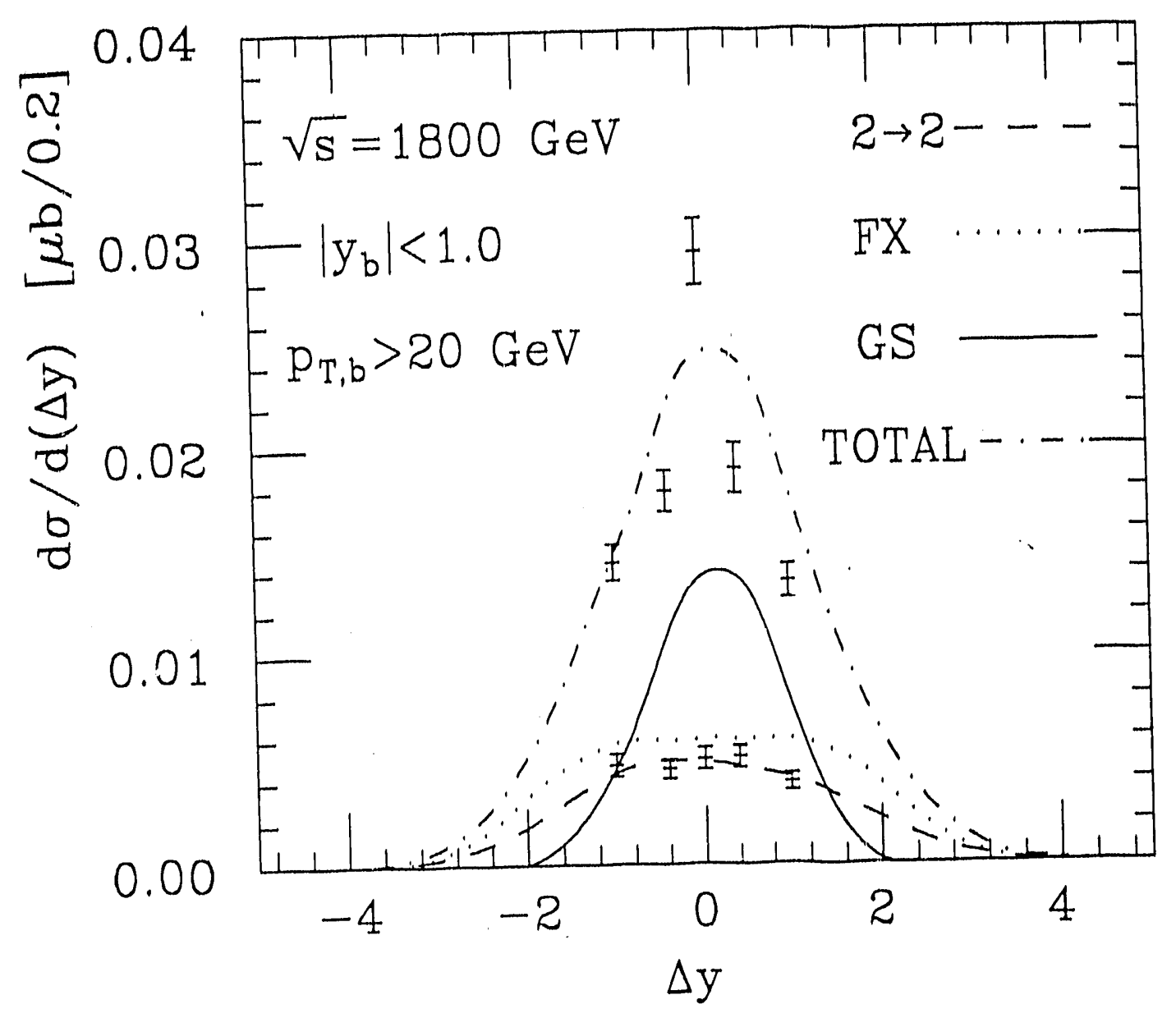

Figure 31 - Rapidity difference distribution of the heavy flavor pair for each production mode. The $b$ quark has $\left|y_{b}\right|<1$ and transverse momentum larger than 20 $\mathrm{GeV}$. Sample points are shown at rapidity values of $-1,-0.4,0.4$, and 1 for the lowest order and total cross-sections. 


\section{Default ISAJET $\Delta y$}

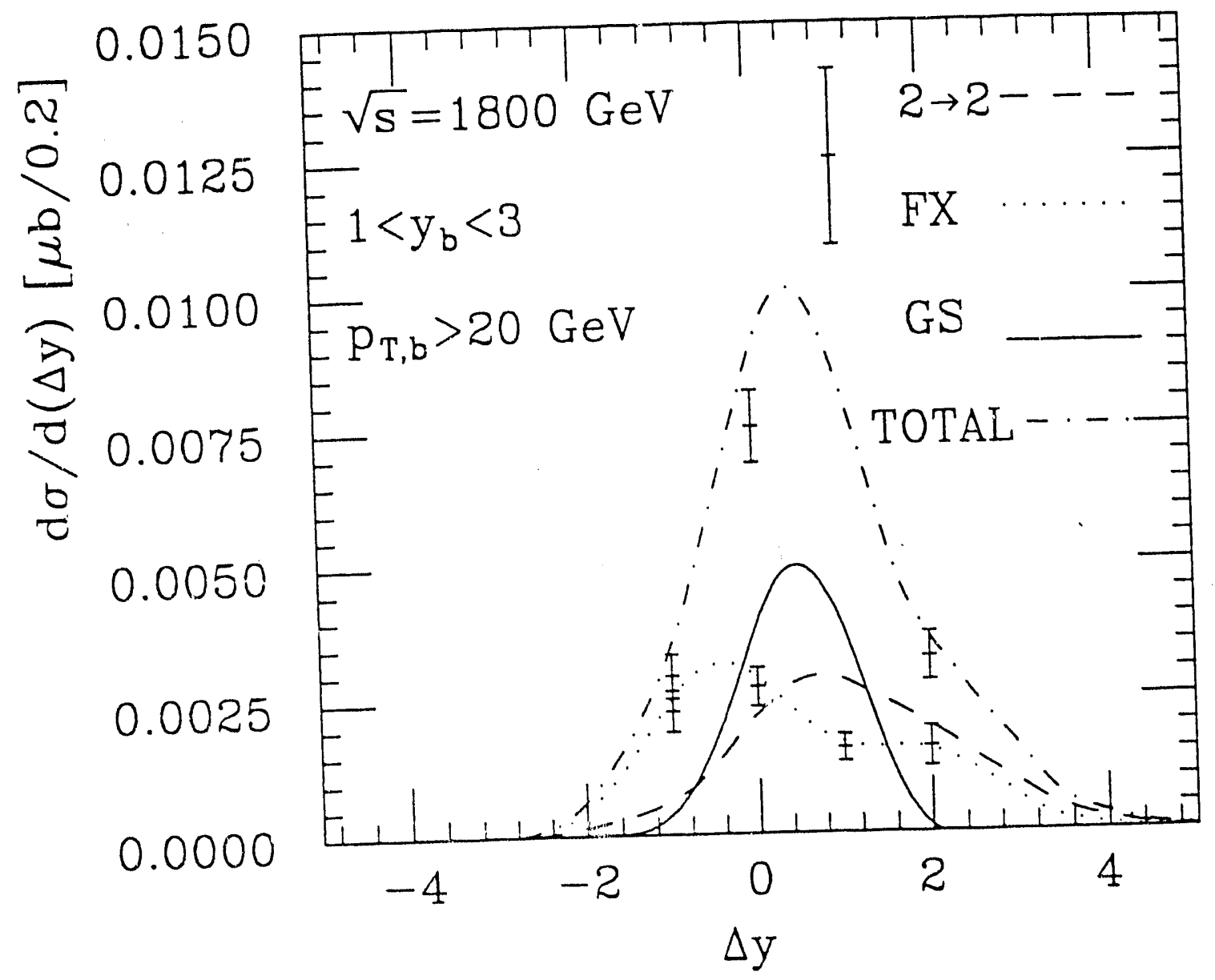

Figure $3:-$ Rapidity difference distribution of the heavy flaw pair for each production mode. The $b$ quark has rapidity between 1 and 3 and transverse momentum larger than $20 \mathrm{GeV}$. Sample points are shown at rapidity values of $-2,-1,1$, and 2 for the flavor excitation and total cross-sections. 
Default ISAJET $\Delta \phi$

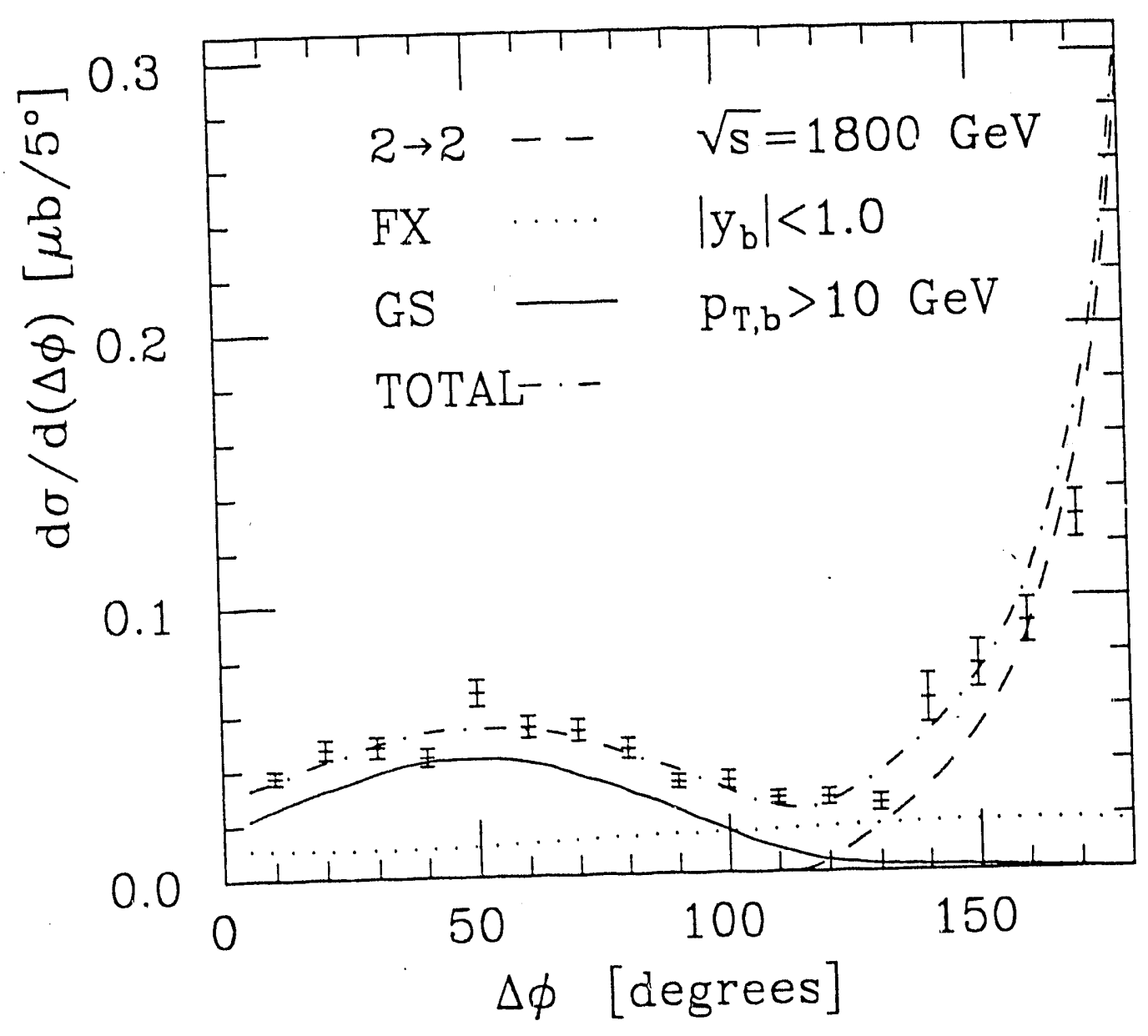

Figure 33 - Azimuthal angle distribution of $t$ l e heavy flavor pair for each product:on mode. The $b$ quark has $\left|y_{b}\right|<1$ and transverse momentum larger than $10 \mathrm{GeV}$. Actual values with the ISAJET calculated errors are shown for the total cross-section. 


\section{Default ISAJET $\Delta \phi$}

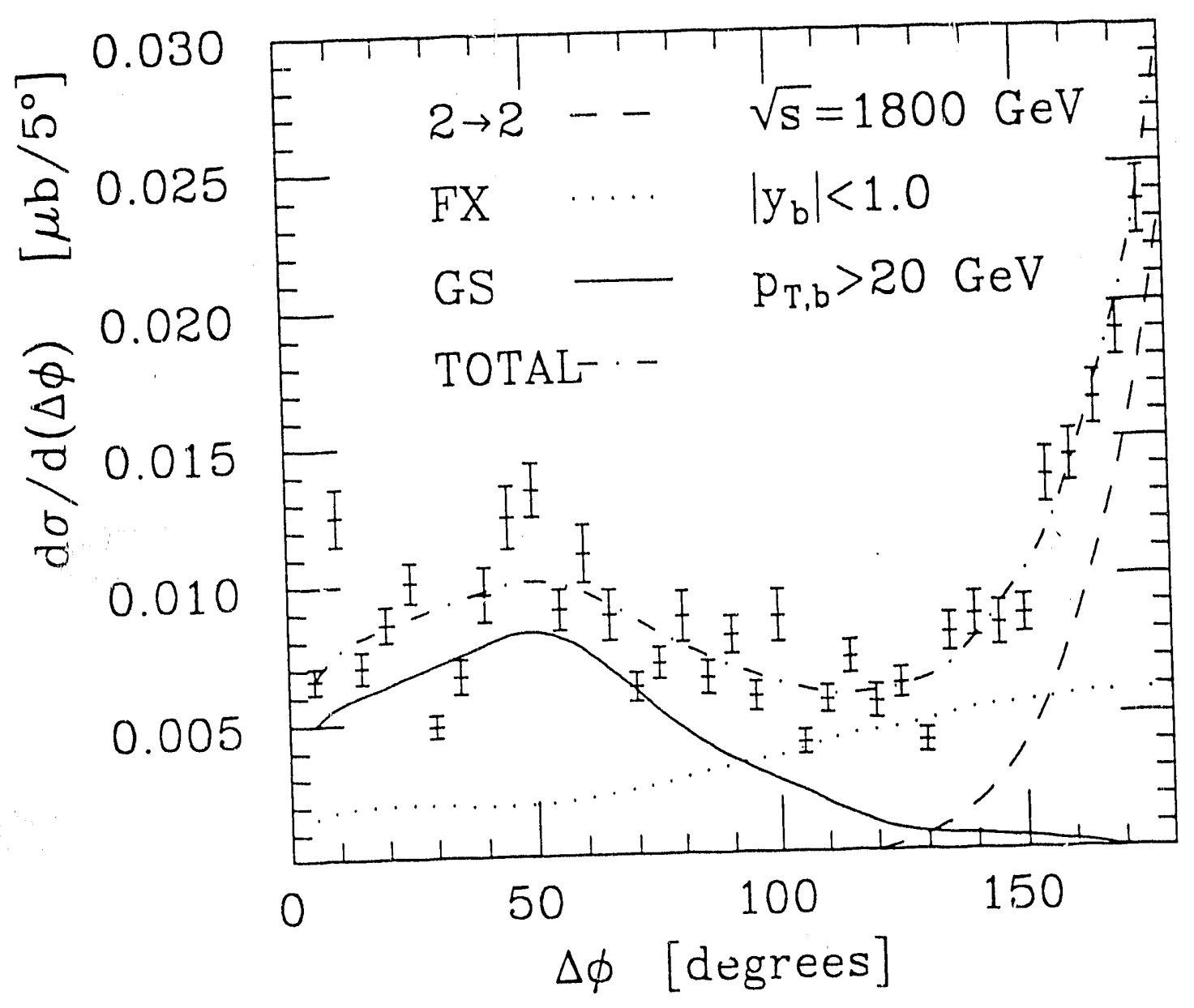

Figure 34 - Azimuthal angle distribution of the heavy flavor pair for each production mode. The $b$ quark has $\left|y_{b}\right|<1$ and transverse momentum larger than $20 \mathrm{GeV}$. Actual values with the ISAJET calculated errors are shown for the total cross-section. 


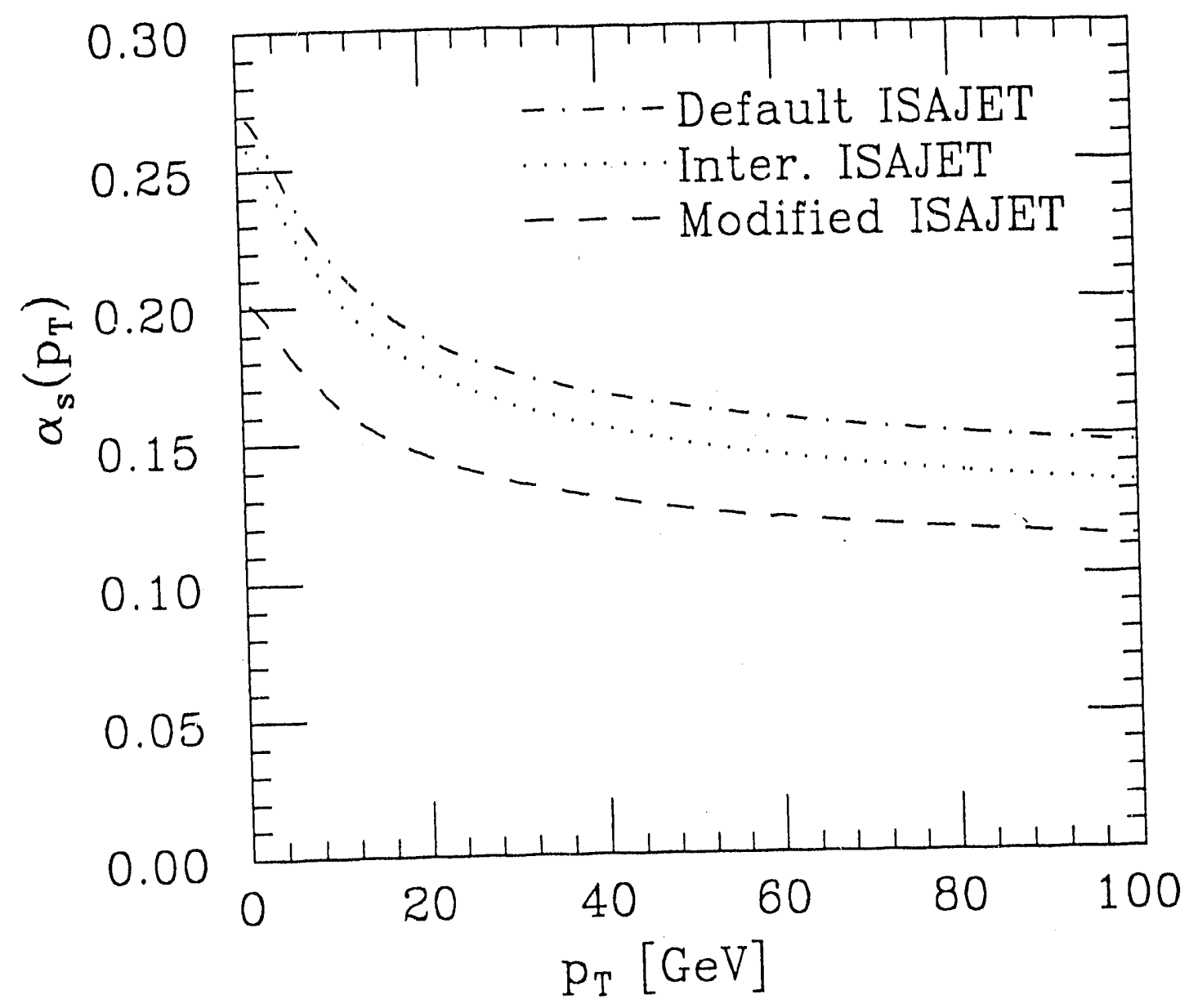

Figure 35 - The strong coupling strength $\alpha_{s}$ is shown as a function of $p_{T}$ for each of the three samples: "default" ISAJET (dot-dash), "intermediate" ISAJET (dots), and "modified" DFLM ISAJET (dashes). For these curves the masses and scale choice are fixed in order to study the effect of the different functional forms for the coupling constant. $m_{b}=4.75 \mathrm{GeV}, m_{\ell}=60 \mathrm{GeV}, \Lambda_{4}=260 \mathrm{MeV}$, and $Q^{2}=m_{b}^{2}+p_{T}^{2}$. 


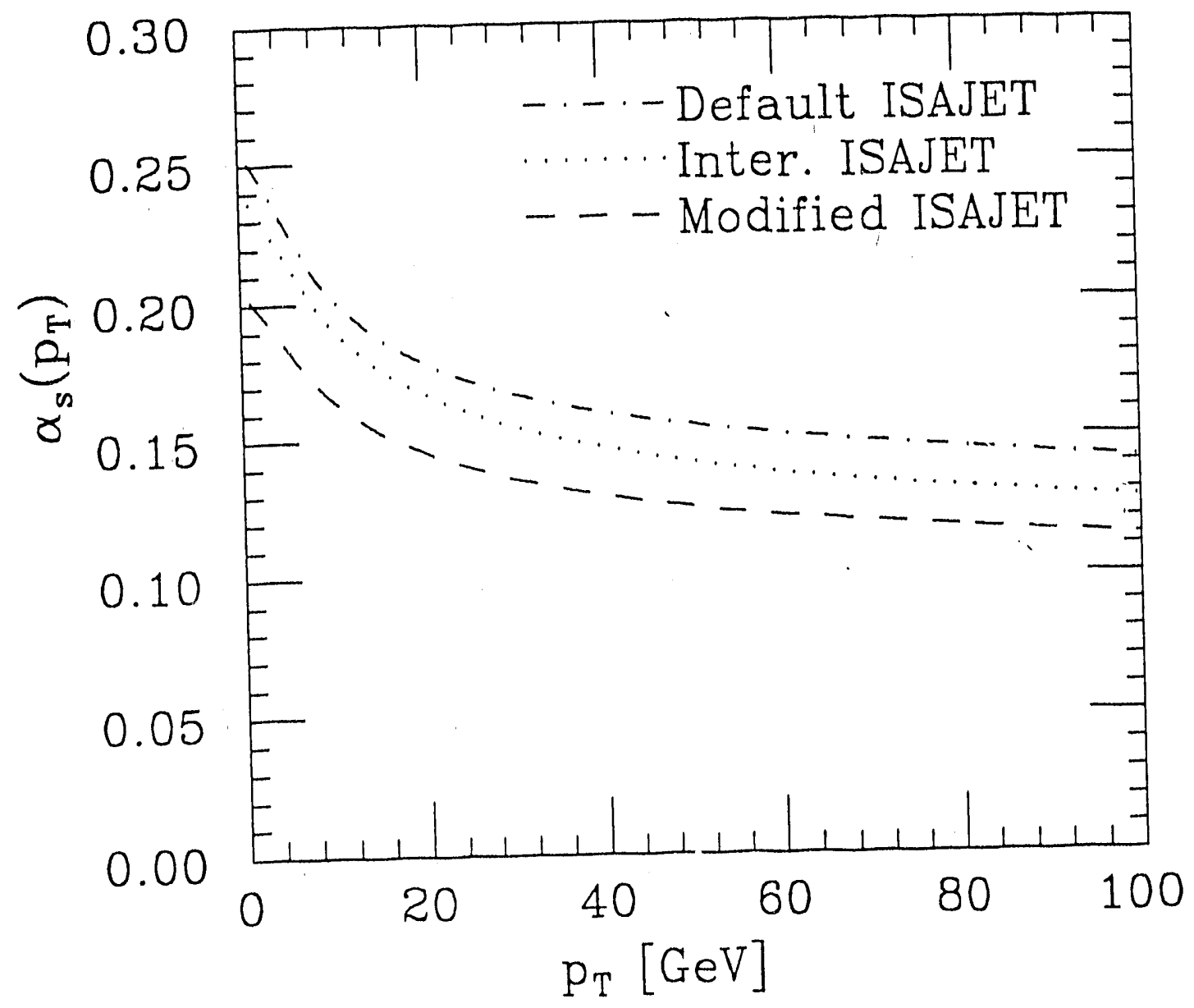

Figure 36 - The strong coupling strength $\alpha$, vs. $p_{T}$ is shown using the appropriate parameters for each of the three data samples as described in the beginning of section 3 . 


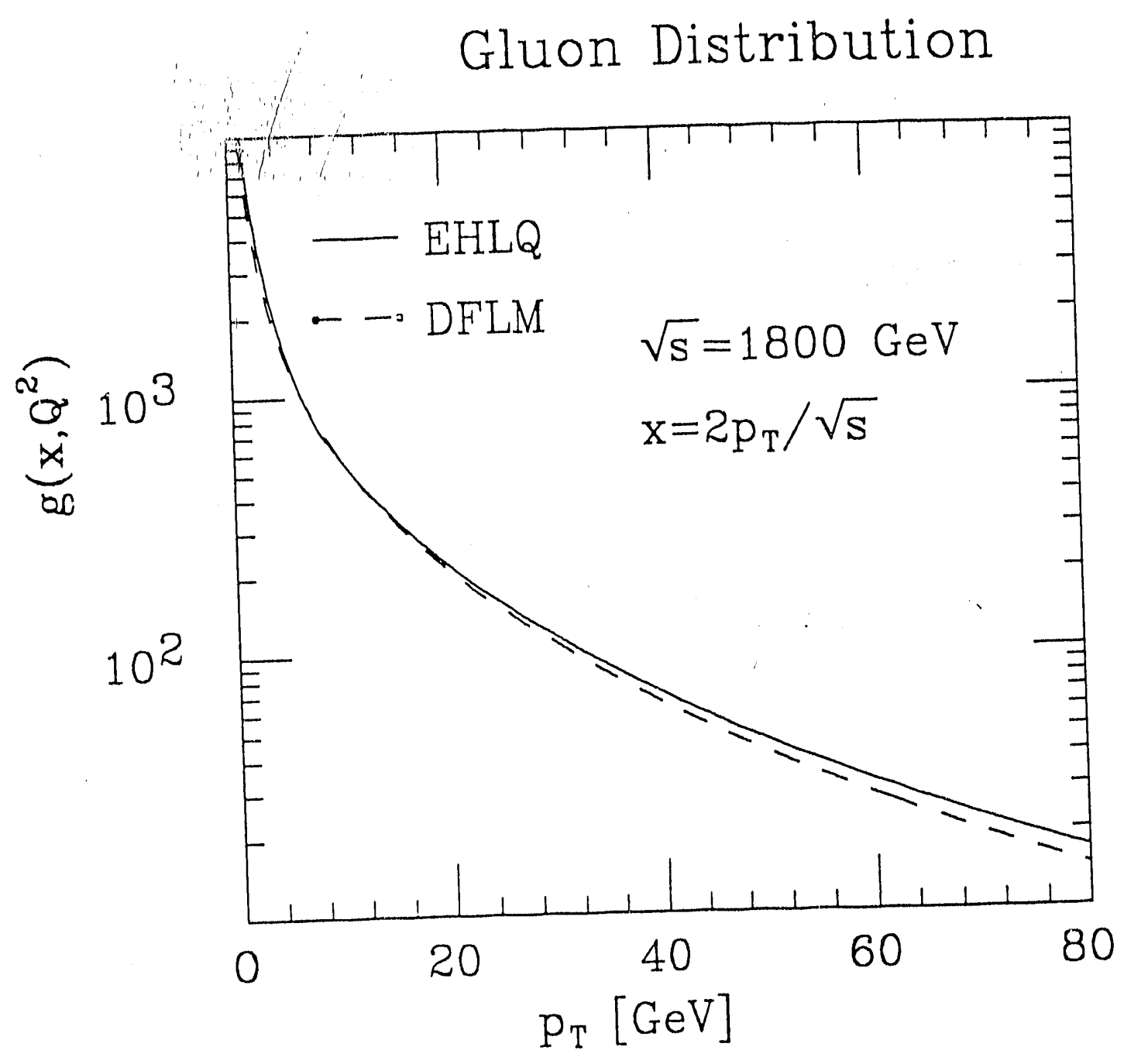

Figure 37 - EHLQ (solid line) and DFLM (dashec' line) gluon distribution as a function of $p_{T}$. The function $g\left(x, Q^{2}\right)$ is evaluated by taking $x=2 p_{T} / \sqrt{s}$. $\varepsilon^{3}=p_{T}^{2}+m_{b}^{2}$ for the DFLM curve $\left(m_{b}=4.75 \mathrm{GeV}\right)$ and the default ISAJET scale is evaluated at $\Delta y=0$ for the 2 to 2 hard scatter which yields $Q^{2}=\max \left(4 m_{b}^{2}, 4 p_{T}^{2} / 3\right)$ where $m_{b}=4.9 \mathrm{GeV}$. 


\section{Bottom distribution}

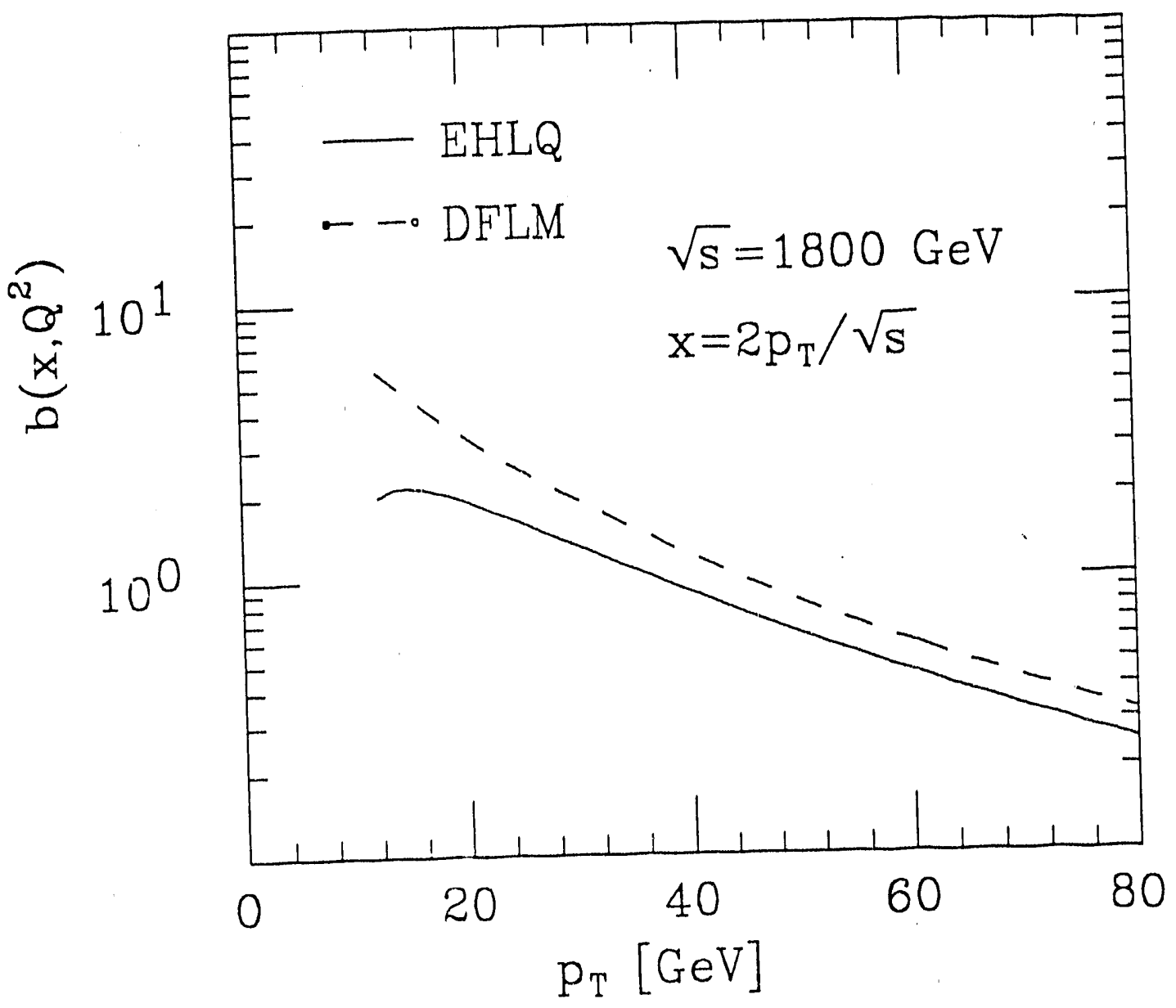

Figure 38 - EHLQ (solid line) and DFLM (dashed line) bottom distribution as a function of $p_{T}$. The function $g\left(x, Q^{2}\right)$ is evaluated by taking $x=2 p_{T} / \sqrt{s}$. $Q^{2}=p_{T}^{2}+m_{b}^{3}$ for the DFLM curve $\left(n_{b}=4.75 \mathrm{GeV}\right)$ and the default ISAJET scale is evaluated at $\Delta y=0$ for the 2 to 2 hard scatter which yields $Q^{2}=\max \left(4 m_{b}^{2}, 4 p_{T}^{2} / 3\right)$ where $m_{b}=4.9 \mathrm{GeV}$. 


\section{REFERENCES}

1. T. Matsuura, S.C. van der Marck, and W.L. van Neerven, Phys. Lett. 211B, 171 (1988); C. Albajar et al., Z. Phys. C44, 15 (1989).

2. C. Albajar et al., Phys. Lett. 209B, 385 (1988); J.A. Appel et al., Phys. Lett. 176B, 239 (1986).

3. P. Aurenche, R. Baier, M. Fontannez, D. Schiff, Nucl. Phys. B297, 691 (1988).

4. C. Albajar et al., Phys. Lett. B186, 217 (1987); H. Albrecht et al., Phys. Lett. B192, 245 (1987).

5. F. Abe et al., Phys. Rev. Lett. 64, 147 (1990).

6. B.L. Combridge, Nucl. Phys. B151, 429 (1979). J. Babcock, D. Sivers, and S. Wolfram, Phys. Rev. D18, 162 (1978).

7. A review of the phenomenology is presented in E.L. Berger, in Proceedings of the IX Physics in Collision Conference, Jerusalem, Israel, 1989, edited by J. Grunhaus (Editions Frontieres, Gif-sur-Yvette, France, 1990), pp. 101-118.

8. E.L. Berger, Phys. Rev. D37, 1810 (1988); E.L. Berger, in Hadrons, Quarks, and Gluons, Proceedings of the XXII Rencontre de Moriond, Les Arcs, 1987, edited by J. Tran Thanh Van (Editions Frontieres, France, 1987), pp. 3-40.

9. P. Nason, S. Dawson, and R.K. Ellis, Nucl. Phys. B303, 607 (1988).

10. W. Beenak (Mur, H. Kuijf, W.L. van Neerven, and J. Smith, Phys. Rev. D40, 54 (1989). 
11. P. Nason, S. Dawson, and R.K. Ellis, Nucl. Phys. B327, 49 (1989).

12. I. Hinchliffe, PAPAGENO 3.07 .

13. F.E. Paige and S.D. Protopopescu, Brookhaven National Laboratory Report No. 38774 (1986). The $b$ samples were generated using ISAJET version 6.21 .

14. R.K. Ellis and J. C. Sexton, Nucl. Phys, B282, 642 (1987).

15. T.D. Gottschalk, in Physics Simulations at High Energy, Madison, Wisconsin, 1986, edited by V. Barger, T.D. Gottschalk, and F. Halzen (World Scientific, Singapore 1987), p. 453.

16. A review of charm data is given in S.P.K. Tavernier, Rep. Prog. Phys. 50, 1439 (1987).

17. V. Fadin, V. Khoze, and T. Sjöstrand, Z. Phys. C48, 613 (1990); 'W. Beenakker, W.L. van Neerven, R. Meng, G.A. Schuler, and J. Smith, Nucl. Phys. B351, 507 (1991).

18. C. Albajar et al.. Phys. Lett. B256, 121 (1991).

19. "Beauty Physics at Fermilab", CDF preliminary results, presentation by J. Spalding to URA Visiting Committee at Fermilab, Jan. 19, 1991, (unpublished).

20. D. Kuebel, M. Pundurs, C.-P. Yuan, E.L. Berger, and F. E. Paige, Phys. Rev. D43, 767 (1991).

21. G. Altarelli and G. Parisi, Nucl. Phys. B126, 298 (1977).

22. W. Marciano, Phys. Rev. D29, 580 (1984).

23. E. Eichten, I. Hinchliffe, K. Lane, and C. Quigg, Rev. Mod. Phys. 56, 579 (1984).

24. M. Diemoz, F. Ferroni, E. Longo, and G. Martinelli, Z. Phys. C39, 21 (1988). 
25. E.M. Levin, M.G. Ryskin, and Yu. M. Shabelsky, Laboratoire de Physique Theorique et Hautes Energies Report No. 91/01 (1991).

26. J.C. Collins and R.K. Ellis, Fermilab Report No. 91/22-'T, (1991).

27. F. Halzen, P. Hoyer, and C.S. Kim, Phys. Lett. 195B, 74 (1987)

28. R. Meng, G.A. Schuler, J. Smith, and W.L. van Neerven, Nucl. Phys. B339, 235 (1990).

29. J.R. Cudell, O. Di Rosa, I. ten Have, A. Nisati, R. Odorico, and T. Sjöstrand, "A Comparison of Bottom Production in Different Event Generators", CERN Report CERN-TH (1991).

30. J.C. Collins, D.E. Soper, and G. Sterman, Nucl. Phys. B263, 37 (1986).

31. F.I. Olness and W.-K. Tung, Nucl. Phys. B308, 813 (1988).

32. R.M. Barnett, H.E. Haber and D.E. Soper, Nucl. Phys. B306, 697 (1988).

33. T.D. Gottschalk, Nucl. Phys. B277, 700 (1986).

34. B. R. Webber, in Proceedings of the 1990 DPF Summer Study on High Energy Physics Research Directions for the Decade, Snowmass, Colorado, June 25-July 13,1990 (to be published).

35. M. Austern, A. Barbero-Galtieri, R. Drucker, and R.P. Ely, CDF Technical Report No. CDF/ANA/ HEAVY FLAVOR/CDFR/918 (1990).

36. H.-U. Bengtsson and T. Sjöstrand, Comput. Phys. Commun. 46, 43 (1987). 

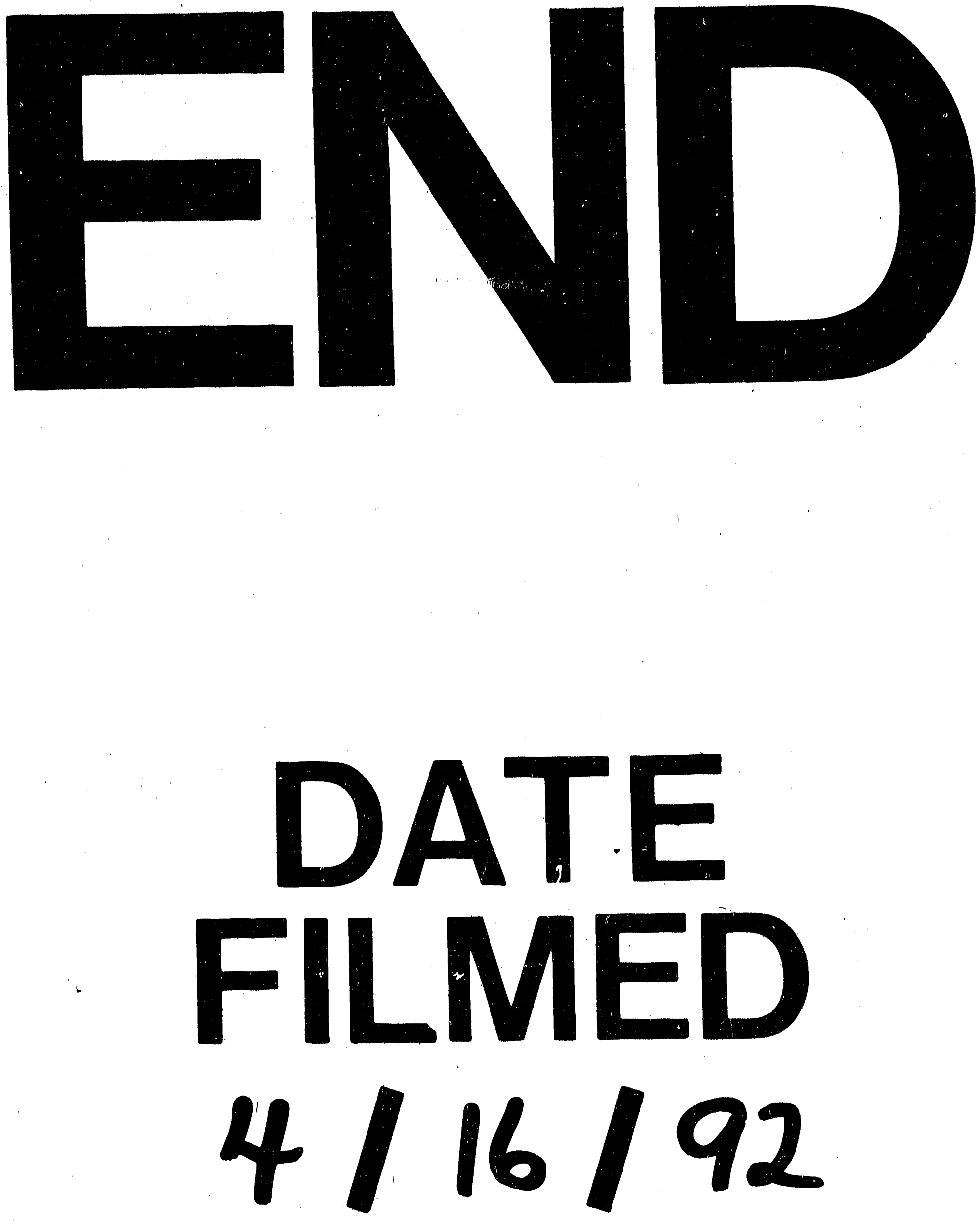

1 
